C. Mœglin

\title{
Sur la classification des séries discrètes des groupes classiques p-adiques: paramètres de Langlands et exhaustivité
}

Received November 22, 2000 / final version received February 7, 2001

Published online May 7, 2001 - (c) Springer-Verlag \& EMS 2001

\begin{abstract}
In this paper, we are giving parameters for discrete series of classical p-adic groups. We first define: the analogous of the Langlands morphism of $W_{F}$ in the $L$-group, part of the analogous of the character of the centralizer of that morphism and, to supply the missing part of the full definition of that character, the cuspidal support of the representation. Then, we state an hypothesis on the reducibility points for induced of cuspidal representations. And we prove that, under this hypothesis, the 3 data characterize discrete series.
\end{abstract}

Ici $F$ est un corps p-adique et $G(n, F)$ est un groupe classique c'est-à-dire soit un groupe spécial orthogonal sur un espace de dimension $2 n+1$, un groupe orthogonal sur un espace de dimension $2 n$, un groupe symplectique sur un espace de dimension $2 n$ ou, enfin, un groupe unitaire sur un espace vectoriel pour une extension quadratique $E$ de $F$ de dimension $n$ sur $E$. Ce groupe n'est donc pas nécessairement déployé. Les résultats de l'article ne dépende pas (apparemment) de la forme bilinéaire (symétrique, antisymétrique,...) sur l'espace vectoriel et c'est pour cela que nous ne mentionnons pas la forme dans la notation du groupe; la dépendance est cachée dans le support cuspidal partiel (dont la définition est expliquée ci-dessous). Le cas des groupes unitaires est similaire aux autres (c'est pour cela que l'on l'inclut) mais les notations sont différentes; on renvoit donc à l'appendice pour ce groupe. Le but est de décrire des paramètres des séries discrètes de $G(n, F)$ de façon à permettre une classification. La partie nouvelle est importante de ces paramètres est ce que j'appelle blocs de Jordan et caractères sur l'ensemble des blocs de Jordan; ils sont la traduction combinatoire directe de la classification de Langlands conjecturale en terme d'objets duaux. Cette traduction est expliquée en détail dans le cas le plus difficile, celui des groupes unitaires en appendice mais la compréhension de cette traduction n'est pas nécessaire, les objets étant directement à partir des représentations. Ces paramètres sont suffisamment simples pourqu'on en donne la description dès cette introduction.

Soit $\pi_{0}$ une série discrète de $G(n, F)$; on définit les blocs de Jordan de $\pi_{0}$ (dont l'ensemble est noté $\operatorname{Jor} d\left(\pi_{0}\right)$ ) de la façon suivante. Soit $\rho$ une représentation cuspidale unitaire d'un groupe linéaire, $G L\left(d_{\rho}, F\right)\left(G L\left(d_{\rho}, E\right)\right.$ dans le cas des

C. Mœglin: CNRS, case 7012, Mathematiques, 75251 Paris Cedex 05, France e-mail:moeglin@math.jussieu.fr 
groupes unitaires, où $E$ est l'extension quadratique qui définit ce groupe); ceci définit $d_{\rho}$ et soit $a \in \mathbf{N}$ (c'est-à-dire un entier non nul). On note $S D(\rho, a)$ la série discrète de $G L\left(a d_{\rho}, F\right)$ (ou $E$ comme ci-dessus) unique sous-module irréductible de l'induite:

$$
\times_{i \in[1, a]} \rho||^{(a-1) / 2-i+1} .
$$

Alors $(\rho, a)$ est dans $\operatorname{Jord}\left(\pi_{0}\right)$ par définition, si et seulement si $\rho$ est autoduale ( $\rho \simeq \rho^{*}$, sauf pour les groupes unitaires où la condition est $\rho \simeq{ }^{\sigma} \rho^{*}$ où $\sigma$ est l'élément non trivial du groupe de Galois,

l'induite pour le groupe $G\left(n+a d_{\rho}, F\right)$ :

$$
S D(\rho, a) \times \pi_{0}
$$

est irréductible

et il y a une condition de parité sur $a$ : $a$ est pair si $L(\rho, R, s)$ a un pôle en $s=0$ et impaire sinon où la représentation $R$ dépend du groupe $G(n, F)$.

Il y a une façon abstraite de définir $R$ en utilisant le groupe dual. Le cas des groupes unitaires est le plus difficile et on renvoit à l'appendice pour sa description. Dans les autres cas, le groupe dual de $G(n, F)$ est un produit direct $G^{*}(n, \mathbf{C}) \times W_{F}$ où $G^{*}(n, \mathbf{C})$ est un groupe classique complexe de rang $n$. Dans le groupe de même type $G^{*}\left(n+d_{\rho}, \mathbf{C}\right.$ de rang $n+d_{\rho}$, on considère le parabolique $P^{*}$ de Levi $G L\left(d_{\rho}, \mathbf{C}\right) \times G^{*}(n, \mathbf{C})$; le centre de $G L\left(d_{\rho}, \mathbf{C}\right)$ opère par l'application adjointe dans l'algèbre de Lie du radical unipotent de ce parabolique. Cette représentation a 2 composantes isotypiques l'une correspondant au caractère naturel et l'autre au carré de ce caractère (pour de bonnes identifications); la représentation $R$ est celle qui se réalise dans la composante isotypique correspondant au carré du caractère naturel. Précisément si $G(n, F) \simeq S p(2 n, F)$ ou est un groupe orthogonal sur un espace de dimension $2 n$, la représentation $R$ est $\wedge^{2} \mathbf{C}^{d_{\rho}}$ si $G(n, F)$ est un groupe orthogonal sur un espace de dimension $2 n+1, R \simeq S y m^{2} \mathbf{C}^{d_{\rho}}$.

Ces ensembles $\operatorname{Jord}\left(\pi_{0}\right)$ sont par construction sans multiplicité; ceci est justifié par un résultat d'Harish-Chandra, que je ne développe pas ici.

$\mathrm{Si} \pi_{0}$ est cuspidale et vérifie les hypothèses de [M1], cette définition coïncide avec celle donné en loc.cit. Les idées de Shahidi en particulier [S], convenablement généralisées et reprises en [M1], montrent que les blocs de Jordan doivent caractériser les paquets de Langlands formés de séries discrètes. On a expliqué en loc.cit que ces blocs représentent le paramètre de Langlands du paquet du moins si l'on dispose d'une forme faible des conjectures d'Arthur. Nous ne faisons pas dans cet article l'hypothèse que cette forme des conjectures d'Arthur est connue, on remplace cette hypothèse par une autre plus faible qui permet le calcul des points de réductibilité des induites de cuspidales:

$$
\rho||^{s} \times \pi_{0}
$$

où $\rho$ est autoduale comme ci-dessus, $\pi_{0}$ est, ici, cuspidale, en terme des blocs de Jordan. Cette hypothèse est simple: une telle induite est réductible en $s=s_{0}$ réel si et seulement si

$$
s_{0}= \pm\left(a_{\rho}+1\right) / 2
$$


où $a_{\rho}$ est l'élément maximal de l'ensemble $\left\{\left(a \in \mathbf{N} \mid(\rho, a) \in \operatorname{Jord}\left(\pi_{0}\right)\right\}\right.$ si cet ensemble est non vide et fini et sinon $a_{\rho}=0$ si $L(\rho, R, s)$ a un pôle en $s=0$ et $a_{\rho}=-1$ si $L(\rho, R, s)$ a un pôle en $s=0$.

Cette hypothèse entraîne la propriétés très particulière de l'ensemble $\operatorname{Jord}\left(\pi_{0}\right)$ si $\pi_{0}$ est cuspidale: soit $a \in \mathbf{N}$ avec $a \geq 3$ tel que $(\rho, a) \in \operatorname{Jord}\left(\pi_{0}\right)$ alors $(\rho, a-2) \in \operatorname{Jord}\left(\pi_{0}\right)$. Cela se trouve dans [ $\left.\mathrm{M}_{1}\right]$ pour les groupes symplectiques et orthogonaux, mais cela est réexpliqué plus directement ici dans l'appendice pour le cas des groupes unitaires qui est de loin le plus difficile (on ne reviendra donc pas la-dessus dans le corps du texte).

Avec cette hypothèse, dans cet article, on construit en plus un analogue du caractère, $\epsilon_{\pi_{0}}$ du centralisateur dans le $L$-groupe du paramètre du paquet (cf. cidessous); on utilise les blocs de Jordan au lieu d'un morphisme dans le L-groupe et une forme combinatoire du caractère qui n'est autre qu' une fonction de l'ensemble des blocs de Jordans dans \pm 1 . On ne le fait malheureusement que partiellement, car le cas cuspidal échappe en partie; en fait ce que l'on construit n'est pas le caractère mais ses "différences" c'est-à-dire:

$$
\epsilon_{\pi_{0}}(\rho, a) \epsilon_{\pi_{0}}\left(\rho, a^{\prime}\right)^{-1},
$$

pour $\rho$ fixé et $a, a^{\prime}$ tel que $(\rho, a),\left(\rho, a^{\prime}\right) \in \operatorname{Jord}\left(\pi_{0}\right)$. On accepte même $a^{\prime}=0$ si $a$ est pair en posant $\epsilon_{\pi_{0}}(\rho, 0)=1$ et on définit même $\epsilon_{\pi_{0}}(\rho, a)$ pour certains choix de $\rho$ même si $a$ est impair; on explique précisément cela à la fin de l'introduction. Pour aller plus loin, c'est-à-dire définir complètement $\epsilon_{\pi_{0}}$, il faudrait encore progresser sur les normalisations possibles des opérateurs d'entrelacement; on en parle dans le paragraphe 6 .

Pour pallier au défaut de cette construction, nous sommes obligés d'utiliser un troisième objet pour avoir vraiment des paramètres: le support cuspidal partiel. Cet objet est bien naturel mais est vraiment de nature représentation et non pas objet dual: le support cuspidal partiel, $\pi_{c u s p}$ de $\pi_{0}$ est l'unique (à isomorphisme près) représentation cuspidale irréductible d'un groupe $G\left(n^{\prime}, F\right)$ dans la même tour de Witt que $G(n, F)$ pour $n^{\prime} \leq n$ convenable tel que $\pi_{0}$ soit un sous-module d'une induite:

$$
\tau \times \pi_{\text {cusp }},
$$

où $\tau$ est n'importe quelle représentation irréductible de $G L\left(n-n^{\prime}, F\right)$ (dans le cas des groupes unitaires $G L\left(n-n^{\prime}, E\right)$ où $E$ est l'extension quadratique définissant le groupe).

Notre résultat est que sous l'hypothèse faite, les blocs de Jordan, caractère du centralisateur partiellement défini et support cuspidal sont effectivement des paramètres pour les séries discrètes:

Théorème: Sous l'hypothèse faite au début de l'introduction, l'application qui à une série discrète $\pi_{0}$ de $G(n, F)$ associe son support cuspidal, Jord $\left(\pi_{0}\right)$ et la fonction $\epsilon_{\pi_{0}}$ (définie seulement partiellement) est injective sur l'ensemble des séries discrètes. 
Au passage, on explique où l'on doit chercher la série discrète devant correspondre à un paramètre donné; toutefois on ne montre pas dans cet article qu'à tout paramètre potentiel correspond une série discrète, cela est fait dans un article ultérieur, travail commun avec Tadić.

En faisant l'hypothèse sur la réductibilité des induites de cuspidale, on calcule la mesure de Plancherel pour les cuspidales en terme de fonctions $L$; alors [M1], qui avait une hypothèse plus forte liée aux conjectures d'Arthur, montre aussi que

$$
\sum_{(\rho, a) \in \operatorname{Jord}\left(\pi_{0}\right)} a d_{\rho}=m^{*} .
$$

si $\pi_{0}$ est cuspidale, où $m^{*}$ est la dimension de la représentation naturelle de $G^{*}(n, \mathbf{C})$; la généralisation d'une telle formule aux séries discrètes (si on l'admet pour les cuspidales) résulte immédiatement du paragraphe 4 ci-dessous. Cette formule est bien naturelle quand on a en tête la classification de Langlands, mais nous n'en avons pas besoin.

On a aussi expliqué dans [M1] comment les blocs de Jordan déterminent le paramètre de Langlands (morphisme de $W_{F} \times S L(2, \mathbf{C})$ dans le $L$-groupe, conjecturalement associé aux séries discrètes, mais il faut encore des propriétés des représentations $\rho$ autoduale tel que $L(\rho, R, s)$ ait ou n'ait pas de pôle en $s=0$. Nous n'avons pas besoin de cela ici et nous ne faisons donc aucune hypothèse de ce genre. Mais cela explique pourquoi nous avons gardons la terminologie blocs de Jordan plutôt que morphisme de $W_{F} \times S L(2, \mathbf{C})$ dans le L-groupe.

Notre construction de $\operatorname{Jord}\left(\pi_{0}\right)$ est essentiellement intéressante dans le cas où $\pi_{0}$ est cuspidal; dans ce cas, notre construction est nouvelle alors qu'il est facile de remarquer que le support cuspidal usuel de $\pi_{0}$ et $\operatorname{Jord}\left(\pi_{c u s p}\right.$ détermine uniquement $\operatorname{Jord}\left(\pi_{0}\right)$. En effet, un support cuspidal pour $G(n, F)$ étant fixé (celui de $\pi_{0}$ une série discrète par exemple), on sait aisément s'il peut être le support cuspidal d'une série discrète. La condition est la suivante: le support cuspidal est la donnée d'une collection de représentations cuspidales $\rho^{\prime}||^{z^{\prime}}$ de groupes linéaires, où $\rho^{\prime}$ est unitaire et $z^{\prime} \in \mathbf{R}$ ainsi que d'une représentation cuspidale $\pi_{\text {cusp }}$ d'un groupe $G\left(n^{\prime}, F\right)$ pour $n^{\prime} \in[0, n]$. Avec les hypothèses admises sur les réductibilités on sait bien définir $\operatorname{Jord}\left(\pi_{\text {cusp }}\right.$ ) (cf. le début de l'introduction). Alors ce support cuspidal est celui d'une série discrète si et seulement si il existe un ensemble, noté Jord, de couples $(\rho, a)$, (comme au début de l'introduction), ensemble sans multiplicité tel que l'on ait l'égalité d'ensemble (ici avec multiplicité):

$$
\begin{gathered}
\left\{\rho^{\prime}||^{\left|z^{\prime}\right|}\right\} \cup\left\{\rho^{\prime}||^{\left|z^{\prime}\right|}\right\} \cup_{(\tau, b) \in \operatorname{Jord}\left(\pi_{\text {cusp }}\right)}\left\{\tau||^{|k|} ; k \in[(b-1) / 2,-(b-1) / 2]\right\} \\
=\cup_{(\rho, a) \in \operatorname{Jord}}\left\{\rho||^{|i|} ; i \in[(a-1) / 2,-(a-1) / 2]\right\} .
\end{gathered}
$$

Ce n'est pas une faute de frappe que d'avoir fait intervenir l'ensemble $\left\{\rho^{\prime}||^{\left|z^{\prime}\right|}\right\}$ avec multiplicité 2 . Remarquons que le deuxième membre de (2) est uniquement déterminé par le premier mais que l'existence de ce deuxième membre impose des propriétés fortes au support cuspidal fixé. 
Fixons donc un support cuspidal vérifiant ces propriétés; Jord est alors uniquement déterminé par le support cuspidal fixé. On dira que $\operatorname{Jord}$ contient $\operatorname{Jord}\left(\pi_{\text {cusp }}\right)$. Fixons une fonction:

$$
\epsilon: \text { Jord } \rightarrow\{ \pm 1\}
$$

pas complètement définie; on suppose que les différence (1) sont toutes définies, ainsi que $\epsilon(\rho, a)$ si $(\rho, a) \in$ Jord et $a$ est pair ou si $a$ est impair mais pour tout $a^{\prime} \in \mathbf{N},\left(\rho, a^{\prime}\right) \notin \operatorname{Jord}\left(\pi_{\text {cusp }}\right)$. C'est à ce niveau de complétion que nous définissons les fonctions $\epsilon_{\pi_{0}}$ associées à une série discrète $\pi_{0}$.

Comme le support cuspidal est fixé et que l'on a en vue de décrire toutes les séries discrètes ayant ce support cuspidal, il faut limiter les fonctions $\epsilon$ en fonction de $\pi_{\text {cusp. }}$. Ce sont des conditions qui interviennent dans la représentation de Springer et donc dans les travaux de Lusztig; je ne fais pas le lien (c'est uniquement un problème combinatoire). J'appelle $\epsilon$ admissible pour $\pi_{\text {cusp }}$ si et seulement si l'une des 2 conditions ci-dessous est satisfaites:

1 - soit il existe $\rho$ une cuspidale unitaire de $G L\left(d_{\rho}, F\right)$ et $a \neq a^{\prime} \in \mathbf{N}$ tels que $(\rho, a),\left(\rho, a^{\prime}\right) \in$ Jord avec $a>a^{\prime}$ mais il n'existe pas $\left.b \in\right] a, a^{\prime}[\operatorname{avec}(\rho, b) \in$ Jord et

$$
\epsilon(\rho, a)=\epsilon\left(\rho, a^{\prime}\right) .
$$

De plus on pose $\operatorname{Jord}^{\prime}:=\operatorname{Jord}-\left\{(\rho, a),\left(\rho, a^{\prime}\right)\right\}$ et on demande que la restriction de $\epsilon$ à $J_{o r d}$ soit admissible pour $\pi_{\text {cusp }}$ (ici c'est une définition inductive; on remplace $G(n, F)$ par le groupe $G\left(n-d_{\rho}\left(\left(a+a^{\prime}\right) / 2\right), F\right)$ et le support cuspidal fixé par le support cuspidal qui s'en déduit après avoir enlevé l'ensemble $\left(\rho,||^{|i|}\right.$ pour $i \in\left[(a-1) / 2,-\left(a^{\prime}-1\right) / 2\right]$ et on sous-entend que cette opération est possible; il $\mathrm{y}$ a déjà une petite condition)

2- pour tout $\rho$ et tout couple d'entiers $a>a^{\prime}$ tel que $(\rho, a),\left(\rho, a^{\prime}\right) \in$ Jord consécutif pour cette propriété:

$$
\epsilon(\rho, a) \neq \epsilon\left(\rho, a^{\prime}\right) \text {. }
$$

De plus, on ajoute à $\operatorname{Jord}\left(\pi_{\text {cusp }}\right)$ des couples $(\rho, 0)$ pour tout $\rho$ tel qu'il existe $a$ pair avec $(\rho, a) \in$ Jord et tel que pour $a$ minimal avec cette propriété $\epsilon(\rho, a)=1$; on demande alors encore que pour $\operatorname{Jord}\left(\pi_{\text {cusp }}\right)$ ainsi modifié et pour toute représentation cuspidale, unitaire $\rho^{\prime}$ d'un $G L\left(d_{\rho^{\prime}}, F\right)$ :

$$
\mid\left\{a^{\prime} \in \mathbf{N} \cup\{0\} ;\left(\rho^{\prime}, a^{\prime}\right) \in \operatorname{Jord}\left(\pi_{c u s p}\right\}|=|\left\{b \in \mathbf{N} ;\left(\rho^{\prime}, b\right) \in \operatorname{Jord}\right\} .\right.
$$

Dans ce papier on montre que l'application $\epsilon_{\pi_{0}}$ que je définis partiellement pour $\pi_{0}$ une série discrète dont le support cuspidal vérifie (2) ci-dessus pour Jord et $\pi_{c u s p}$ fixés, est admissible pour $\pi_{\text {cusp }}$.

Réciproquement, si Jord $\pi_{c u s p}$ et $\epsilon$ sont admissibles comme ci-dessus, on explique où il faut chercher la série discrète qui éventuellement correspond à ces données; si $\epsilon$ vérifie la $2 \mathrm{e}$ condition, la série discrète sera fortement positive (cf 1 ci-dessous) et son existence résultera d'une réciproque à 4.1. Dans le premier cas, l'assertion à démontrer est la suivante:

soit $\rho, a, a^{\prime}$ comme dans 1 ; supposons que l'on ait construit une série discrète $\pi_{0}^{\prime}$ de $G\left(n-d_{\rho}\left(a+a^{\prime}\right) / 2, F\right)$ correspondant à $J o r d^{\prime}, \pi_{c u s p}$ et $\epsilon_{\mid J o r d^{\prime}}$. On note 
$S D\left(\rho,(a-1) / 2,-\left(a^{\prime}-1\right) / 2\right)$ la série discrète non unitaire de $G L\left(d_{\rho}\left(a+a^{\prime}\right) / 2, F\right)$ unique sous module de l'induite:

$$
\times_{k \in\left[(a-1) / 2,-\left(a^{\prime}-1\right) / 2\right] \rho||^{k} .}
$$

Alors on vérifie sans difficulté que l'induite:

$$
S D\left(\rho,(a-1) / 2,-\left(a^{\prime}-1\right) / 2\right) \times \pi_{0}^{\prime}
$$

a exactement 2 sous-modules irréductibles (cf. 5.1.1 ci-dessous). Il faut démontrer que ces 2 sous-modules sont en fait des séries discrètes. Elles correspondront alors aux 2 valeurs de $\epsilon$ ayant la restriction à $\operatorname{Jord}^{\prime}$ qui correspond à $\pi_{0}^{\prime}$.

Dans la fin de cette introduction, on fixe une série discrète $\pi_{0}$ et on explique les propriétés de $\epsilon_{\pi_{0}}$. On utilisera la convention suivante: soit $b$ un entier; si l'on écrit $G(n-b, F)$ cela veut dire que l'indice de Witt de la forme (orthogonal, symplectique ou unitaire) définissant le groupe est supérieur ou égale à $b$ et le groupe écrit est le groupe correspondant à la forme de départ à laquelle on a enlevé $b$ plans hyperboliques. L'auteur reconnait que cette notation est très mauvaise pour les groupes unitaires (il faut alors lire $n-2 b$ puisque dans ce cas, $n$ est la dimension de l'espace, mais on a déjà dit que le cas des groupes unitaires est reporté à l'appendice). Donnons donc la définition de $\epsilon_{\pi_{0}}$ quand on sait le faire. Un couple $(\rho, a)$ sera toujours du type que l'on a déjà considéré: $\rho$ a la propriété d'autodualité (qui vient d'Harish-Chandra) et $a$ a la condition de parité imposée par l'existence ou non de pôle en $s=0$ de $L(\rho, R, s)$. Soit $(\rho, a) \in \operatorname{Jord}\left(\pi_{0}\right)$, on note $a_{-}$, quand il existe, le plus grand entier vérifiant $\left(\rho, a_{-}\right) \in \operatorname{Jord}\left(\pi_{0}\right)$ et $a>a_{-}$. Alors, on construit $\epsilon_{\pi_{0}}$ en imposant les propriétés:

(P1) si $(\rho, a)$ est comme ci-dessus avec $a_{-}$bien défini, alors:

$$
\epsilon_{\pi_{0}}(\rho, a)=\epsilon_{\pi_{0}}\left(\rho, a_{-}\right) \Leftrightarrow
$$

il existe une représentation $\pi^{\prime}$ de $G\left(n-d_{\rho}\left(a-a_{-}\right) / 2, F\right)$ et une inclusion:

$$
\pi_{0} \hookrightarrow \times_{k \in\left[1,\left(a-a_{-}\right) / 2\right]} \rho||^{(a-1) / 2-k+1} \times \pi^{\prime} .
$$

(P2) si $(\rho, a) \in \operatorname{Jord}\left(\pi_{0}\right)$ et $a$ est pair avec $a$ minimum avec cette propriété, on a la même propriété que ci-dessus mais en posant $a_{-}=0$ et $\epsilon_{\pi_{0}}\left(\rho, a_{-}\right)=1$.

On reprend la notation $\pi_{\text {cusp }}$ qui vient du support cuspidal de $\pi_{0}$; on veut encore définir $\epsilon_{\pi_{0}}(\rho, a)$ pour $a$ impair si pour tout $a^{\prime} \in \mathbf{N},\left(\rho, a^{\prime}\right) \notin \operatorname{Jord}\left(\pi_{\text {cusp }}\right)$. On n'a pas de façon canonique de le faire mais on fixe une normalisation des opérateurs d'entrelacement vérifiant les conditions de [A]; on note $N(\cdots, s)$ ces opérateurs d'entrelacement normalisés quand ils ne dépendent que d'une variable $s \in \mathbf{C}$. Soit donc $\rho$ avec la propriété ci-dessus et $(\rho, a) \in \operatorname{Jord}\left(\pi_{0}\right)$. On note $\operatorname{SD}(\rho, a)$ la série discrète de $G L\left(d_{\rho} a, F\right)$ notée ci-dessus $S D(\rho,(a-1) / 2,-(a-1) / 2)$. On fixe $\epsilon_{\pi_{0}}(\rho, a)$ comme étant le scalaire parlequel opère l'opérateur d'entrelacement normalisé en $s=0$ :

$$
N\left(S D(\rho, a) \times \pi_{0}, s\right): S D(\rho, a)||^{s} \times \pi_{0} \rightarrow S D(\rho, a)||^{-s} \times \pi_{0} .
$$


On a à vérifier et cela est fait en 6.1, que cette définition vérifie bien (P1) ci-dessus. Si $\pi_{\text {cusp }}$ n'a pas la propriété imposée une telle définition est toujours possible. Elle ne vérifie (P1) que pour un choix particulier de normalisation (cf. 6.1 .3 cidessous); ce choix n'est quand même bien défini qu'à une fonction holomorphe inversible près ce qui ne permet quand même pas de définir canoniquement le scalaire cherché; il est vraisemblable que c'est quand même en travaillant sur la normalisation des opérateurs d'entrelacement que l'on obtiendrait une définition complète de $\epsilon_{\pi_{0}}$.

Cela termine ce que je sais faire pour construire $\epsilon_{\pi_{0}}$; mais comme le dit le théorème cela suffit du moment que l'on traîne $\pi_{c u s p}$ dans les énoncés. Dans le dernier paragraphe, j'explique ce que je pense être vrai pour les cuspidales; j'ai comme exemple les représentations cuspidales quadratiques unipotentes de [M2]. C'est cet exemple que je généralise, c'est peut-être un peu abusif. Si on sait définir complètement $\epsilon_{\pi}$ pour les cuspidales, je montre que l'on sait alors définir complètement $\epsilon_{\pi_{0}}$ pour les séries discrètes. On vérifie aisément que si l'application

$$
\pi \mapsto \operatorname{Jord}(\pi), \epsilon_{\pi}
$$

est injective sur l'ensemble des cuspidales pour tout $G\left(n^{\prime}, F\right)$ avec $n^{\prime} \leq n$, il en est de même quand on étend cette application aux séries discrètes.

Et la description de l'image doit être simple: on fixe un ensemble Jord de couple $(\rho, a)$, la parité de $a$ étant imposé par l'existence ou non de pôle pour la fonction $L(\rho, R, s)$ en $s=0$ et tel que:

$$
\sum_{(\rho, a) \in \text { Jord }} a d_{\rho}=m^{*}
$$

où $m^{*}$ est défini ci-dessus. Soit

$$
\epsilon: \text { Jord } \rightarrow\{ \pm 1\}
$$

vérifiant

$$
\times_{(\rho, a) \in \operatorname{Jord}} \epsilon(\rho, a)^{a d_{\rho}}
$$

est une constante fixée (c'est le calcul de la restriction du caractère au centre soit de $\operatorname{Sp}(2 n, \mathbf{C})$ soit de $O(2 n+1, \mathbf{C})$, qui représente les $L$-groupes $)$. Alors il existe une série discrète $\pi_{0}$ vérifiant $\operatorname{Jord}\left(\pi_{0}\right)=\operatorname{Jord}$ et $\epsilon_{\pi_{0}}=\epsilon$.

Grâce aux résultats de Shahidi pour les représentations cuspidales ayant un modèle de Whittaker, nos résultats sont sans hypothèses pour les séries discrètes ayant des modèles de Whittaker; mais ce cas n'est pas vraiment nouveau quand on interprète avec nos définition les articles de G. Muić [Mu1] et [Mu 2]. Nos résultats sont aussi sans hypothèses pour les séries discrètes quadratiques unipotentes des groupes orthogonaux ou symplectiques: c'est-à-dire pour les séries discrètes telles que le support cuspidal est constitué de caractères quadratiques et d'une représentation cuspidale quadratique unipotente au sens de [M2]. Ce dernier cas doit correspondre aux séries discrètes, $\pi_{0}$, tel que $\operatorname{Jord}\left(\pi_{0}\right)$ ne contient que des 
couples $(\rho, a)$ où $\rho$ est un caractère quadratique; la correspondance n'est pas totalement établie car il reste à montrer que les représentations $\pi_{0}$ cuspidales avec la propriété que si une induite du type:

$$
\rho||^{s} \times \pi_{0}
$$

est réductible pour $s=s_{0}$ (réel) différent de 0 ou $\pm 1 / 2$ alors $\rho$ est un caractère quadratique, sont nécessairement des représentations cuspidales quadratiques unipotentes au sens de [M2].

Pour terminer cette introduction, il me reste à remercier J.-L. Walspurger qui m'a expliqué à de nombreuses reprises les travaux d'Harish-Chandra et a en particulier complété $[\mathrm{W}]$ pour y inclure un résultat dont j' ai besoin de façon déterminante: l'opérateur d'entrelacement standard associé à une induite d'un parabolique maximal à partir d'une série discrète du Levi est d'ordre 0 ou -1 en tout point de l'axe unitaire. Il obtient ce résultat comme corollaire immédiat (dit-il) des travaux d'Harish-Chandra. Ce résultat est clé pour savoir que les ensembles $\operatorname{Jord}\left(\pi_{0}\right)$, pour $\pi_{0}$ une série discrète sont sans multiplicité.

Jantzen dans $[\mathrm{J}]$ a aussi abordé le problème de l'exhaustivité.

Dans la partie principale de l'article, on suppose que $G(n, F)$ est soit le groupe $S p(2 n, F)$ soit le groupe d'une forme orthogonale de dimension $2 n$ ou $2 n+1$; pour le cas du groupe orthogonal d'un espace de dimension paire, on a besoin de l'appendice au paragraphe 4 pour déduire des calculs de mesure de Plancherel de notre hypothèse sur les points de réductibilité. Dans l'appendice, on traite le cas des groupes unitaires, plus exactement on explique les paramètres pour ces groupes et on montre que pour obtenir les résultats pour ces groupes, il faut appliquer les démonstrations ci-dessous en remplaçant partout les groupes linéaires $G L(\cdots, F)$ par $G L(\cdots, E)$ où $E$ est l'extension quadratique de $F$ qui définit le groupe unitaire. Le lecteur peut lire tout le texte en utilisant une notation intermédiaire en posant $F^{\prime}=F$ sauf si $G$ est un groupe unitaire où $F^{\prime}=E$; c'est ce que l'on a plus ou moins fait dans l'introduction, mais je pense qu'il est plus clair pour le lecteur de séparer nettement ces 2 cas.

\section{Notations}

On notera || la valeur absolue du déterminant pour un groupe linéaire précisé par le contexte.

On a déjà introduit les 2 notations ci-dessous mais on les rappelle car elles seront constantes: soit $\rho$ une représentation cuspidale irréductible d'un groupe linéaire $G L\left(d_{\rho}, F\right)$ (cela définit $\left.d_{\rho}\right)$ et soit $a \in \mathbf{N}$. On note $S D(\rho, a)$ la représentation de Steinberg généralisée grâce à $\rho$ du groupe $G L\left(a d_{\rho}, F\right)$. Soit $x, y$ des réels tels que $x-y+\in \mathbf{N}$. On note $S D(\rho, x, y)$ l'unique sous-module irréductible de l'induite:

$$
\times_{i \in[1, x-y+1]} \rho||^{x-i+1} \text {. }
$$

On a l'isomorphisme:

$$
S D(\rho, x, y) \simeq S D(\rho, x-y+1)||^{(x+y) / 2} .
$$




\section{Définition des séries discrètes fortement positives}

Définissons les séries discrètes, $\pi_{0}$, fortement positives comme étant les représentations irréductibles telles que quelque soit la collection de couples $\left(\rho_{z}, x_{z}\right)$, où $\rho_{z}$ est une représentation cuspidale unitaire d'un groupe linéaire $G L\left(d_{\rho_{z}}, F\right)$ et où $x_{z} \in \mathbf{R}$ ( $z$ parcourt un ensemble d'indices) telle qu'il existe une représentation cupidale de $G\left(n-\sum_{\left(\rho_{z}, x_{z}\right)} d_{\rho_{z}}, F\right)$, $\pi_{\text {cusp }}$ et une inclusion:

$$
\pi_{0} \hookrightarrow \times{ }_{\left(\rho_{z}, x_{z}\right)} \rho||^{x_{z}} \times \pi_{c u s p}
$$

alors $x_{z}>0$ pour tout $\left(\rho_{z}, x_{z}\right)$ intervenant. Le fait qu'une représentation ayant cette propriété est une série discrète résulte du critère de Casselman. Mais ce sont des séries discrètes très particulières.

\section{Deux notations}

Soit $\rho$ une représentation cuspidale unitaire d'un groupe $G L\left(d_{\rho}, F\right)$ et soient $x, y \in \mathbf{R}$ tels que $x-y+1 \in \mathbf{N}$. On note:

$$
S D(\rho, x, y)
$$

la série discrète non unitaire (en général) de $G L\left(d_{\rho}(x-y+1), F\right)$ unique sousmodule de l'induite:

$$
\mathrm{X}_{i \in[1, x-y+1]} \rho||^{x-i+1} .
$$

Si $a$ est un entier, on pose:

$$
S D(\rho, a)=S D(\rho,(a-1) / 2,-(a-1) / 2) .
$$

C'est une série discrète unitaire de $G L\left(d_{\rho} a, F\right)$.

\section{Quelques propriétés élémentaires des séries discrètes}

3.1 Lemme: Soit $\pi_{0}$ une série discrète qui n'est pas fortement positive, alors il existe une représentation cuspidale unitaire $\rho$ d'un groupe linéaire $G L\left(d_{\rho}, F\right)$ et des réels $x$, $y$ vérifiant $x-y+1 \in \mathbf{N}$ et $y \leq 0$ ainsi qu'une série discrète irréductible $\pi_{0}^{\prime}$ de $G\left(n-d_{\rho}(x-y+1), F\right)$ tel que:

$$
\pi_{0} \hookrightarrow S D(\rho, x, y) \times \pi_{0}^{\prime} .
$$

Fixons un ensemble d'indices totalement ordonné, $Z$ (pour faciliter l'écriture, on suppose que $Z$ est un intervalle de $\mathbf{N}$ et que l'ordre est induit par celui de $\mathbf{N}$ ); et pour tout $z \in Z$ fixons un couple $\left(\rho_{z}, x_{z}\right)$ de couples où $\rho_{z}$ est une représentation cuspidale unitaire de $G L\left(d_{\rho_{z}}, F\right)$ et où $x_{z} \in \mathbf{R}$; fixons aussiune représentation cuspidale $\pi_{\text {cusp }}$ de $G\left(n-\sum_{z \in Z} d_{\rho_{z}}, F\right)$ (on suppose $\sum_{z \in Z} d_{\rho_{z}}$ est inférieur à l'indice de Witt de la forme définissant $G(n, F)$ ). Supposons, comme cela est possible pour de bons choix que:

$$
\pi_{0} \hookrightarrow \times_{z \in Z} \rho_{z}||^{x_{z}} \times \pi_{\text {cusp }} .
$$


L'ordre dans lequel interviennent les représentations $\rho_{z}||^{x_{z}}$ est celui imposé par l'ordre de $Z$ au sens suivant: si $z>z^{\prime}$ dans $Z$, alors, en (1), $\rho_{z}||^{x_{z}}$ est plus à gauche que $\rho_{z^{\prime}}||^{x} z^{\prime}$.

Partant d'une telle inclusion, on peut en déduire d'autres en utilisant les propriétés d'irréductibilité pour les induites des groupes linéaires. Fixons $z \in Z$ et posons $\rho=\rho_{z}$. Notons $t$ un entier tel que $z+t-1 \in Z$ et

$\rho_{z+i-1} \simeq \rho$,

$x_{z+i-1}=x_{z}+i-1$ pour tout $i \in[1, t]$,

et aussi que (1) se factorise par une inclusion:

(2) $\pi_{0} \hookrightarrow \times_{z^{\prime} \in Z ; z^{\prime}>z+t-1} \rho_{z^{\prime}}^{\prime}\left\|^{x_{z^{\prime}}} \times S D(\rho, z+t-1, z) \times{ }_{z^{\prime} \in Z ; z^{\prime}<z} \rho_{z^{\prime}}^{\prime}\right\|^{x_{z^{\prime}}}$ $\times \pi_{\text {cusp. }}$. Quitte à prendre $t=1$, on aura toujours une telle situation sauf dans le cas cuspidal. Supposons que $z+t \in Z$, c'est-à-dire que $z+t-1$ n'est pas le plus grand élément de $Z$. Montrons que l'on est dans l'un des 3 cas ci-dessous:

$\rho_{z+t+1} \simeq \rho$ et $x_{z+t+1}=x_{z}-1$, cas où l'on ne peut améliorer la situation et qu'il faudra donc éviter (par des hypothèses de minimalité sur $x_{z}$ )

ou $\left(\rho_{z+t+1}, x_{z+t+1}\right)=\left(\rho, x_{z}+t\right)$ et on peut améliorer l'inclusion (2) ci-dessus en remplaçant $t$ par $t+1$,

ou on peut changer l'ordre de $Z$ de façon à faire passer $\left(\rho_{z+t}, x_{z+t}\right)$ au-dessus de tous les couples $\left(\rho, x_{z+i-1}(i \in[1, t]\right.$ tout en gardant un analogue de (2) avec $S D\left(\rho, x_{z}+t-1, x_{z}\right)$ sans changement; c'est à dire que l'on change l'ordre dans l'ensemble d'indice $Z$ de façon à transformer $z+i-1$ en $z+i$, pour tout $i \in[1, t]$ alors que $z+t$ lui deviendra $z$.

Cela résulte directement de la propriété d'irréductibilité pour des groupes linéaires:

$$
\rho_{0}||^{x_{0}} \times S D\left(\rho, x_{z}+t-1, x_{z}\right)
$$

est irréductible si et seulement si $\left(\rho_{0}, x_{0}\right)$ n'est pas l'un des couples $\left(\rho, x_{z}-1\right)$ ou $\left(\rho, x_{z}+t\right)$. On l'applique à $\rho_{0}=\rho_{z+t}$ et $x_{0}=x_{z+t}$. Si l'on a irréductibilité, on peut commuter et l'on est dans le 3 e cas. Si l'on a réductibilité, on distingue, quand $x_{z+t}=x_{z}-1$ et $\rho_{z+t} \simeq \rho$ on est dans le premier cas ci-dessus. Il reste le cas où $\rho_{z+t} \simeq \rho$ et $x_{z+t}=x_{z}+t$. Dans ce dernier cas, Zelevinski ajoute que l'induite cidessus est de longueur exactement 2 ayant comme sous-module $S D\left(\rho, x_{z}+t, x_{z}\right)$ et comme quotient un sous-module de la représentation induite:

$$
S D\left(\rho, x_{z}+t-1, x_{z}\right) \times \rho||^{x_{z}+t} .
$$

On est donc soit dans le cas 2 soit dans le cas 3 suivant que (1) se factorise par le sous-module ou par le quotient.

Le lemme se démontre alors ainsi: fixons $Z, \pi_{\text {cusp }}$ tel que (1) soit réalisé. Fixons aussi $\rho$ une représentation cuspidale unitaire d'un groupe $G L\left(d_{\rho}, F\right)$. Fixons alors $z \in Z$ tel que $x_{z}$ soit minimal parmi les réels de l'ensemble $\left\{x_{z^{\prime}}\right\}$ pour $z^{\prime} \in Z$ tel que $\rho_{z^{\prime}} \simeq \rho$. Eventuellement plusieurs choix sont possibles avec cette propriété; on impose alors à $z$ d'être maximum avec ces propriétés. En utilisant la description des cas ci-dessus et la minimalité de $x_{z}$ (pour $\rho$ fixé), on en déduit qu'il existe $t \in \mathbf{N}$ 
tel que $z+t-1$ soit le plus grand élément de $Z$, quitte à avoir changé l'ordre, ce qui ne fait qu'augmenter le $z$ de départ) et une représentation $\pi^{\prime}$ de $G L\left(d_{\rho} t, F\right)$ tel que:

$$
\pi_{0} \hookrightarrow S D\left(\rho, x_{z}+t-1, x_{z}\right) \times \pi^{\prime} .
$$

Puisque $\pi_{0}$ est une série discrète qui n'est pas fortement positive $x_{z} \leq 0$ (au moins pour $\rho$ bien choisi) et on pose:

$$
x_{z}+t-1=x ; x_{z}=y
$$

et on a $\sum_{i \in[x, y]} k>0$ car $\pi_{0}$ est une série discrète. On va démontrer que $\pi^{\prime}$ est nécessairement une série discrète.

On fait pour $\pi^{\prime}$ ce que l'on a fait pour $\pi_{0}$; on a un analogue $Z^{\prime}$ de $Z$ tel que l'analogue de (1) soit vérifié pour $\pi^{\prime}$ (on doit même garder la même représentation $\pi_{\text {cusp }}$ ). Si $\pi^{\prime}$ n'est pas une série discrète, pour un bon choix d'ordre sur $Z^{\prime}$, il existe $z_{1} \in Z^{\prime}$ tel que:

$$
\sum_{z^{\prime} \in Z^{\prime} ; z^{\prime} \geq z_{1}} x_{z^{\prime}} \leq 0
$$

Fixons $Z^{\prime}$ et $z_{1} \in Z^{\prime}$ de tel sorte que ceci soit vérifié. En travaillant comme pour $\pi_{0}$ (en fixant $x_{z}$ minimal parmi les $x_{z^{\prime}}$ pour $z^{\prime} \geq z_{1}$ ) on montre que l'on peut l'on peut trouver un ensemble de triplet $\left(\tau_{i}, \ell_{i}, m_{i}\right)$ pour $i$ dans un ensemble d'indice totalement ordonné $I$ (encore identifié à un intervalle de $\mathbf{N}$ ), où pour tout $i \in I, \tau_{i}$ une représentation cuspidale unitaire de $G L\left(d_{\tau}, F\right), \ell_{i}, m_{i} \in \mathbf{R}$ avec $\ell_{i}-m_{i}+1 \in \mathbf{N}$ avec les 2 propriétés suivantes:

on a l'égalité d'ensemble avec multiplicités mais non ordonnés

$$
\cup_{i \in I, j \in\left[\ell_{i}, m_{i}\right]}\left(\tau_{i}, j\right)=\cup_{z^{\prime} \in Z^{\prime} ; z^{\prime} \geq z_{1}}\left(\rho_{z^{\prime}}, x_{z^{\prime}}\right)
$$

et l'inclusion:

$$
\pi^{\prime} \hookrightarrow \times_{i \in I} S D\left(\tau_{i}, \ell_{i}, m_{i}\right) \times_{z^{\prime} \in Z^{\prime} ; z^{\prime}<z_{1}} \rho_{z^{\prime}}||^{x_{z^{\prime}}} \times \pi_{c u s p} ;
$$

On continue de prendre l'ordre décroissant de I quand on va de gauche à droite dans l'écriture. Et par la construction, on impose aussi:

$$
m_{i}<m_{i-1}
$$

si $i, i-1 \in I$.

De la première propriété et du choix de $z_{1}$ résultent:

$$
\sum_{i} \sum_{k \in\left[\ell_{i}, m_{i}\right]} k \leq 0
$$

Soit $i^{\prime}$ le plus grand élément de $I$ tel que:

$$
\sum_{k \in\left[\ell_{i^{\prime}}, m_{i^{\prime}}\right]} k \leq 0 .
$$

Si $i^{\prime}$ n'est pas le plus grand élément de $I$, l'induite $S D\left(\tau_{j}, \ell_{j}, m_{j}\right) \times S D\left(\tau_{i^{\prime}}, \ell_{i^{\prime}}, m_{i^{\prime}}\right)$ (d'un groupe linéaire convenable) est irréductible pour $j \in I$ avec $j>i^{\prime}$ : en 
effet $m_{j} \leq m_{i^{\prime}}$ et donc nécessairement $\ell_{j}>\ell_{i^{\prime}}$. On est alors dans le cas des segments inclus l'un dans l'autre de Zelevinski. On a donc montré l'existence d'une représentation $\pi^{\prime \prime \prime}$ tel que avec le $i^{\prime}$ choisi ci-dessus:

$$
\pi_{0} \hookrightarrow S D(\rho, x, y) \times S D\left(\tau_{i^{\prime}}, \ell_{i^{\prime}}, m_{i^{\prime}}\right) \times \pi^{\prime \prime \prime} .
$$

Pour avoir une contradiction, il suffit de vérifier que, pour $\rho, x, y$ définis au début de la démonstration, l'induite

$$
S D(\rho, x, y) \times S D\left(\tau_{i^{\prime}}, \ell_{i^{\prime}}, m_{i^{\prime}}\right)
$$

du groupe linéaire correspondant est irréductible, puisqu'alors, il existerait une représentation $\pi^{\prime \prime \prime \prime}$ tel que:

$$
\pi_{0} \hookrightarrow S D\left(\tau_{i^{\prime}}, \ell_{i^{\prime}}, m_{i^{\prime}}\right) \times \pi^{\prime \prime \prime \prime},
$$

avec $\sum_{k \in\left[\ell_{i^{\prime}}, m_{i^{\prime}}\right]} k \leq 0$. Pour avoir l'irréductibilité, il suffit que $\tau_{i^{\prime}}$ ne soit pas isomorphe à $\rho$ et si ce n'est pas vrai, avec la minimalité de $y$, on a sûrement:

$$
y \leq m_{i^{\prime}} .
$$

On a donc aussi $x \geq \ell_{i^{\prime}}$ et le premier segment contient le second. Cela termine la preuve.

Avec des méthodes analogues on démontre la remarque suivante:

3.2 Remarque: Soit $\pi_{0}$ une série discrète et soit $(\rho, x, y)$ un triplet formé d'une représentation cuspidale unitaire $\rho$ de $G L\left(d_{\rho}, F\right)$ et de deux réels $(x, y)$ tels que $x-y+1 \in \mathbf{N}$. On suppose qu'il existe une représentation $\pi^{\prime}$ de $G\left(n-d_{\rho}(x-\right.$ $y+1), F)$ tel que

$$
\pi_{0} \hookrightarrow S D(\rho, x, y) \times \pi^{\prime}
$$

et que $y$ soit minimal avec cette propriété $x$ et $\rho$ étant fixés. Alors $\pi^{\prime}$ est une série discrète.

Comme on utilisera cette remarque, il vaut mieux indiquer la preuve même si elle est élémentaire. On raisonne comme ci-dessus pour $\pi^{\prime}$ et on montre que si $\pi^{\prime}$ n'est pas une série discrète, il existe $(\tau, \ell, m)$ tel que $\tau$ soit une cuspidale unitaire de $G L\left(d_{\tau}, F\right)$ et $\ell, m$ sont des réels tels que $\ell-m+1 \in \mathbf{N}$ et une représentation irréductible $\pi^{\prime}$ de $G\left(n-d^{\prime}, F\right)$ pour $d^{\prime}$ convenable tels que:

$$
\pi_{0} \hookrightarrow S D(\rho, x, y) \times S D(\tau, \ell, m) \times \pi^{\prime \prime}
$$

et $\sum_{k \in[\ell, m]} k \leq 0$. On ne peut conclure comme ci-dessus si $m<y$, ce que nous supposons donc. Dans ce cas, l'induite:

$$
S D(\rho, x, y) \times S D(\tau, \ell, m)
$$

n'est pas irréductible seulement si $\tau \simeq \rho$ et $\ell \in] x, y-1]$. Si cela se produit l'induite est de longueur exactement 2 avec un unique sous-module $S D(\rho, x, m) \times$ $S D(\rho, \ell, y)$ (ce dernier facteur disparaissant si $\ell=y-1$ ). La minimalité de $y$ dans l'énoncé assure que dans (3) on ne peut pas remplacer l'induite par ce sous-module et que l'on peut donc la remplacer par le quotient. D'où:

$$
\pi_{0} \hookrightarrow S D(\tau, \ell, m) \times S D(\rho, x, y) \times \pi^{\prime \prime} .
$$

Ce qui est la contradiction cherchée. 


\section{Propriété des blocs de Jordan}

Les blocs de Jordan ont été définis dans l'introduction; rappelons-en les grandes lignes. Pour que $(\rho, a)$ soit un bloc de Jordan d'une série discrète $\pi_{0}$, il faut que $\rho$ soit une représentation cuspidale unitaire irréductible d'un $G L\left(d_{\rho}, F\right)$ (ce qui définit $d_{\rho}$ ), autoduale et $a$ à une condition de parité qui dépend de $\rho$ (cf. l'introduction). Puis $(\rho, a)$ est effectivement un bloc de Jordan de $\pi_{0}$ si et seulement si l'induite:

$$
S D(\rho,(a-1) / 2,-(a-1) / 2) \times \pi_{0}
$$

est irréductible. L'hypothèse de base de tout l'article entraîne que si $\pi_{0}$ est cuspidale, on a la description précise (?) des blocs de Jordan: soit $\rho$ comme ci-dessus et noton $x_{\rho}$ le réel positif ou nul tel que l'induite $\left.\rho\right|^{x_{\rho}} \times \pi_{0}$ est réductible, alors $x_{\rho}$ est un demi-entier et $(\rho, a) \in \operatorname{Jord}\left(\pi_{0}\right)$ si et seulement si $x_{\rho} \geq 1, a$ a la parité de $2 x_{\rho}-1$ et $a \leq 2 x_{\rho}-1$. Ce n'est pas comme cela que l'on a exprimé l'hypothèse de base dans l'introduction mais la formulation est équivalente (cf. l'appendice pour le cas le plus difficile qui est celui des groupes unitaires). Le problème est maintenant de donner des propriétés de $\operatorname{Jord}\left(\pi_{0}\right)$ en supposant seulement $\pi_{0}$ une série discrète. Ceci sera fait en 4.1 et 4.2 , ci-dessous.

La proposition 4.1 ci-dessous est une des propositions clés de l'article puiqu'elle règle le cas des séries discrètes fortement positives: ces séries discrètes sont uniquement caractérisées par l'ensemble de leur bloc de Jordan et leur support cuspidal partiel (encore que celui-ci ne joue qu'un rôle mineur); cela sera expliquée au paragraphe 5. C'est aussi une des seules propositions dont la démonstration est un peu sophistiquée, c'est-à-dire utilisant autre chose que des calculs de modules de Jacquet; il en sera de même de 4.2 ci-dessous.

4.1 Proposition: Soit $\pi_{0}$ une série discrète fortement positive; alors il existe une représentation cuspidale $\pi_{\text {cusp }}$ et pour tout $(\rho, a) \in \operatorname{Jord}\left(\pi_{\text {cusp }}\right)$ (en acceptant pour $\rho$ fixé tel que $L(\rho, R, s)$ a un pôle en $s=0, a=0$ au plus une fois) il existe un entier $t_{(\rho, a)}$ éventuellement nul tel que si $(\rho, a)$ et $\left(\rho, a^{\prime}\right)$ sont dans Jord $\left(\pi_{\text {cusp }}\right)$ avec $a<a^{\prime}$ alors:

$$
(a-1) / 2+t_{(\rho, a)}<\left(a^{\prime}-1\right) / 2+t_{\left(\rho^{\prime}, a^{\prime}\right)}
$$

et $\pi_{0}$ est l'unique sous-module de l'induite:

$$
\times_{(\rho, a) \in \operatorname{Jord}\left(\pi_{\text {cusp }}\right)} S D\left(\rho,(a-1) / 2+t_{(\rho, a)},(a+1) / 2\right) \times \pi_{\text {cusp }},
$$

où les couples $(\rho, a)$ sont ordonnés de tel sorte que $(\rho, a)$ arrive avant (c'est-à-dire à gauche dans l'écriture ci-dessus) ( $\left.\rho, a^{\prime}\right)$ si $a<a^{\prime}$.

Pour la suite, on a besoin d'être plus clair sur la possibilité d'ajouter $(\rho, 0)$ à $\operatorname{Jord}\left(\pi_{\text {cusp }}\right)$; l'énoncé dit que l'on peut le faire une fois si $L(\rho, R, s)$ a un pôle en $s=0$ et la conclusion dit qu'on le fait exactement pour tous les $\rho$ tel que $L(\rho, R, s)$ a un pôle en $s=0$ si il existe un entier $t_{\rho, 0}$ et une représentation $\sigma$ de $G\left(n-d_{\rho} t_{\rho, 0}, F\right)$ avec une inclusion:

$$
\pi_{0} \hookrightarrow S D\left(\rho, t_{\rho, 0}-1 / 2,1 / 2\right) \times \sigma .
$$


En effet si une telle inclusion est vraie, un bête calcul de module de Jacquet impose la présence de $(\rho, a) \in \operatorname{Jord}\left(\pi_{\text {cusp }}\right)$ tel que $(a+1) / 2=1 / 2$ ce qui entraîne $a=0$. Et la réciproque est claire grâce aux propriétés d'ordre.

La démonstration déjà donnée dans les lemmes précédents, montre qu'il existe un ensemble de triplets $(\tau, x, y)$ et une représentation cuspidale $\pi_{c u s p}$ tel que:

$$
\pi_{0} \hookrightarrow \times_{(\tau, x, y)} S D(\tau, x, y) \times \pi_{c u s p},
$$

où on ordonne les triplets tels que $(\tau, x, y)$ arrive avant $\left(\tau, x^{\prime}, y^{\prime}\right)$ si $y \leq y^{\prime}$. La propriété de positivité forte assure que $y>0$ pour tous les triplets intervenant.

On montre d'abord que tous les $y$ intervenant ci-dessus sont des $1 / 2$ entiers et que les $\tau$ intervenant sont autoduales. S'il n'en est pas ainsi, on fixe $\left(\tau_{0}, x_{0}, y_{0}\right)$ maximal sur l'ordre mis dans l'écriture de (1) tel que $y_{0}$ ne soit par un demi-entier ou tel que $\tau_{0}$ ne soit pas autoduale. On vérifie sans problème que:

$$
\pi_{0} \hookrightarrow \times_{(\tau, x, y) \neq\left(\tau_{0}, x_{0}, y_{0}\right)} S D(\tau, x, y) \times S D\left(\tau_{0}, x_{0}, y_{0}\right) \times \pi_{\text {cusp }} .
$$

En outre

$$
S D\left(\tau_{0}, x_{0}, y_{0}\right) \times \pi_{c u s p} \hookrightarrow S D\left(\tau_{0}, x_{0}, y_{0}+1\right) \times\left.\tau_{0}\right|^{y_{0}} \times \pi_{c u s p} .
$$

Comme

$$
\tau_{0}||^{y_{0}} \times \pi_{\text {cusp }}
$$

est irréductible puisque $y_{0}$ n'est pas un demi-entier ou $\tau_{0}$ n'est pas autoduale, on peut transformer (1) en:

$$
\pi_{0} \hookrightarrow \times{ }_{(\tau, x, y) \neq\left(\tau_{0}, x_{0}, y_{0}\right)} S D(\tau, x, y) \times S D\left(\tau_{0}, x_{0}, y_{0}+1\right) \times \tau_{0}^{*}||^{-y_{0}} \times \pi_{c u s p} .
$$

Ceci contredit le fait que $\pi_{0}$ est fortement positive.

On va maintenant utiliser les opérateurs d'entrelacement standard pour calculer ces triplets en fonction de $\operatorname{Jord}\left(\pi_{c u s p}\right)$. On fixe $(\rho, a)$ en supposant $\rho$ autodual et on regarde les induites:

$$
S D(\rho, a)||^{s} \times \pi_{0}
$$

et les opérateurs d'entrelacement standard:

$$
S D(\rho, a)||^{s} \times \pi_{0} \rightarrow S D(\rho, a)||^{-s} \times \pi_{0}
$$

au voisinage de 0 que l'on note $M\left(\rho, a, \pi_{0}, s\right)$. D'après Harisch-Chandra et Waldspurger [W] on sait que le composé:

$$
M\left(\rho, a, \pi_{0},-s\right) M\left(\rho, a, \pi_{0}, s\right)
$$

est une fonction $d\left(\rho, a, \pi_{0}, s\right)$ holomorphe en $s=0$ exactement quand l'induite est réductible et quand l'induite est irréductible le pôle est d'ordre 2 exactement. Cette fonction $d(s)$ se calcule aisément en fonction des données intervenant dans (1) à condition de savoir calculer son analogue quand $\pi_{0}$ est remplacé par $\pi_{c u s p}$. En admettant les points d'irréductibilité des cuspidales on a aussi admis le calcul de $d\left(\rho, a, \pi_{c u s p}, s\right)$ à une fonction holomorphe inversible près (ceci est expliqué en 
détails dans le cas des groupes unitaires dans l'appendice). On le connait aussi pour les groupes linéaires et $d\left(\rho, a, \pi_{0}, s\right)$ est le produit évident sur toutes les données parce que cette fonction ne dépend que du support cuspidal. Il y a alors différentes façons de faire les calculs car il y a des simplifications. Pour simplifier, on met en place une récurrence.

On fixe $\rho$ et on ordonne les triplets $(\tau, x, y)$ intervenant dans (1) de tel sorte que $\left(\tau, x^{\prime}, y^{\prime}\right)$ arrive avant $(\rho, x, y)$ si $\tau \not \varnothing \rho$ ou si $\tau \simeq \rho$ mais $y^{\prime}<y$. On récrit l'ensemble des $(\rho, x, y)$ intervenant dans (1) sous la forme $\left(\rho, x_{i}, y_{i}\right)$ pour $i \in[1, t], t$ étant un entier convenable et l'ordre croissant des $i$ correspondant à l'ordre croissant dans l'ordre fixé. On récrit:

$$
\pi_{0} \hookrightarrow \times_{(\tau, x, y) ; \tau \neq \rho} S D(\tau, x, y) \quad \times_{i \in[1, t]} S D\left(\rho, x_{i}, y_{i}\right) \times \pi_{\text {cusp }} .
$$

Fixons $i_{0} \in[1, t]$ et $k \in\left[x_{i_{0}}, y_{i_{0}}\right]$; on a:

$$
S D\left(\rho, x_{i_{0}}, y_{i_{0}}\right) \hookrightarrow S D\left(\rho, x_{i_{0}}, k+1\right) \times S D\left(\rho, k, y_{i_{0}}\right),
$$

où $S D\left(\rho, x_{i_{0}}, k+1\right)$ n'intervient pas si $k=x_{i_{0}}$. On glisse cette inclusion dans le terme de droite de (1') et on voit qu'il existe un sous-quotient $\pi_{i_{0}, k}$ de l'induite:

$$
S D\left(\rho, k, y_{i}\right) \times_{i>i_{0}} S D\left(\rho, x_{i}, y_{i}\right) \times \pi_{c u s p},
$$

tel que

$\pi_{0} \hookrightarrow \times_{(\tau, x, y) ; \tau \neq \rho} S D(\tau, x, y) \quad \times_{i \in\left[1, i_{0}[\right.} S D\left(\rho, x_{i}, y_{i}\right) \times S D\left(\rho, x_{i_{0}}, k+1\right) \times \pi_{i_{0}, k}$.

Comme $\pi_{0}$ est fortement positive, $\pi_{i_{0}, k}$ est nécessairement une série discrète fortement positive.

Notons $a_{\rho}$ le plus grand entier tel que $\left(\rho, a_{\rho}\right) \in \operatorname{Jord}\left(\pi_{\text {cusp }}\right)$; on pose $a_{\rho}=0 \mathrm{si}$ cet ensemble est vide mais que $L(\rho, R, s)$ a un pôle en $s=0$ et on remarque tout de suite que dans le cas où l'ensemble est vide alors que $L(\rho, R, s)$ n'a pas de pôle en $s=0$ alors $\rho||^{y_{t}} \times \pi_{\text {cusp }}$ est irréductible isomorphe à $\left.\rho\right|^{-y_{t}} \times \pi_{c u s p}$ ce qui est impossible. Donc sous ces hypothèses sur $\rho$ et $\pi_{c u s p}$, on est sûr qu'il n'intervient pas de triplet $(\rho, x, y)$ dans (1).

On va démontrer par récurrence décroissante sur $i_{0}$ et croissante sur $k$ les propriétés suivantes:

$$
\begin{gathered}
y_{i}=\left(a_{\rho}-2\left(t-i_{0}+1\right)+1\right) / 2, \\
x_{i}<x_{i^{\prime}},
\end{gathered}
$$

pour $\left.\left.i, i^{\prime} \in\right] i_{0}, t\right]$ et $i<i^{\prime}$ ainsi que $k<x_{i_{0}+1}$ et

$$
\pi_{i_{0}, k} \hookrightarrow S D\left(\rho, k, y_{i_{0}}\right) \times_{i>i_{0}} S D\left(\rho, x_{i}, y_{i}\right) \times \pi_{\text {cusp }} \quad \quad\left(*_{i_{0}, k}\right)
$$

Les blocs de Jordan de $\pi_{i_{0}, k}$ sont ceux de $\pi_{\text {cusp }}$ sauf les $\left(\rho, a_{\rho}-2(t-i)\right)$ pour $i \in\left[i_{0}, t\right]$ qui deviennent $\left(\rho, 2 x_{i}+1\right)$ si $i>i_{0}$ et $(\rho, 2 k+1)$ si $i=i_{0}$. Quand on aura cela pour $i_{0}=1$ et $k=x_{1}$ (et pour tout $\rho$ ), on aura démontré la proposition. Remarquons d'ailleurs que les premières propriétés entraînent que l'induite $\left(*_{i_{0}, k}\right)$ 
a un unique sous-module. On va admettre ces propriétés pour $\pi_{i_{0}, k-1}$, en posant $\pi_{t, y_{t}-1}=\pi_{\text {cusp }}$ et $\pi_{i_{0}, y_{i_{0}}-1}=\pi_{i_{0}+1, x_{i_{0}+1}}$ si $i_{0}<t$. Et on va les démontrer pour $\pi_{i_{0}, k}$.

Par construction, $\pi_{i_{0}, k}$ est un sous-quotient de

$$
\rho||^{k} \times \pi_{i_{0}, k-1} .
$$

Et pour $(\rho, a)$ comme d'habitude, on calcule $d\left(\rho, a, \pi_{i_{0}, k}, s\right)$ à l'aide de l'induite qui vient d'être écrite; on trouve que $d\left(\rho, a, \pi_{i_{0}, k}, s\right)$ est à une fonction holomorphe inversible près:

$$
\begin{gathered}
d\left(\rho, a, \pi_{i_{0}, k-1}, s\right) \times \\
L(S D(\rho, a) \times \rho, s-k) L(S D(\rho, a) \times \rho, s-k+1)^{-1} \times \\
L(S D(\rho, a) \times \rho, k-s) L(S D(\rho, a) \times \rho, k-s+1)^{-1} \times \\
L(S D(\rho, a) \times \rho,-s-k) L(S D(\rho, a) \times \rho,-s-k+1)^{-1} \times \\
L(S D(\rho, a) \times \rho, k+s) L(S D(\rho, a) \times \rho, k+s)^{-1} .
\end{gathered}
$$

On obtient:

$$
1 / 2 \operatorname{ordre}_{s=0} d\left(\rho, a, \pi_{i_{0}, k}\right)=1 / 2 \text { ordre }_{s=0} d\left(\rho, a, \pi_{i_{0}, k-1}\right)+\lambda,
$$

où $\lambda=1$ si $k=(a+1) / 2, \lambda=-1$ si $k=(a-1) / 2$ et $\lambda=0$ sinon. Or

$$
1 / 2 \operatorname{ordre}_{s=0} d\left(\rho, a, \pi_{i_{0}, k-1}\right)=\eta_{\rho, a}+\left|\left\{\left(\rho, a^{\prime}\right) \in \operatorname{Jord}\left(\pi_{i_{0}, k-1}\right) ; a^{\prime}=a\right\}\right|
$$

où $\eta_{\rho, a}=0$ sauf si $a$ est paire (resp. impaire) alors que $L(\rho, R, s)$ n'a pas de (resp. a un) pôle en $s=0$, où il vaut -1 . Or on sait que le nombre cherché vaut 0 ou -1 , d'après ce que l'on a rappelé. En faisant $a=2 x_{i}+1$ pour $\left.\left.i \in\right] i_{0}, t\right]$ (ce qui correspond à l'un des blocs de Jordan de $\pi_{i_{0}, k-1}$ ), on voit que l'on a certainement $k \neq x_{i}$ pour $\left.\left.i \in\right] i_{0}, t\right]$. De même, en faisant $a=a_{\rho}-2(t-i)$ pour $i<i_{0}$, on voit que $k \neq\left(a_{\rho}-2(t-i)-1\right) / 2$ pour ces valeurs de $i$. Comme on a déjà vu que $k-a_{\rho} \in \mathbf{Z}$, il ne reste en fait plus qu'à démontrer que si $k=y_{i_{0}}$ alors $k=y_{i_{0}}=\left(a_{\rho}-2\left(t-i_{0}\right)+1\right) / 2$. On prend ici $a$ tel que $(a+1) / 2=y_{i_{0}}$. Comme l'odre en $s=0 \operatorname{de} d\left(\rho, a, \pi_{i_{0}, y_{i_{0}}}\right)$ n'est pas strictement positif, on doit avoir:

$$
\left(\rho, 2 y_{i_{0}}-1\right) \in \operatorname{Jord}\left(\pi_{i_{0}+1, x_{i_{0}+1}}\right) .
$$

Ce bloc de Jordan n'est certainement pas de la forme $\left(\rho, a_{\rho}-2(t-i)\right)$ pour $i<i_{0}$ d'après ce que l'on a déjà vu. C'est donc soit $\left(\rho, a_{\rho}-2\left(t-i_{0}\right)\right)$, ce que l'on cherche, soit $\left(\rho, 2 x_{i}+1\right)$ pour $i>i_{0}$. Ce dernier cas est exclu car on a:

$$
2 y_{i_{0}}-1 \leq 2 y_{i}-1<2 x_{i}+1 \text {. }
$$

Cela termine la récurrence et la preuve. 
4.2 Blocs de Jordan pour une série discrète générale

Soit $\pi_{0}$ une série discrète; on a vu qu'il existe une série discrète fortement positive $\pi_{+}$et un ensemble de triplets $(\tau, x, y)$, tels que:

$$
\pi_{0} \hookrightarrow \times_{(\tau, x, y)} S D(\tau, x,-y) \times \pi_{+} .
$$

Il n’y a absolument pas unicité de telles données.

Proposition: $\operatorname{Jord}\left(\pi_{0}\right)$ est la réunion de $\operatorname{Jord}\left(\pi_{+}\right)$et de l'ensemble $\{(\tau, 2 x+1)$; $(\tau, 2 y+1)\}$ pour $(\tau, x, y)$ parcourant l'ensemble des triplets intervenant dans $(4)$; ces deux ensembles sont disjoints.

On montre comme dans la proposition précédente que les $x$ et $y$ intervenant sont nécessairement des demi-entiers et on peut même préciser qu'ils sont précisément entiers si $L(\rho, R, s)$ n'a pas de pôle en $s=0$. Pour $(\rho, a)$ comme d'habitude, on calcule $d\left(\rho, a, \pi_{0}, s\right)$ en utilisant (4). A une fonction holomorphe inversible près, cela vaut:

$$
\begin{gathered}
L(S D(\rho, a) \times \tau, s-x) L(S D(\rho, a) \times \tau, s+y+1)^{-1} \times \\
L(S D(\rho, a) \times \tau,-y+s) L(S D(\rho, a) \times \tau, x+s+1)^{-1} \times \\
L(S D(\rho, a) \times \tau,-s-x) L(S D(\rho, a) \times \tau,-s+y+1)^{-1} \times \\
L(S D(\rho, a) \times \tau,-y-s) L(S D(\rho, a) \times \tau, x-s+1)^{-1} \times \\
d\left(\rho, a, \pi_{+}, s\right) .
\end{gathered}
$$

D’où, avec $\eta_{\rho, a}$ comme dans la preuve précédente:

$$
\begin{gathered}
1 / 2 \operatorname{ordre}_{s=0} d\left(\rho, a, \pi_{0}, s\right)=\eta_{\rho, a} \\
+|\{(\tau, x, y) ; \tau \simeq \rho ; x=(a-1) / 2\}|+|\{(\tau, x, y) ; \tau \simeq \rho ; y=(a-1) / 2\}| \\
+\left|\left\{\left(\rho^{\prime}, a^{\prime}\right) \in \operatorname{Jord}\left(\pi_{+}\right) ;\left(\rho^{\prime}, a^{\prime}\right)=(\rho, a)\right\}\right| .
\end{gathered}
$$

Cela donne le résultat et en particulier l'absence de multiplicité puisque l'ordre est 0 ou -2 .

\section{Le caractère associé aux séries discrètes}

5.1 Soit $\pi_{0}$ une série discrète; on définit ici partiellement la fonction

$$
\epsilon_{\pi_{0}}: \operatorname{Jord}\left(\pi_{0}\right) \rightarrow\{ \pm 1\} .
$$

Pour $a, a^{\prime} \in \mathbf{N}$ tels que $(\rho, a)$ et $\left(\rho, a^{\prime}\right)$ sont dans $\operatorname{Jord}\left(\pi_{0}\right)$, on définit la "différence" $\epsilon_{\pi_{0}}(\rho, a) \epsilon_{\pi_{0}}\left(\rho, a^{\prime}\right)^{-1}$. Pour cela il suffit de le faire pour les couples $\left(a, a^{\prime}\right)$ tels que $a>a^{\prime}$ et pour tout $\left.b \in\right] a, a^{\prime}\left[,(\rho, b) \notin \operatorname{Jord}\left(\pi_{0}\right)\right.$; dans cette situation on note $a^{\prime}$ par $a_{-}$. On pose:

$$
\epsilon_{\pi_{0}}(\rho, a)=\epsilon_{\pi_{0}}\left(\rho, a_{-}\right) \Leftrightarrow
$$

il existe une représentation irréductible $\pi^{\prime}$ de $G\left(n-d_{\rho}\left(a-a_{-}\right) / 2, F\right)$ et une inclusion:

$$
\pi_{0} \hookrightarrow S D\left(\rho,(a-1) / 2,\left(a_{-}+1\right) / 2\right) \times \pi^{\prime} .
$$


Dans le cas où $L(\rho, R, s)$ a un pôle en $s=0$, on peut compléter cette définition en notant $a_{\text {min }}$ le plus petit entier tel que $\left(\rho, a_{\text {min }}\right) \in \operatorname{Jord}\left(\pi_{0}\right)$; c'est un nombre pair. Et on pose:

$$
\epsilon_{\pi_{0}}\left(\rho, a_{\min }\right)=1 \Leftrightarrow
$$

il existe une représentation irréductible de $G\left(n-d_{\rho} a_{\min } / 2, F\right)$ et une inclusion:

$$
\pi_{0} \hookrightarrow S D(\rho,(a-1) / 2,1 / 2) \times \pi^{\prime} .
$$

On complètera un peu la définition de $\epsilon_{\pi_{0}}$ plus loin.

Lemme: Dans la situation de (1) en supposant que $\epsilon_{\pi_{0}}(\rho, a)=\epsilon_{\pi_{0}}\left(\rho, a_{-}\right)$, $\pi^{\prime}$ est nécessairement un sous-module d'une induite du type:

$$
S D\left(\rho, a_{-}\right) \times \pi_{0}^{\prime},
$$

où $\pi_{0}^{\prime}$ est une série discrète dont l'ensemble des blocs de Jordan est exactement celui de $\pi_{0}$ dont on a enlevé $(\rho, a)$ et $\left(\rho, a_{-}\right)$. Ainsi:

$$
\pi_{0} \hookrightarrow S D\left(\rho,(a-1) / 2,-\left(a_{-}-1\right) / 2\right) \times \pi_{0}^{\prime} .
$$

On montre d'abord que $\pi^{\prime}$ est nécessairement tempérée; s'il n'en est pas ainsi, on a montré dans la preuve de 3.1 (cela résulte aussi de la classification de Langlands), qu'il existe $\tau, x, y$ (ce sont les notations usuelles) tels que:

$$
\pi^{\prime} \hookrightarrow S D(\tau, x, y) \times \pi^{\prime \prime},
$$

où $\pi^{\prime \prime}$ est une représentation convenable et où:

$$
\sum_{k \in[x, y]} k<0 .
$$

En reportant dans (1), on obtient:

$$
\pi_{0} \hookrightarrow S D\left(\rho,(a-1) / 2,\left(a_{-}+1\right) / 2\right) \times S D(\tau, x, y) \times \pi^{\prime \prime} .
$$

On ne peut pas remplacer $S D\left(\rho,(a-1) / 2,\left(a_{-}+1\right) / 2\right) \times S D(\tau, x, y)$ par $S D(\tau, x, y)$ $\times S D\left(\rho,(a-1) / 2,\left(a_{-}+1\right) / 2\right)$ que si $\tau \simeq \rho$ et $\left.\left.x \in\right](a-1) / 2,\left(a_{-}-1\right) / 2\right]$ (on utilise ici le fait que $y \leq 0)$; si on peut faire cette échange, on aurait une contradiction avec le fait que $\pi_{0}$ est une série discrète. Plaçons nous dans le cas où on ne peut échanger. L'induite a un sous-module:

$$
S D(\rho,(a-1) / 2, y) \times S D\left(\rho, x,\left(a_{-}+1\right) / 2\right)
$$

et un quotient qui est sous-module de $S D(\tau, x, y) \times S D\left(\rho,(a-1) / 2,\left(a_{-}+1\right) / 2\right)$. D'après ce que l'on vient de voir, nécessairement:

$$
\pi_{0} \hookrightarrow S D(\rho,(a-1) / 2, y) \times S D\left(\rho, x,\left(a_{-}+1\right) / 2\right) \times \pi^{\prime \prime} .
$$

On récrit cela:

$$
\pi_{0} \hookrightarrow S D(\rho,(a-1) / 2, y) \times \pi^{\prime \prime \prime}
$$


pour $\pi^{\prime \prime \prime}$ convenable. En outre on sait que $y<-\left(a_{-}-1\right) / 2$; c'est l'hypothèse de négativité couplée avec le fait que $x \geq\left(a_{-}-1\right) / 2$. On a montré en 3.2, qu'il existe donc $y^{\prime} \leq y$ et $\pi_{0}^{\prime}$ une série discrète telle que:

$$
\pi_{0} \hookrightarrow S D\left(\rho,(a-1) / 2, y^{\prime}\right) \times \pi_{0}^{\prime} .
$$

Nécessairement $-2 y^{\prime}+1<a$. On peut calculer les blocs de Jordan, en utilisant cette inclusion (cf. la preuve de 4.2):

$$
\operatorname{Jord}\left(\pi_{0}\right)=\left\{(\rho, a) ;\left(\rho,-2 y^{\prime}+1\right)\right\} \cup \operatorname{Jord}\left(\pi_{0}^{\prime}\right) .
$$

Comme $-2 y^{\prime}+1>a_{-}$cela contredit la définition de $a_{-}$.

Puisque l'on a démontré que $\pi^{\prime}$ est une représentation tempérée, on écrit $\pi^{\prime}$ sous la forme d'un sous-module d'une induite du type:

$$
\times{ }_{\left(\rho^{\prime}, a^{\prime}\right)} S D\left(\rho^{\prime}, a^{\prime}\right) \times \pi_{0}^{\prime \prime},
$$

où $\left(\rho^{\prime}, a^{\prime}\right)$ parcourt un ensemble convenable et où $\pi_{0}^{\prime \prime}$ est une série discrète. D'où:

$$
\pi_{0} \hookrightarrow S D\left(\rho,(a-1) / 2,\left(a_{-}+1\right) / 2\right) \times{ }_{\left(\rho^{\prime}, a^{\prime}\right)} S D\left(\rho^{\prime}, a^{\prime}\right) \times \pi_{0}^{\prime \prime} .
$$

On calcule les blocs de Jordan de $\pi_{0}$ en utilisant cette inclusion (ce que l'on ne refait pas) et on en déduit le lemme sauf la fin qui est à peu près évidente. On préfère démontrer les 2 remarques très utiles:

5.1.1 Remarque: Dans la situation de 5.1, la représentation:

$$
S D\left(\rho,(a-1) / 2,-\left(a_{-}-1\right) / 2\right) \times \pi_{0}^{\prime}
$$

a exactement 2 sous-modules irréductibles.

Pour démontrer cette remaque, on va utiliser, ainsi qu'à de nombreux autres endroits cette autre remarque:

5.1.2 Remarque: Soit $\pi_{0}$ une série discrète de $G(n, F)$ et soient $\rho$ une représentation cuspidale unitaire de $G L\left(d_{\rho}, F\right), x \in \mathbf{R}$ tels qu'il existe une représentation $\sigma$ de $G\left(n-d_{\rho}, F\right)$ et une inclusion:

$$
\pi_{0} \hookrightarrow \rho||^{x} \times \sigma .
$$

Alors il existe $a \in \mathbf{N}$ tel que $(\rho, a) \in \operatorname{Jord}\left(\pi_{0}\right)$ et $x=(a-1) / 2$.

On démontre d'abord 5.1.2. Soit $x$ et $\sigma$ comme dans l'énoncé; on sait a priori que $x>0$ car $\pi_{0}$ est une série discrète. Pour conclure, on utilise alors les formules de Bernstein-Zelevinski pour calculer les modules de Jacquet des induites que l'on applique à 4.2 et 4.1 .

On montre maintenant 5.1.1. On calcule l'occurence du terme:

$$
\otimes_{i \in\left[(a-1) / 2,-\left(a_{-}-1\right) / 2 \rho||^{i} \times \pi_{0}^{\prime}\right.}
$$


comme sous-quotient du module de Jacquet de l'induite écrite. Montrons qu'il intervient exactement avec multiplicité 2; c'est là que l'on utilise 5.1.2 et le fait que pour tout $b \in] a, a_{-}\left[,(\rho, b) \notin \operatorname{Jord}\left(\pi_{0}^{\prime}\right)\right.$. En effet, si son occurence est strictement supérieur à 2, les formules de Bernstein-Zelevinski assurent que soit il existe $j \in\left[(a-1) / 2,\left(a_{-}+1\right) / 2\right]$ et une inclusion

$$
\pi_{0}^{\prime} \hookrightarrow \rho||^{j} \times \sigma^{\prime}
$$

pour $\sigma^{\prime}$ convenable, soit il existe $j \in\left[(a-1) / 2,-\left(a_{-}-1\right) / 2\right.$ et une inclusion:

$$
\pi_{0}^{\prime} \hookrightarrow \times_{i \in\left[j,-\left(a_{-}-1\right) / 2\right]} \rho||^{i} \times \sigma^{\prime \prime},
$$

pour $\sigma^{\prime \prime}$ convenable. Le fait que $\pi_{0}^{\prime}$ est une série discrète force $j>\left(a_{-}-1\right) / 2$ dans le deuxième cas qui est alors une condition encore plus forte que le premier cas. Ils contredisent 5.1.1. Il reste encore à vérifier que l'induite a exactement 2 sous-modules irréductibles. Mais le fait que $\left(\rho, a_{-}\right) \notin \operatorname{Jord}\left(\pi_{0}^{\prime}\right)$ assure que $S D\left(\rho, a_{-}\right) \times \pi_{0}^{\prime}$ est réductible donc de longueur 2 . Soit $\tau_{\epsilon}$ les 2 sous-modules de cet induite. Chaque induite:

$$
S D\left(\rho,(a-1) / 2,\left(a_{-}+1\right) / 2\right) \times \tau_{\epsilon}
$$

a (exactement) un sous-module irréductible. Le module de Jacquet de ce sousmodule a comme facteur direct $S D\left(\rho,(a-1) / 2,-\left(a_{-}-1\right) / 2\right) \times \pi_{0}^{\prime}$ (cela se voit en regardant le support cuspidal; c'est le seul terme ayant ce support cuspidal). Le sous-module considéré est donc aussi sous-module de l'induite:

$$
S D\left(\rho,(a-1) / 2,-\left(a_{-}-1\right) / 2\right) \times \pi_{0}^{\prime} .
$$

D’où la remarque.

5.1.3 Remarque: Soit $\pi_{0}$ une série discrète et $(\rho, a) \in \operatorname{Jord}\left(\pi_{0}\right)$. On suppose que $a_{-}$est défini comme en 5.1. Les propriétés suivantes sont équivalentes:

1-il existe une représentation $\sigma$ de $G\left(n-\left(a-a_{-}\right) / 2, F\right)$ et une inclusion:

$$
\pi_{0} \hookrightarrow \times_{i \in\left[(a-1) / 2,\left(a_{-}+1\right) / 2\right]} \rho||^{i} \times \sigma
$$

2-il existe une représentation $\sigma^{\prime}$ de $G\left(n-\left(a-a_{-}\right) / 2, F\right)$ et une inclusion:

$$
\pi_{0} \hookrightarrow S D\left(\rho,(a-1) / 2,\left(a_{-}+1\right) / 2\right) \times \sigma^{\prime} .
$$

3-il existe une série discrète $\pi_{0}^{\prime}$ de $G\left(n-d_{\rho}\left(a+a_{-}\right) / 2, F\right)$ telle que $\operatorname{Jord}\left(\pi_{0}^{\prime}\right)=$ $\operatorname{Jord}\left(\pi_{0}\right)-\left\{(\rho, a),\left(\rho, a_{-}\right)\right\}$et une inclusion:

$$
\pi_{0} \hookrightarrow S D\left(\rho,(a-1) / 2,-\left(a_{-}-1\right) / 2\right) \times \pi_{0}^{\prime} .
$$


La deuxième propriété entraîne trivialement la première; mais la première entraîne la deuxième: il existe un sous-quotient $\tau$ de $\times_{i \in\left[(a-1) / 2,\left(a_{-}+1\right) / 2\right]} \rho^{i}$ vue comme représentation de $G L\left(d_{\rho}\left(a-a_{-}\right) / 2, F\right)$ avec une inclusion:

$$
\pi_{0} \hookrightarrow \tau \times \sigma .
$$

On connait parfaitement bien les modules de Jacquet de $\tau$ en tant que représentation d'un groupe linéaire. Si $\tau$ n'est pas la représentation $S D\left(\rho,(a-1) / 2,\left(a_{-}+1\right) / 2\right)$ alors il existe $\left.j \in](a-1) / 2,\left(a_{-}+1\right) / 2\right]$ et $j^{\prime}$ dans le même ensemble avec $j^{\prime} \leq j$ et une inclusion:

$$
\tau \hookrightarrow S D\left(\rho, j, j^{\prime}\right) \times \tau^{\prime},
$$

où $\tau^{\prime}$ est convenable. Il y aurait alors une inclusion:

$$
\pi_{0} \hookrightarrow \rho||^{j} \times \sigma^{\prime \prime},
$$

avec $\sigma^{\prime \prime}$ convenable ce qui contredit 5.1.2.

La troisième propriété entraîne trivialement la $2 \mathrm{e}$. Il résulte de 5.1 que la $2 \mathrm{e}$ propriété entraîne une inclusion:

$$
\pi_{0} \hookrightarrow S D\left(\rho,(a-1) / 2,\left(a_{-}+1\right) / 2\right) \times S D\left(\rho, a_{-}\right) \times \pi_{0}^{\prime} .
$$

La représentation $S D\left(\rho,(a-1) / 2,\left(a_{-}+1\right) / 2\right) \times S D\left(\rho, a_{-}\right)$de $G L\left(d_{\rho}(a+\right.$ $\left.a_{-}\right) / 2, F$ ) est de longueur 2 . Si on ne peut la remplacer par son unique sousmodule irréductible, on vérifie par les formules de Bernstein-Zelevinski (avec la remarque 5.1.2 appliquée à $\pi_{0}^{\prime}$ ) que l'on ne trouverait pas dans le module de Jacquet de $\pi_{0}^{\prime}$ de terme:

$$
\otimes_{i \in\left[(a-1) / 2,\left(a_{-}+1\right) / 2\right]} \rho||^{i} \otimes \cdots .
$$

Ce qui est bien sûr absurde.

5.2 Lemme: Soit $\pi_{0}$ une série discrète; on suppose qu'il existe $(\rho, x, y)$ un triplet comme en 4.2 et une série discrète $\pi_{0}^{\prime}$ de $G\left(n-d_{\rho}(x+y+1), F\right)$ tel que:

$$
\pi_{0} \hookrightarrow S D(\rho, x,-y) \times \pi_{0}^{\prime} .
$$

Alors $\operatorname{Jord}\left(\pi_{0}\right)=\operatorname{Jord}\left(\pi_{0}^{\prime}\right) \cup\{(\rho, 2 x+1),(\rho, 2 y+1)\}$; en particulier, en définissant $(2 x+1)_{-}$comme le plus grand élément vérifiant $\left.\left.(2 x+1)_{-} \in\right] 2 x+1,2 y+1\right]$ et $(\rho, 2 x+1) \in \operatorname{Jord}\left(\pi_{0}\right)$, on a:

$$
\epsilon_{\pi_{0}}(\rho, 2 x+1)=\epsilon_{\pi_{0}}\left(\rho,(2 x+1)_{-}\right) .
$$

On peut en fait démontrer plus: pour tout $b \in[2 y+1,2 x+1]$ tel que $(\rho, b) \in$ $\operatorname{Jord}\left(\pi_{0}\right)$ :

$$
\epsilon_{\pi_{0}}(\rho, 2 x+1)=\epsilon_{\pi_{0}}(\rho, b) .
$$

Mais on ne l'utilisera pas et on ne le démontre donc pas.

Le lemme est facile; le calcul de $\operatorname{Jord}\left(\pi_{0}\right)$ a déjà été fait en 4.2. Et pour avoir la deuxième assertion, il suffit de remarquer l'inclusion:

$$
S D(\rho, x,-y) \hookrightarrow S D\left(\rho, x,\left(a_{-}+1\right) / 2\right) \times S D\left(\rho,\left(a_{-}+1\right),-y\right) .
$$

La variante plus complète est elle beaucoup plus longue à démontrer.

On a maintenant la caractérisation des séries discrètes fortement positives: 
5.3 Proposition: Soit $\pi_{0}$ une série discrète; alors $\pi_{0}$ est fortement positive si et seulement si pour tout $(\rho, a) \in \operatorname{Jord}\left(\pi_{0}\right)$ tel que $a_{-}$soit défini:

$$
\epsilon_{\pi_{0}}(\rho, a) \neq \epsilon_{\pi_{0}}\left(\rho, a_{-}\right) .
$$

Montrons que la condition est suffisante. Soit $\pi_{0}$ une série discrète et supposons qu'elle ne soit pas fortement positive, on va montrer qu'il existe $\rho, a, a_{-}$tel que

$$
\epsilon_{\pi_{0}}(\rho, a)=\epsilon_{\pi_{0}}\left(\rho, a_{-}\right) .
$$

En effet, on a montré qu'il existe un triplet $\tau, x, y$ et une série discrète $\pi_{0}^{\prime}$ tel que:

$$
\pi_{0} \hookrightarrow S D(\tau, x,-y) \times \pi_{0}^{\prime} .
$$

On pose $a=2 x+1$, alors $a_{-}$est défini et on a montré ci-dessus que cela entraîne que:

$$
\epsilon_{\pi_{0}}(\rho, a)=\epsilon_{\pi_{0}}\left(\rho, a_{-}\right) .
$$

Montrons maintenant que la condition est nécessaire; supposons qu'elle ne soit pas réalisée. On fixe alors $(\rho, a) \in \operatorname{Jord}\left(\pi_{0}\right)$ tel que $a_{-}$soit défini et

$$
\epsilon_{\pi_{0}}(\rho, a)=\epsilon_{\pi_{0}}\left(\rho, a_{-}\right) .
$$

Du lemme 5.1, il résulte qu'il existe une série discrète $\pi_{0}^{\prime}$ et une inclusion:

$$
\pi_{0} \hookrightarrow S D\left(\rho,(a-1) / 2,\left(a_{-}+1\right) / 2\right) \times S D\left(\rho, a_{-}\right) \times \pi_{0}^{\prime} .
$$

Cela contredit la positivité forte.

5.4 Lemme: Soit $\pi_{0}$ une série discrète et soit $(\rho, a) \in \operatorname{Jord}\left(\pi_{0}\right)$; on suppose que $a_{-}$est défini et on note $\pi_{0}^{\prime}$ une série discrète telle que:

$$
\pi_{0} \hookrightarrow S D\left(\rho,(a-1) / 2,-\left(a_{-}-1\right) / 2\right) \times \pi_{0}^{\prime},
$$

Soient $(\rho, b)$ et $\left(\rho, b^{\prime}\right)$ des éléments de $\operatorname{Jord}\left(\pi_{0}^{\prime}\right)$; alors:

$$
\epsilon_{\pi_{0}^{\prime}}(\rho, b)=\epsilon_{\pi_{0}^{\prime}}\left(\rho, b^{\prime}\right) \Leftrightarrow \epsilon_{\pi_{0}}(\rho, b)=\epsilon_{\pi_{0}}\left(\rho, b^{\prime}\right) .
$$

C'est le lemme clé pour toutes les récurrences et il est long à démontrer. Sa démonstration est complètement élémentaire, c'est-à-dire n'utilise que des calculs de modules de Jacquet; c'est sans doute pour cela qu'il est si pénible à démontrer.

Il suffit de le démontrer quand $b^{\prime}=b_{-}$mais cette notion étant relative à $\pi_{0}^{\prime}$; on suppose qu'il en est ainsi mais on garde la notation $b^{\prime}$ car $(\rho, b)$ ne sont pas nécessairement adjacents dans $\operatorname{Jord}\left(\pi_{0}\right)$. Le lemme est vraiment facile si $b^{\prime}>a$ ou si $b<a$, qui sont exactement les cas où $(\rho, b)$ et $\left(\rho, b^{\prime}\right)$ sont des blocs de Jordan adjacents dans $\operatorname{Jord}\left(\pi_{0}\right)$. Avec les propriétés d'adjacence, il reste à voir le cas:

$$
b>a>a_{-}>b^{\prime} .
$$

Dans cette situation et pour $\pi_{0}$, on a:

$$
a=b_{-}, b^{\prime}=\left(a_{-}\right)_{-} .
$$


On va d'abord démontrer le lemme en supposant que

$$
\epsilon_{\pi_{0}}\left(\rho, a_{-}\right)=\epsilon_{\pi_{0}}\left(\rho, b^{\prime}\right) .
$$

Sous cette hypothèse, il existe une série discrète $\tau_{0}$ et une inclusion (cf le lemme 5.1):

$$
\pi_{0} \hookrightarrow S D\left(\rho,\left(a_{-}-1\right) / 2,-\left(b^{\prime}-1\right) / 2\right) \times \tau_{0} .
$$

On a par hypothèse:

$$
\pi_{0} \hookrightarrow S D\left(\rho,(a-1) / 2,-\left(a_{-}-1\right) / 2\right) \times \pi_{0}^{\prime} .
$$

Un calcul de module de Jacquet montre que:

$$
\tau_{0} \hookrightarrow S D\left(\rho,(a-1) / 2,\left(b^{\prime}+1\right) / 2\right) \times \pi_{0}^{\prime} .
$$

Montrons que cette inclusion donne l'équivalence:

$$
\epsilon_{\tau_{0}}(\rho, a)=\epsilon_{\tau_{0}}(\rho, b) \Leftrightarrow \epsilon_{\pi_{0}^{\prime}}\left(\rho, b^{\prime}\right)=\epsilon_{\pi_{0}^{\prime}}(\rho, b) .
$$

L'implication $\Leftarrow$ résulte de l'irréductibilité de l'induite:

$$
S D\left(\rho,(a-1) / 2,\left(b^{\prime}+1\right) / 2\right) \times S D\left(\rho,(b-1) / 2,\left(b^{\prime}+1\right) / 2\right) .
$$

Supposons donc que $\epsilon_{\tau_{0}}(\rho, a)=\epsilon_{\tau_{0}}(\rho, b)$. Cela entraîne qu'il existe une série discrète $\pi_{0}^{\prime \prime}$ et une inclusion:

$$
\tau_{0} \hookrightarrow S D(\rho,(b-1) / 2,-(a-1) / 2) \times \pi_{0}^{\prime \prime} .
$$

On revient à (1) qui donne un terme très particulier du module de Jacquet de $\tau_{0}$ qui doit apparaître dans le module de Jacquet de (3). Cela donne:

$$
\pi_{0}^{\prime} \hookrightarrow S D\left(\rho,(b-1) / 2,-\left(b^{\prime}-1\right) / 2\right) \times \pi_{0}^{\prime \prime} .
$$

Ce qui est bien l'implication de (2) cherchée.

Comme le segment (à la Zelevinski) $\left[\left(a_{-}-1\right) / 2,-\left(b^{\prime}-1\right) / 2\right]$ est inclus dans le segment $[(b-1) / 2,-(a-1) / 2]$, on a aussi l'implication:

$$
\epsilon_{\tau_{0}}(\rho, b)=\epsilon_{\tau_{0}}(\rho, a) \Rightarrow \epsilon_{\pi_{0}}(\rho, b)=\epsilon_{\pi_{0}}(\rho, a) .
$$

L'implication opposée se démontre en utilisant le fait que

$$
\pi_{0} \hookrightarrow S D(\rho,(b-1) / 2,(a+1) / 2) \times \pi^{\prime}
$$

seulement si une telle relation est vraie pour $\tau_{0}$. On a donc démontré:

$$
\epsilon_{\pi_{0}^{\prime}}\left(\rho, b^{\prime}\right)=\epsilon_{\pi_{0}^{\prime}}(\rho, b) \Leftrightarrow \epsilon_{\pi_{0}}(\rho, b)=\epsilon_{\pi_{0}}(\rho, a) .
$$

Mais le lemme 5.2 montre que l'on a:

$$
\epsilon_{\pi_{0}}(\rho, a)=\epsilon_{\pi_{0}}\left(\rho, a_{-}\right)
$$


et comme par hypothèse

$$
\epsilon_{\pi_{0}}\left(\rho, a_{-}\right)=\epsilon_{\pi_{0}}\left(\rho, b^{\prime}\right),
$$

on a bien l'énoncé du lemme dans ce cas.

L'hypothèse $\epsilon_{\pi_{0}}(\rho, a)=\epsilon_{\pi_{0}}(\rho, b)$ donne un raisonnement analogue. On suppose donc que

$$
\epsilon_{\pi_{0}}(\rho, a) \neq \epsilon_{\pi_{0}}(\rho, b)
$$

et que

$$
\epsilon_{\pi_{0}}\left(\rho, a_{-}\right) \neq \epsilon_{\pi_{0}}\left(\rho, b^{\prime}\right) .
$$

Comme $\epsilon_{\pi_{0}}(\rho, a)=\epsilon_{\pi_{0}}\left(\rho, a_{-}\right)$, on a, sous nos hypothèses:

$$
\epsilon_{\pi_{0}}(\rho, b)=\epsilon_{\pi_{0}}\left(\rho, b^{\prime}\right)
$$

Il faut donc démontrer que nos hypothèses entraînent aussi:

$$
\epsilon_{\pi_{0}^{\prime}}(\rho, b)=\epsilon_{\pi_{0}^{\prime}}\left(\rho, b^{\prime}\right)
$$

On raisonne donc de la façon suivante: on suppose que

$$
\epsilon_{\pi_{0}^{\prime}}(\rho, b) \neq \epsilon_{\pi_{0}^{\prime}}\left(\rho, b^{\prime}\right)
$$

et on démontre que soit

$$
\epsilon_{\pi_{0}}(\rho, a)=\epsilon_{\pi_{0}}(\rho, b)
$$

soit

$$
\epsilon_{\pi_{0}}\left(\rho, a_{-}\right)=\epsilon_{\pi_{0}}\left(\rho, b^{\prime}\right) .
$$

On démontre d'abord les 2 lemmes techniques suivants:

5.4.1 Lemme: Soit $\pi_{0}$ une série discrète et soit $\rho$ une représentation cuspidale unitaire, autoduale, d'un groupe linéaire $G L\left(d_{\rho}, F\right)$. Soit x un demi-entier supérieur ou égal à 1 . On suppose que pour tout $a \in \mathbf{N}$ tel que $(\rho, a) \in \operatorname{Jord}\left(\pi_{0}\right)$ on ait $x \neq(a+1) / 2$. Alors l'induite

$$
\rho||^{x} \times \pi_{0}
$$

est irréductible.

L'irréductibilité est aussi vrai si $x \in \mathbf{R}-1 / 2 \mathbf{Z}$ mais on n'en a pas besoin.

Ce lemme est certainement aussi démontré par Tadić mais avec un langage différent.

On sait que l'induite écrite a un unique quotient irréductible (le quotient de Langlands) et il suffit de démontrer que ce quotient est aussi un sous-module. Pour cela, on montre que l'opérateur d'entrelacement normalisé (comme expliqué en $\left.\left[\mathrm{M}_{1}\right]\right)$ :

$$
\rho||^{x} \times \pi_{0} \rightarrow \rho||^{-x} \times \pi_{0}
$$

est une bijection. Pour montrer cela, on le fait par récurrence: supposons que $\pi_{0}$ ne soit pas fortement positive. Alors, on a une inclusion, avec des choix convenables:

$$
\pi_{0} \hookrightarrow S D\left(\rho^{\prime},(a-1) / 2,-\left(a_{-}-1\right) / 2\right) \times \pi_{0}^{\prime} .
$$


Avec l'hypothèse faite les induites:

$$
S D\left(\rho^{\prime},(a-1) / 2,-\left(a_{-}-1\right) / 2\right) \times \rho||^{ \pm x},
$$

sont irréductibles. L'opérateur d'entrelacement normalisé qui leur est associé est alors une bijection $[\mathrm{M}-\mathrm{W}]$. En appliquant l'assertion à $\pi_{0}^{\prime}$, on obtient la bijectivité pour $\pi_{0}$.

Supposons que $\pi_{0}$ soit fortement positive. Si $\pi_{0}$ est cuspidale, c'est réglé par l'hypothèse de base faite dans l'introduction. Supposons donc que $\pi_{0}$ ne soit pas cuspidale. On a décrit ces représentations en 4.1; on reprend la description de loc.cit de $\pi_{0}$ comme l'unique sous-module de l'induite écrite, on change un peu les notations pour faire apparaître $\operatorname{Jord}\left(\pi_{0}\right)$ et non $\operatorname{Jord}\left(\pi_{\text {cusp }}\right)$ :

$$
\pi_{0} \hookrightarrow \times{ }_{\left(\rho^{\prime}, a^{\prime}\right) \in \operatorname{Jord}\left(\pi_{0}\right)} S D\left(\rho^{\prime},\left(a^{\prime}-1\right) / 2,\left(a^{\prime}-1\right) / 2-T_{\left(\rho^{\prime}, a^{\prime}\right)}\right) \times \pi_{c u s p},
$$

où la collection des $T_{\left(\rho^{\prime}, a^{\prime}\right)}$ a des propriétés très précises. On en déduit le fait suivant: soit $\left(\rho^{\prime}, a^{\prime}\right) \in \operatorname{Jord}\left(\pi_{0}\right)$ tel que soit $a_{-}^{\prime}$ soit défini et vérifie $a^{\prime}-a_{-}^{\prime}>2$. Alors il existe une série discrète $\pi_{0}^{\prime}$ telle que:

$$
\pi_{0} \hookrightarrow S D\left(\rho^{\prime},\left(a^{\prime}-1\right) / 2,\left(a_{-}^{\prime}+3\right) / 2\right) \times \pi_{0}^{\prime} .
$$

Si cela ne se produit pour aucun élément de $\operatorname{Jord}\left(\pi_{0}\right)$ soit $\pi_{0}$ est cuspidale soit il existe $\left(\rho^{\prime}, a^{\prime}\right)$ et un entier $x_{0}$ valant $1 / 2,1$ ou $3 / 2\left(x_{0}=1\right.$ si $a^{\prime}$ est impair, $1 / 2$ si $a^{\prime}$ est pair et $\epsilon_{\pi_{0}}\left(\rho^{\prime}, a^{\prime}\right)=1$ et $3 / 2$ dans le cas restant) tel que:

$$
\pi_{0} \hookrightarrow S D\left(\rho^{\prime},\left(a^{\prime}-1\right) / 2, x_{0}\right) \times \pi_{0}^{\prime} .
$$

Dans tous les cas, on a pris des hypothèses telles que les induites:

$$
S D\left(\rho^{\prime},\left(a^{\prime}-1\right) / 2,\left(a_{-}^{\prime}+3\right) / 2\right) \times \rho||^{ \pm x}
$$

et

$$
S D\left(\rho^{\prime},\left(a^{\prime}-1\right) / 2, x_{0}\right) \times \rho \|^{ \pm x}
$$

soient irréductibles. Pour conclure comme ci-dessus, il faut encore comprendre:

$$
\rho||^{x} \times \pi_{0}^{\prime}
$$

Le problème est que les blocs de Jordan de $\pi_{0}^{\prime}$ sont aussi des blocs de Jordan de $\pi_{0}$ sauf $\left(\rho^{\prime}, a_{-}^{\prime}+2\right)$ dans le cas (1) et dans le cas $(2)\left(\rho^{\prime}, \alpha\right)$ où $\alpha=1$ si $a^{\prime}$ est impair et 2 si $a^{\prime}$ est pair mais $\epsilon_{\pi_{0}}\left(\rho^{\prime}, a^{\prime}\right)=-1$. Le cas restant n'intervient pas ici. On a donc un problème si pour tout choix possible de $\left(\rho^{\prime}, a^{\prime}\right)$ comme ci-dessus:

$$
\rho^{\prime} \simeq \rho ; x=\left(a_{-}^{\prime}+3\right) / 2 .
$$

Cela veut dire qu'il n'y a qu'un choix possible que l'on note $(\rho, a)$. On pose $b:=2 x-1$; ainsi $x=(b+1) / 2$.

Traitons d'abord le cas où $a=b+2$ et où $a$ est maximal avec la propriété que $(\rho, a) \in \operatorname{Jord}\left(\pi_{0}\right)$; ce cas est traité par Tadić par une méthode n'utilisant pas les 
opérateurs d'entrelacement. On montrera plus bas que l'hypothèse de récurrence ne laisse que ce cas. Sous ces hypothèses, $\pi_{0}$ est l'unique sous-module de l'induite:

$$
\left.\rho\right|^{(a-1) / 2} \times \pi_{\text {cusp }}
$$

et $x=(a-1) / 2$. On note M(s) l'opérateur d'entrelacement standard:

$$
\rho||^{s} \times \pi_{0} \rightarrow \rho||^{-s} \times \pi_{0} .
$$

On a calculé le composé $M(-s) \circ M(s)$ en [M1] (sous les hypothèses que l'on a faites au début) en terme simple de fonction $L$ liées aux blocs de Jordan. On vérifie que ce composé à un pôle d'ordre 1 exactement en $s=(a-1) / 2$. Or l'opérateur:

$$
\begin{gathered}
\rho||^{-s} \times \rho||^{(a-1) / 2} \times \pi_{\text {cusp }} \\
\downarrow \\
\rho||^{s} \times \rho||^{(a-1) / 2} \times \pi_{\text {cusp }}
\end{gathered}
$$

se décompose en produit d'opérateurs d'entrelacement standard. Ils sont tous holomorphes en $s=(a-1) / 2$ sauf l'opérateur:

$$
\rho||^{(a-1) / 2} \times \rho||^{s} \rightarrow \rho||^{s} \times \rho||^{(a-1) / 2}
$$

qui a un pôle d'ordre 1 en $s=(a-1) / 2$. On pose $N^{\prime}(s):=(s-(a-1) / 2) M(s)$ et le composé:

$$
N^{\prime}(-s) \circ M(s)
$$

est holomorphe non nul en $s=(a-1) / 2$; comme chacun des opérateurs est lui aussi holomorphe en $s=(a-1) / 2$, ils sont inverses l'un de l'autre et l'on a l'irréductibilité cherchée.

Il nous reste à montrer comment on se ramène à ce cas. Supposons d'abord que $a>b+2$. Alors, il existe une série discrète $\pi_{1}$ tel que:

$$
\pi_{0} \hookrightarrow S D(\rho,(a-1) / 2,(b+1) / 2) \times \pi_{1} ;
$$

et il existe aussi $\pi_{1}^{\prime}$ une série discrète incluse dans l'induite $\left.\rho\right|^{(b+1) / 2} \times \pi_{1}$ (elle en est même l'unique sous-module) tel que:

$$
\pi_{0} \hookrightarrow S D(\rho,(a-1) / 2,(b+3) / 2) \times \pi_{1}^{\prime} .
$$

Le sous-module de $\left.\rho\right|^{(b+1) / 2} \times \pi_{0}$ (qui nous intéresse) est aussi un sous-module de:

$$
S D(\rho,(a-1) / 2,(b+1) / 2) \times \rho||^{(b+1) / 2} \times \pi_{1} .
$$

L'induite juste écrite a un unique sous-module; on calcule l'occurence de la représentation

$$
S D(\rho,(a-1) / 2,(b+3) / 2) \otimes\left(\rho||^{(b+1) / 2} \times \rho||^{(b+1) / 2}\right) \otimes \pi_{1}
$$

dans le module de Jacquet relativement au parabolique évident et cette occurence est 1 . Ainsi le sous-module de $\sigma$ de $\left.\rho\right|^{(b+1) / 2} \times \pi_{0}$ est aussi un sous-module de:

$$
S D(\rho,(a-1) / 2,(b+1) / 2) \times \pi_{1}^{\prime} .
$$


Comme on a supposé que $(a-1) / 2>(b+1) / 2$ par récurrence on sait que:

$$
\rho||^{(b+1) / 2} \times \pi_{1}^{\prime}
$$

est irréductible (les blocs de Jordan de $\pi_{1}^{\prime}$ ont les bonnes propriétés). Cela prouve que:

$$
\sigma \hookrightarrow S D(\rho,(a-1) / 2,(b+3) / 2) \times\left.\rho\right|^{-(b+1) / 2} \times \pi_{1}^{\prime} .
$$

Comme l'induite $S D(\rho,(a-1) / 2,(b+3) / 2) \times \rho||^{-(b+1) / 2}$ est irréductible, cela prouve encore qu'il existe une représentation $\pi^{\prime}$ tel que:

$$
\left.\sigma \hookrightarrow \rho\right|^{-(b+1) / 2} \times \pi^{\prime} .
$$

Cela entraîne que $\pi^{\prime} \simeq \pi_{0}$ (calcul des modules de Jacquet de $\rho||^{(b+1) / 2} \times \pi_{0}$ ).

On s'est maintenant ramené au cas où il n'y a qu'un saut dans les blocs de Jordan de $\pi_{0}$ qui n'est pas 2 et ce saut est de 4 ; on note $(\rho, a)$ le bloc de Jordan où se produit ce saut et on a:

$$
x=(b+1) / 2=(a-1) / 2 .
$$

En reprenant (*), il existe $t \in \mathbf{N}$ tel que:

$$
\pi_{0} \hookrightarrow \times_{i \in[1, t]} \rho||^{(a-1) / 2+i-1} \times \pi_{c u s p} .
$$

On note $\operatorname{Sp}(\rho,(a-1) / 2,(a-1) / 2+t-1)$ l'unique sous-module de l'induite:

$$
\times_{i \in[1, t]} \rho||^{(a-1) / 2+i-1}
$$

qui vit dans un groupe linéaire convenable. Et clairement:

$$
\pi_{0} \hookrightarrow \operatorname{Sp}(\rho,(a-1) / 2,(a-1) / 2+t-1) \times \pi_{c u s p} .
$$

On veut se ramener au cas où $t=1$; s'il n'en est pas ainsi:

$$
\rho||^{(a-1) / 2} \times \pi_{c u s p}
$$

est irréductible. De même les induites pour les groupes linéaires convenables:

$$
S p(\rho,(a-1) / 2,(a-1) / 2+t-1) \times \rho||^{(a-1) / 2}
$$

sont irréductibles et on peut donc raisonner comme on l'a déjà fait. Cela termine la preuve.

5.4.2 Lemme: Soit $\pi_{0}$ une série discrète et soit $(\rho, a) \in \operatorname{Jord}\left(\pi_{0}\right)$.

(i) on suppose ici que $a_{-}$est défini et vérifie $a-a_{-}>2$. Alors il existe une série discrète $\pi_{1}$ de $G\left(n-d_{\rho}\left(\left(a-a_{-}\right) / 2-1\right), F\right)$ telle que:

$$
\pi_{0} \hookrightarrow S D\left(\rho,(a-1) / 2,\left(a_{-}+3\right) / 2\right) \times \pi_{1} .
$$

(ii) on suppose ici que $a_{-}$n'est pas défini (i.e. que a est minimal avec la propriété $(\rho, a) \in \operatorname{Jord}\left(\pi_{0}\right)$ mais que $a>2$. Alors il existe une série discrète $\pi_{1}$ de $G\left(n-d_{\rho}([a / 2]-1), F\right)$ telle que:

$$
\pi_{0} \hookrightarrow S D(\rho,(a-1) / 2,(a-2[(a+1) / 2]+3) / 2) \times \pi_{1} .
$$


Dans les 2 cas, Jord $\left(\pi_{1}\right)$ contient l'élément $\left(\rho, a_{-}+2\right)$ ou $(\rho, a-2[(a+1) / 2]+2)$ si $a_{-} n^{\prime}$ est pas défini, ainsi qu'exactement tous les $\left(\rho^{\prime}, a^{\prime}\right) \in \operatorname{Jord}\left(\pi_{0}\right)$ tel que $\left(\rho^{\prime}, a^{\prime}\right) \neq(\rho, a)$.

Dans cette démonstration, pour unifier les deux cas, on pose, dans le cas où $a_{-}$ n'est pas définie:

$$
a_{-}:=a-2[(a+1) / 2] .
$$

Ce nombre vaut 0 si $a$ est pair et -1 sinon. On vérifie que si $a_{-}$n'était pas défini, on a:

$$
(a-1) / 2-[(a+1) / 2]+2=\left(a_{-}+3\right) / 2 .
$$

Ce nombre vaut 1 si $a$ est impair et $3 / 2$ si $a$ est pair.

On démontre d'abord qu'il existe une représentation $\pi^{\prime}$ de $G\left(n-d_{\rho}((a-\right.$ $\left.\left.a_{-}\right) / 2-1\right), F$ ) telle que:

$$
\pi_{0} \hookrightarrow S D\left(\rho,(a-1) / 2,\left(a_{-}+3\right) / 2\right) \times \pi^{\prime} .
$$

Si $\pi_{0}$ est fortement positive, cela se fait directement en utilisant 4.1 et les résultats de Zelevinski pour faire commuter des induites de groupes linéaires.

Si $\pi_{0}$ n'est pas fortement positive, on le fait par récurrence en utilisant 5.1: il existe $\left(\rho^{\prime}, b^{\prime}\right) \in \operatorname{Jord}\left(\pi_{0}\right)$ et une série discrète convenable, $\pi_{0}^{\prime}$ telle que $b_{-}^{\prime}$ soit défini et:

$$
\pi_{0} \hookrightarrow S D\left(\rho^{\prime},\left(b^{\prime}-1\right) / 2,-\left(b_{-}^{\prime}-1\right) / 2\right) \times \pi_{0}^{\prime} .
$$

On a décrit les blocs de Jordan de $\pi_{0}$ en fonction de $\rho^{\prime}, b^{\prime}, b_{-}^{\prime}$ et de ceux de $\pi_{0}^{\prime}$. Plusieurs cas sont possibles; le premier est facile, $(\rho, a) \neq\left(\rho^{\prime}, b_{-}^{\prime}\right)$. Ce cas se traite soit directement si $(\rho, a)=\left(\rho^{\prime}, b^{\prime}\right)$ soit par récurrence si $(\rho, a) \in \operatorname{Jord}\left(\pi_{0}^{\prime}\right)$. On a exactement pris les hypothèses qui assurent que l'induite:

$$
S D\left(\rho^{\prime},\left(b^{\prime}-1\right) / 2,-\left(b_{-}^{\prime}-1\right) / 2\right) \times S D\left(\rho,(a-1) / 2,\left(a_{-}+3\right) / 2\right)
$$

est irréductible. L'autre cas est celui où $(\rho, a)=\left(\rho^{\prime}, b^{\prime}\right)$. On écrira donc $\rho^{\prime}=\rho$ et $b^{\prime}=a$.

On sait que (1) a exactement 2 sous-modules irréductibles (un calcul de module de Jacquet plus le fait que $S D\left(\rho^{\prime}, b_{-}^{\prime}\right) \times \pi_{0}^{\prime}$ est de longueur 2$)$. On considère l'induite:

$$
S D\left(\rho,(b-1) / 2,-\left(a_{-}+3\right) / 2\right) \times S D\left(\rho,(a-1) / 2,\left(a_{-}+3\right) / 2\right) \times \pi_{0}^{\prime} .
$$

On vérifie avec les mêmes arguments que cette induite a elle aussi exactement 2 sous-modules irréductibles que nous notons $\tau_{\epsilon}$ pour $\epsilon= \pm 1$. Avec le lemme précédent, on vérifie que:

$$
S D\left(\rho,(a-1) / 2,\left(a_{-}+3\right) / 2\right) \times \pi_{0}^{\prime}
$$

est irréductible et donc isomorphe à l'induite:

$$
S D\left(\rho,-\left(a_{-}+3\right) / 2,-(a-1) / 2\right) \times \pi_{0}^{\prime} .
$$


Ainsi:

$\tau_{1} \oplus \tau_{-1} \hookrightarrow S D\left(\rho,(b-1) / 2,-\left(a_{-}+3\right) / 2\right) \times S D\left(\rho,-\left(a_{-}+3\right) / 2,-(a-1) / 2\right) \times \pi_{0}^{\prime}$.

Mais l'induite de droite a au plus 2 sous-modules irréductibles qui sont donc $\tau_{\epsilon}$. Elle contient aussi les sous-modules irréducibles de l'induite

$$
S D(\rho,(b-1) / 2,-(a-1) / 2) \times \pi_{0}^{\prime},
$$

puisque:

$$
\begin{gathered}
S D(\rho,(b-1) / 2,-(a-1) / 2) \hookrightarrow S D\left(\rho,(b-1) / 2,-\left(a_{-}+3\right) / 2\right) \times \\
S D\left(\rho,-\left(a_{-}+3\right) / 2,-(a-1) / 2\right) .
\end{gathered}
$$

Ainsi:

$$
\begin{aligned}
& \pi_{0} \hookrightarrow S D\left(\rho,(b-1) / 2,-\left(a_{-}+3\right) / 2\right) \times S D\left(\rho,(a-1) / 2,\left(a_{-}+3\right) / 2\right) \times \pi_{0}^{\prime} \\
& \quad \simeq S D\left(\rho,(a-1) / 2,\left(a_{-}+3\right) / 2\right) \times S D\left(\rho,(b-1) / 2,-\left(a_{-}+3\right) / 2\right) \times \pi_{0}^{\prime} .
\end{aligned}
$$

D'où le résultat qui prouve l'existence de $\pi^{\prime}$. En utilisant les méthodes de 3.1, on montre que $\pi^{\prime}$ est nécessairement une série discrète.

5.4.3 Fin de la démonstration de 5.4. On reprend les notations de 5.4 et on utilise le lemme 5.4.2, après avoir vérifié que $\left(b^{\prime}+3\right) / 2 \leq\left(a_{-}-2+3\right) / 2=\left(a_{-}+1\right) / 2$. On sait donc qu'il existe une série discrète $\pi_{1}^{\prime}$, telle que:

$$
\pi_{0}^{\prime} \hookrightarrow S D\left(\rho,(b-1) / 2,\left(a_{-}+1\right) / 2\right) \times \pi_{1}^{\prime} .
$$

D'où:

$$
\pi_{0} \hookrightarrow S D\left(\rho,(a-1) / 2,-\left(a_{-}-1\right) / 2\right) \times S D\left(\rho,(b-1) / 2,\left(a_{-}+1\right) / 2\right) \times \pi_{1}^{\prime} .
$$

Rappelons que l'induite $S D\left(\rho,(a-1) / 2,-\left(a_{-} 1\right) / 2\right) \times \pi_{0}^{\prime}$ a 2 sous-modules irréductibles (cf 5.1.1) dont l'un est $\pi_{0}$; on note l'autre $\pi_{1}$. On remarque facilement que $\pi_{1}$ est aussi un sous-module de (2). D'autre part $\left(\rho, a_{-}\right) \in \operatorname{Jord}\left(\pi_{1}^{\prime}\right)$. Donc:

$$
S D\left(\rho, a_{-}\right) \times \pi_{1}^{\prime}
$$

est irréductible. On note $\tau_{\epsilon}$, pour $\epsilon=0,1$, les 2 constituants de l'induite:

$$
S D\left(\rho, a_{-}\right) \times \pi_{0}^{\prime},
$$

de tel sorte que $\pi_{\epsilon}$ soit le sous-module de l'induite $S D\left(\rho,(a-1) / 2,\left(a_{-}+1\right) / 2\right) \times \tau_{\epsilon}$. L'induite:

$$
S D\left(\rho, a_{-}\right) \times S D\left(\rho,(b-1) / 2,\left(a_{-}+1\right) / 2\right)
$$

est exactement de longueur 2; son quotient irréductible est $S D(\rho,(b-1) / 2$, $\left.-\left(a_{-}-1\right) / 2\right)$ et elle a un sous-module que l'on note entre crochet:

$$
<S D\left(\rho, a_{-}\right) \times S D\left(\rho,(b-1) / 2,\left(a_{-}+1\right) / 2\right)>.
$$


Le module de Jacquet de $\tau_{0} \oplus \tau_{1}$ relativement au parabolique de Levi $G L\left(d_{\rho}\left(a_{-}+\right.\right.$ $\left.\left(b-a_{-}\right) / 2\right) \times G(\cdots)$ contient certainement comme quotient:

$$
<S D\left(\rho, a_{-}\right) \times S D\left(\rho,(b-1) / 2,\left(a_{-}+1\right) / 2\right)>\otimes \pi_{0}^{\prime}
$$

mais il contient aussi comme sous-quotient:

$$
S D\left(\rho,(b-1) / 2,-\left(a_{-}-1\right) / 2\right) \otimes \pi_{1}^{\prime} .
$$

On vérifie que ce terme n'intervient pas comme sous-quotient pour le module de Jacquet de l'induite

$$
<S D\left(\rho, a_{-}\right) \times S D\left(\rho,(b-1) / 2,\left(a_{-}+1\right) / 2\right)>\times \pi_{0}^{\prime} .
$$

En effet on calcule la projection du module de Jacquet sur le support cuspidale:

$$
\left.\rho\right|^{(b-1) / 2} \otimes \cdots \otimes \rho \|^{-\left(a_{-}-1\right) / 2} \otimes \cdots .
$$

Les ... de la fin signifie que l'on impose aucune restriction au support cuspidal. Les formules standard de Bernstein-Zelevinski montre que cette projection est nulle car pour l'induite considérée il y a toujours une copie (au moins) de $\rho||^{\left(a_{-}-1\right) / 2}$ qui arrive avant $\left.\rho\right|^{\left(a_{-}+1\right) / 2}$. Ainsi pour une valeur de $\epsilon$ :

$$
\tau_{\epsilon} \hookrightarrow<S D\left(\rho, a_{-}\right) \times S D\left(\rho,(b-1) / 2,\left(a_{-}+1\right) / 2\right)>\times \pi_{0}^{\prime} .
$$

Si pour l'autre valeur, la même propriété était vrai, on n'aurait pas

$$
\tau_{0} \oplus \tau_{1}=S D\left(\rho, a_{-}\right) \times \pi_{1}^{\prime} .
$$

Donc pour l'autre valeur, on a une inclusion dans:

$$
S D\left(\rho,(b-1) / 2,-\left(a_{-}-1\right) / 2\right) \times \pi_{1}^{\prime} .
$$

On en déduit maintenant que soit:

$$
\pi_{0} \hookrightarrow<S D\left(\rho,(a-1) / 2,-\left(a_{-} 1\right) / 2\right), S D\left(\rho,(b-1) / 2,\left(a_{-}+1\right) / 2\right)>\times \pi_{1}^{\prime},
$$

avec une notation que l'on espère évidente, soit

$$
\pi_{0} \hookrightarrow S D\left(\rho,(a-1) / 2,\left(a_{-}+1\right) / 2\right) \times S D\left(\rho,(b-1) / 2,-\left(a_{-}-1\right) / 2\right) \times \pi_{1}^{\prime} .
$$

Le cas (4) est favorable puisque l'induite:

$$
S D\left(\rho,(a-1) / 2,\left(a_{-}+1\right) / 2\right) \times S D\left(\rho,(b-1) / 2,-\left(a_{-}-1\right) / 2\right)
$$

est irréductible. Il entraîne qu'il existe une représentation $\pi^{\prime}$ telle que:

$$
\pi_{0} \hookrightarrow S D(\rho,(b-1) / 2,(a+1) / 2) \times \pi^{\prime},
$$

d'où

$$
\epsilon_{\pi_{0}}(\rho, b)=\epsilon_{\pi_{0}}(\rho, a) \text {. }
$$


On suppose donc que c'est $\pi_{1}$ qui vérifie l'inclusion (4) et non $\pi_{0}$. On a l'irréductibilité de l'induite $S D\left(\rho, b^{\prime}\right) \times \pi_{0}^{\prime}$ puisque $\left(\rho, b^{\prime}\right) \in \operatorname{Jord}\left(\pi_{0}^{\prime}\right)$ et donc l'induite

$S D\left(\rho,\left(a_{-}-1\right) / 2,\left(b^{\prime}+1\right) / 2\right) \times S D\left(\rho,\left(a_{-}-1\right) / 2,\left(b^{\prime}+1\right) / 2\right) \times S D\left(\rho, b^{\prime}\right) \times \pi_{0}^{\prime}$

a un unique sous-module irréductible. Il en est donc de même de l'induite:

$$
S D\left(\rho,\left(a_{-}-1\right) / 2,-\left(b^{\prime}-1\right) / 2\right) \times S D\left(\rho,\left(a_{-}-1\right) / 2,\left(b^{\prime}+1\right) / 2\right) \times \pi_{0}^{\prime} .
$$

On note $\tau_{+}$ce sous-module. La représentation:

$$
\left(S D\left(\rho,\left(a_{-}-1\right) / 2,-\left(b^{\prime}-1\right) / 2\right) \times S D\left(\rho,\left(a_{-}-1\right) / 2,\left(b^{\prime}+1\right) / 2\right)\right) \otimes \pi_{0}^{\prime}
$$

intervient dans un module de Jacquet convenable de

$$
S D\left(\rho, a_{-}\right) \times \pi_{0}^{\prime} .
$$

C'est le seul terme du semi-simplifié de ce module de Jacquet qui a une projection non nul sur le support cuspidal de ce terme. Il est donc quotient du module de Jacquet d'où $\tau_{+}$est l'un des $\tau_{\epsilon}$. Pour cette valeur de $\epsilon$, on a alors:

$$
\begin{gathered}
\pi_{\epsilon} \hookrightarrow S D\left(\rho,(a-1) / 2,\left(a_{-}+1\right) / 2\right) \times S D\left(\rho,\left(a_{-}-1\right) / 2,-\left(b^{\prime}-1\right) / 2\right) \times \\
S D\left(\rho,\left(a_{-}-1\right) / 2,\left(b^{\prime}+1\right) / 2\right) \times \pi_{0}^{\prime} .
\end{gathered}
$$

Tout sous-quotient de l'induite:

$$
\begin{gathered}
S D\left(\rho,(a-1) / 2,\left(a_{-}+1\right) / 2\right) \times S D\left(\rho,\left(a_{-}-1\right) / 2,-\left(b^{\prime}-1\right) / 2\right) \times \\
S D\left(\rho,\left(a_{-}-1\right) / 2,\left(b^{\prime}+1\right) / 2\right)
\end{gathered}
$$

est une sous-représentation d'une induite du type:

$$
S D\left(\rho,\left(a_{-}-1\right) / 2,\left(b^{\prime}+1\right) / 2\right) \times \tau^{\prime} .
$$

Il existe donc une représentation $\pi^{\prime}$ convenable et une inclusion:

$$
\pi_{\epsilon} \hookrightarrow S D\left(\rho,\left(a_{-}-1\right) / 2,\left(b^{\prime}+1\right) / 2\right) \times \pi^{\prime} .
$$

Si $\epsilon=0$ cela entraîne que:

$$
\epsilon_{\pi_{0}}\left(\rho, a_{-}\right)=\epsilon_{\pi_{0}}\left(\rho, b^{\prime}\right) .
$$

Et on aurait aussi terminé. On suppose encore qu'il n'en est pas ainsi. Si l'on savait que $\pi_{1}$ est une aussi une série discrète, on aurait (5) et (6) pour $\pi_{1}$ ce qui est impossible pour le raisonnement fait pour $\pi_{0}$ (il n'y a plus de différence entre $\pi_{0}$ et $\pi_{1}$ ).

Il est vrai que $\pi_{1}$ est une série discrète (cela sera montré en [M-T]) mais puisque ce n'est pas démontré ici, il vaut mieux procéder différemment.

En étant plus précis quand on construit $\tau_{+}$, on montre que $\tau_{+}$est l'unique sous-module de l'induite:

$$
S D\left(\rho,(a-1) / 2,-\left(b^{\prime}-1\right) / 2\right) \times S D\left(\rho,\left(a_{-}-1\right) / 2,\left(b^{\prime}-1\right) / 2\right) \times \pi_{0}^{\prime} .
$$


En particulier l'un de ses modules de Jacquet contient un terme du type:

$$
\begin{gathered}
\rho||^{(a-1) / 2} \otimes \cdots \otimes \rho||^{\left(a_{-}+1\right) / 2} \\
\otimes \rho||^{\left(a_{-}-1\right) / 2} \otimes \rho||^{\left(a_{-}-1\right) / 2} \otimes \cdots \otimes \rho||^{\left(b^{\prime}+1\right) / 2} \times \|\left.\right|^{\left(b^{\prime}+1\right) / 2} \otimes \lambda,
\end{gathered}
$$

où $\lambda$ est une représentation quelconque. Puisque $\tau_{+}$a été supposé être $\pi_{1}$ et vérifier l'inclusion (4) un tel terme doit apparaître dans le module de Jacquet de l'induite:

$$
S D\left(\rho,(b-1) / 2,-\left(a_{-}-1\right) / 2\right) \times S D\left(\rho,(a-1) / 2,\left(a_{-}+1\right) / 2\right) \times \pi_{1}^{\prime} .
$$

Pour que ceci soit possible il faut assurément qu'il existe un ensemble $Z$ de demientiers inclus dans l'ensemble $\left[(a-1) / 2,\left(b^{\prime}+1\right) / 2\right]$ (totalement ordonné par les valeurs décroissantes) contenant l'ensemble $\left[(a-1) / 2,\left(b^{\prime}+1\right) / 2\right]$ (il peut y avoir multiplicité 2 pour les éléments de ce dernier ensemble) tel qu'il existe une représentation $\pi^{\prime}$ et une inclusion:

$$
\pi_{1}^{\prime} \hookrightarrow \times_{z \in Z} \rho||^{z} \times \pi^{\prime} .
$$

Soit $z_{0}$ l'élément de plus grande valeur de $Z$. Un tel $z_{0}$ est nécessairement de la forme $\left(a^{\prime}-1\right) / 2$ avec $\left(\rho, a^{\prime}\right) \in \operatorname{Jord}\left(\pi_{1}^{\prime}\right)$. Les conditions sur $z$ entrainent alors que

$$
a^{\prime} \in\left[a_{-}, a\right] .
$$

La seule valeur de $a^{\prime}$ convenant est exactement $a_{-}$; c'est le bloc de Jordan $(\rho, b)$ de $\pi_{0}^{\prime}$ qui s'est transformé en $\left(\rho, a_{-}\right)$quand on est passé de $\pi_{0}^{\prime}$ à $\pi_{1}^{\prime}$. Il faut donc l'existence d'une représentation $\pi^{\prime}$ telle que:

$$
\left.\pi_{1}^{\prime} \hookrightarrow \rho\right|^{\left(a_{-}-1\right) / 2} \times \pi^{\prime} .
$$

Si $a_{-}=b^{\prime}+2$ cela contredit

$$
\epsilon_{\pi_{0}^{\prime}}(\rho, b) \neq \epsilon_{\pi_{0}^{\prime}}\left(\rho, b^{\prime}\right)
$$

Si par contre $b^{\prime}+2<a_{-}, \pi^{\prime}$ existe et est nécessairement une série discrète, mais $Z$ n'est alors par réduit à $z_{0}$ car $Z$ contient $\left[\left(a_{-}-1\right) / 2,\left(b^{\prime}+1\right) / 2\right]$. De proche en proche, on montre qu'il existe une représentation $\pi^{\prime \prime}$ et une inclusion:

$$
\pi_{1}^{\prime} \hookrightarrow \rho||^{\left(a_{-}-1\right) / 2} \times \cdots \times \rho||^{\left(b^{\prime}+1\right) / 2} \times \pi^{\prime \prime} .
$$

et maintenant on a:

$$
\pi_{0}^{\prime} \hookrightarrow \times \rho||^{(b-1) / 2} \times \cdots \times \rho||^{\left(b^{\prime}+1\right) / 2} \times \pi^{\prime \prime},
$$

ce qui contredit:

$$
\epsilon_{\pi_{0}^{\prime}}(\rho, b) \neq \epsilon_{\pi_{0}^{\prime}}\left(\rho, b^{\prime}\right)
$$

et termine la démonstration. 
5.5 Lemme: On reprend les hypothèses du lemme 5.4, c'est-à-dire:

$$
\pi_{0} \hookrightarrow S D\left(\rho,(a-1) / 2,-\left(a_{-}-1\right) / 2\right) \times \pi_{0}^{\prime} .
$$

Soit ici $b \in \mathbf{N}$ tel que $(\rho, b) \in \operatorname{Jord}\left(\pi_{0}^{\prime}\right)$ et on suppose que b est paire. Alors:

$$
\epsilon_{\pi_{0}}(\rho, b)=\epsilon_{\pi_{0}^{\prime}}(\rho, b) .
$$

Etant donné le lemme précédent 5.4 et les définitions, il suffit de démontrer l'assertion quand $b$ est minimal parmi les entiers vérifiant $(\rho, b) \in \operatorname{Jord}\left(\pi_{0}^{\prime}\right)$. Ce cas se montre comme le lemme 4.1 lui-même en posant $b^{\prime}=0$ et $\epsilon_{\tilde{\pi_{0}}}(\rho, 0)=1$ pour toute série discrète $\tilde{\pi}_{0}$.

\section{Première extension de la définition de $\epsilon_{\pi_{0}}$}

6.0 Soit $\pi_{0}$ une série discrète et soit $(\rho, a) \in \operatorname{Jord}\left(\pi_{0}\right)$. On a défini $\epsilon_{\pi_{0}}(\rho, a)$ si $a$ est paire. Dans le cas inverse, on ne connait que les différences:

$$
\epsilon_{\pi_{0}}(\rho, a) \epsilon_{\pi_{0}}\left(\rho, a_{-}\right)^{-1}
$$

quand $a_{-}$est défini. Rappelons que grâce au paragraphe 4, on sait qu'il existe des triplets $\left(\rho^{\prime}, x^{\prime}, y^{\prime}\right)$ et une représentation cuspidale, $\pi_{\text {cusp }}$ d'un groupe $G\left(n^{\prime}, F\right)$ avec $n^{\prime} \leq n$ tels que:

$$
\pi_{0} \hookrightarrow \times{ }_{\left(\rho^{\prime}, x^{\prime}, y^{\prime}\right)} S D\left(\rho^{\prime}, x^{\prime}, y^{\prime}\right) \times \pi_{c u s p} .
$$

On sait (c'est la notion de support cuspidal) que $\pi_{c u s p}$ est uniquement déterminé par $\pi_{0}$. On appellera cette représentation le support cuspidal partiel de $\pi_{0}$; ce n'est pas le support cuspidal puisque l'on a oublié le support cuspidal des $S D\left(\rho^{\prime}, x^{\prime}, y^{\prime}\right)$. Remarquons que la donnée de $\operatorname{Jord}\left(\pi_{0}\right)$ et de $\pi_{c u s p}$ permet de retrouver la totalité du support cuspidal. D'où la raison de notre oubli.

On va définir $\epsilon_{\pi_{0}}(\rho, a)$ pour $(\rho, a) \in \operatorname{Jord}\left(\pi_{0}\right)$ et $a$ impair sous l'hypothèse que $(\rho, 1) \notin \operatorname{Jord}\left(\pi_{c u s p}\right)$. Ceci est fait par la proposition ci-dessous qui demande quelques préambules.

L'hypothèse entraîne que pour tout entier $a^{\prime},\left(\rho, a^{\prime}\right) \notin \operatorname{Jord}\left(\pi_{c u s p}\right)$ (cf le début de 4). Ainsi pour tout $a^{\prime} \in \mathbf{N}$ impair:

$$
S D\left(\rho, a^{\prime}\right) \times \pi_{c u s p}
$$

est semi-simple de longueur 2. On note $\tau_{\epsilon}\left(a^{\prime}\right)$ pour $\epsilon= \pm 1$ les 2 composantes. Quelque soit $\epsilon= \pm 1$ et pour tout $b \in \mathbf{N}$ :

$$
(\rho, b) \in \operatorname{Jord}\left(\tau_{\epsilon}\left(a^{\prime}\right)\right) \Leftrightarrow b=a^{\prime} .
$$

On peut donc faire un choix de $\epsilon_{\tau_{\epsilon}\left(a^{\prime}\right)}\left(\rho, a^{\prime}\right)$ de façon à séparer les deux représentations. Dans l'état actuel, je ne connais pas de façon canonique de le faire, mais on sait que l'opérateur d'entrelacement standard de cette induite agit par des scalaires différents sur chacun des morceaux; évidemment ces scalaires ne sont pas \pm 1 pour qu'ils le deviennent, il faut normaliser. 
Fixons donc une normalisation des opérateurs d'entrelacement; en [M1], on l'a fait à une fonction holomorphe inversible près de plusieurs façons, les différentes façons se distinguant par la présence de zéro ou non dans le domaine positif. Pour le moment prenons n'importe laquelle de ces normalisation (mais la définition dépend de ce choix et on fixe la fonction inversible (qui traîne dans tout [M1]) de tel sorte que la formule de produit:

$$
\rho^{\prime}||^{s} \times \pi \rightarrow\left(\rho^{\prime}\right)^{*}||^{-s} \times \pi \rightarrow \rho^{\prime}||^{s} \times \pi
$$

donne 1, pour tout $\pi$ cuspidal d'un $G\left(n^{\prime}, F\right)$ et tout $\rho^{\prime}$ cuspidale d'un groupe linéaire $G L\left(d_{\rho^{\prime}}, F\right)$. L'existence d'un tel choix est un argument formel déjà utilisé il y a plus de 15 ans par Arthur [A1]. On fixe aussi une normalisation pour les opérateurs d'entrelacement entre induites de cuspidales pour les groupes linéaires de tel sorte que pour tout $\rho^{\prime}$ cuspidale unitaire ou non, l'entrelacement:

$$
\rho^{\prime}||^{s} \times \rho^{\prime} \rightarrow \rho^{\prime} \times \rho^{\prime} \|^{s}
$$

soit l'identité en $s=0$. Cela permet de définir une normalisation pour les opérateurs:

$$
S D\left(\rho^{\prime}, a^{\prime}\right)||^{s} \times \pi \rightarrow S D\left(\rho^{\prime}, a^{\prime}\right)||^{-s} \times \pi,
$$

(ici $\rho^{\prime}$ est autoduale) quand $\pi$ est une série discrète (ou même une représentation quelconque): on plonge $\pi$ dans une induite de cuspidale et $S D\left(\rho^{\prime}, a^{\prime}\right)$ dans $\times_{k \in[(a-1) / 2,-(a-1) / 2]} \rho^{\prime}||^{k}$. En $s=0$ l'opérateur d'entrelacement est indépendant du plongement (on le vérifie grâce aux propriétés du support cuspidal). On vérifie que si $\pi$ est une série discrète, par unitarité, l'opérateur ainsi défini est holomorphe en $s=0$.

Revenons à $\pi_{0}$ et $\rho$ satisfaisant les hypothèses du début du paragraphe. Soit $a \in \mathbf{N}$ tel que $(\rho, a) \in \operatorname{Jord}\left(\pi_{0}\right)$ et $a_{-}$est défini et vérifie:

$$
\epsilon_{\pi_{0}}(\rho, a)=\epsilon_{\pi_{0}}\left(\rho, a_{-}\right)
$$

il existe au moins un tel choix de $a$. L'induite:

$$
S D\left(\rho, a_{-}\right) \times \pi_{0}
$$

est irréductible et l'opérateur d'entrelacement normalisé est un scalaire en $s=0$; on note $\lambda_{\pi_{0}}\left(\rho, a_{-}\right)$ce scalaire. Après avoir fait un tel choix, on définit:

$$
\epsilon_{\pi_{0}}(\rho, a)=\epsilon_{\pi_{0}}\left(\rho, a_{-}\right)=\lambda_{\pi_{0}}\left(\rho, a_{-}\right) .
$$

On démontre:

6.1 Proposition: (i) La définition de $\epsilon_{\pi_{0}}\left(\rho, a^{\prime}\right)$ pour tout $a^{\prime} \in \mathbf{N}$ tel que $\left(\rho, a^{\prime}\right) \in$ $\operatorname{Jord}\left(\pi_{0}\right)$ qui découle du choix ci-dessus est indépendante du choix de a fait. Pour tout $(\rho, b) \in \operatorname{Jord}\left(\pi_{0}\right), \epsilon_{\pi_{0}}(\rho, b)$ est donc le scalaire par lequel agit l'opérateur d'entrelacement normalisé en $s=0$ de l'induite:

$$
S D(\rho, b)||^{s} \times \pi_{0} .
$$


(ii) Soit $\left(\rho^{\prime}, a^{\prime}\right) \in \operatorname{Jord}_{\pi_{0}}$ tel que $a_{-}^{\prime}$ soit défini avec $\epsilon_{\pi_{0}}\left(\rho^{\prime}, a^{\prime}\right)=\epsilon_{\pi_{0}}\left(\rho^{\prime}, a_{-}^{\prime}\right)$. Et soit $\pi_{0}^{\prime}$ la série discrète telle que:

$$
\pi_{0} \hookrightarrow S D\left(\rho^{\prime},\left(a^{\prime}-1\right) / 2,-\left(a_{-}^{\prime}-1\right) / 2\right) \times \pi_{0}^{\prime} .
$$

Et soit $b \in \mathbf{N}$ tel que $(\rho, b) \in \operatorname{Jord}\left(\pi_{0}^{\prime}\right)$. Alors: $\epsilon_{\pi_{0}^{\prime}}(\rho, b)$ (que l'on suppose défini par récurrence) est égal à $\epsilon_{\pi_{0}}(\rho, b)$.

Avant de démontrer cette proposition, on donne d'autres définitions de $\lambda_{\pi_{0}}\left(\rho, a_{-}\right)$.

6.1.1 Première réinterprétation de $\lambda_{\pi_{0}}\left(\rho, a_{-}\right)$. Avec les hypothèses sur $\pi_{0}$ et $a, a_{-}$, on note $\pi_{1}$ la série discrète telle que:

$$
\pi_{0} \hookrightarrow S D\left(\rho,(a-1) / 2,-\left(a_{-}-1\right) / 2\right) \times \pi_{1} .
$$

On a une inclusion:

$S D\left(\rho,(a-1) / 2,-\left(a_{-}-1\right) / 2\right) \hookrightarrow S D\left(\rho,(a-1) / 2,\left(a_{-}+1\right) / 2\right) \times S D\left(\rho, a_{-}\right)$.

Sur l'induite:

$$
S D\left(\rho,(a-1) / 2,\left(a_{-}+1\right) / 2\right) \times\left. S D\left(\rho, a_{-}\right)\right|^{s} \times \pi_{1}
$$

il y a un opérateur d'entrelacement. En $s=0$ cet opérateur d'entrelacement est scalaire sur chacun des 2 sous-modules de l'induite (2). On va montrer que $\lambda_{\pi_{0}}\left(\rho, a_{-}\right)$n'est autre que le scalaire par lequel il agit sur $\pi_{0}$. Pour la démonstration, on note $\lambda_{\pi_{0}}^{\prime}\left(\rho, a_{-}\right)$ce scalaire. On regarde l'induite:

$$
S D\left(\rho, a_{-}\right)||^{s^{\prime}} \times S D\left(\rho,(a-1) / 2,-\left(a_{-}-1\right) / 2\right) \times \pi_{1} .
$$

Pour tout $s^{\prime}$ voisin de 0 , elle est isomorphe à l'induite:

$$
S D\left(\rho,(a-1) / 2,-\left(a_{-}-1\right) / 2\right) \times S D\left(\rho, a_{-}\right)||^{s^{\prime}} \times \pi_{1} .
$$

Ainsi $S D\left(\rho, a_{-}\right) \times \pi_{0}$ est un sous-module de l'induite ci-dessus et $\lambda_{\pi_{0}}\left(\rho, a_{-}\right)$est le scalaire par lequel opère l'opérateur d'entrelacement normalisé (qui change $s^{\prime}$ en $-s^{\prime}$ ) défini sur l'induite ci-dessus et restreint à ce sous-module. On plonge encore cette induite dans l'induite ci-dessous en $s=0$ :

$$
S D\left(\rho,(a-1) / 2,\left(a_{-}+1\right) / 2\right) \times\left. S D\left(\rho, a_{-}\right)\right|^{s} \times S D\left(\rho, a_{-}\right)||^{s^{\prime}} \times \pi_{1} .
$$

Comme les opérateurs d'entrelacement pour $\eta \in\{ \pm 1\}$ et $\eta^{\prime} \in \pm 1$ :

$$
S D\left(\rho, a_{-}\right)||^{\eta s} \times S D\left(\rho, a_{-}\right)||^{\eta^{\prime} s^{\prime}} \rightarrow S D\left(\rho, a_{-}\right)||^{\eta^{\prime} s^{\prime}} \times S D\left(\rho, a_{-}\right)||^{\eta s}
$$

sont des bijections qui valent l'identité en $s=s^{\prime}=0$, on en déduit l'égalité cherchée:

$$
\lambda_{\pi_{0}}\left(\rho, a_{-}\right)=\lambda_{\pi_{0}}^{\prime}\left(\rho, a_{-}\right)
$$


6.1.2 Deuxième réinterprétation de $\lambda_{\pi_{0}}\left(\rho, a_{-}\right)$. On donne encore une autre définition de $\lambda_{\pi_{0}}\left(\rho, a_{-}\right)$avec les hypothèses où nous l'avons défini: c'est le scalaire par lequel agit l'opérateur d'entrelacement en $s=0$ de l'induite:

$$
S D(\rho, a)||^{s} \times \pi_{0} \rightarrow S D(\rho, a)||^{-s} \times \pi_{0} .
$$

On note $N_{a}(s)$ l'opérateur d'entrelacement normalisé:

$$
S D(\rho, a)||^{s} \times \pi_{0}^{\prime} \rightarrow S D(\rho, a)||^{-s} \times \pi_{0}^{\prime} .
$$

On définit de même $N_{a_{-}}(s)$ en remplaçant $a$ par $a_{-}$dans la définition ci-dessus. On va calculer $N_{a}(0)$ en fonction de $N_{a_{-}}(0)$. Pour cela, on note $R(s)$ l'opérateur d'entrelacement normalisé:

$$
\begin{gathered}
S D\left(\rho,(a-1) / 2,\left(a_{-}+1\right) / 2\right)||^{-s} \times S D\left(\rho,(a-1) / 2,-\left(a_{-}-1\right) / 2\right)||^{s} \times \pi_{0}^{\prime} \\
\downarrow \\
\left.S D\left(\rho,(a-1) / 2,-\left(a_{-}-1\right) / 2\right)\right|^{s} \times S D\left(\rho,-\left(a_{-}+1\right) / 2,-(a-1) / 2\right)||^{s} \times \pi_{0}^{\prime} .
\end{gathered}
$$

Cet opérateur est au moins méromorphiquement défini. Vérifions tout de suite qu'il est holomorphiquement défini et bijectif en $s=0$. Cet opérateur se décompose encore en le produit de 2 opérateurs: l'un faisant intervenir les groupes linéaires et le résultat est connu pour cet opérateur et l'autre est simplement:

$S D\left(\rho,(a-1) / 2,\left(a_{-}+1\right) / 2\right)||^{-s} \times \pi_{0}^{\prime} \rightarrow S D\left(\rho,-\left(a_{-}+1\right) / 2,-(a-1) / 2\right)||^{s} \times \pi_{0}^{\prime}$.

En $s=0$ cette induite est irréductible car pour tout $(\rho, b) \in \operatorname{Jord}\left(\pi_{0}^{\prime}\right)$, on a:

$$
(b+1) / 2 \notin\left[(a-1) / 2,\left(a_{-}+1\right) / 2\right] .
$$

Cela suffit par 5.4.1. L'opérateur considéré est le produit de l'opérateur d'entrelacement standard par un facteur de normalisation que nous notons $r_{+}(-s)^{-1}$. On n'a volontairement pas précisé quelle normalisation précise nous prenons parce que toutes celles considérées en [M1] conviennent. La plus intéressante à prendre (cf. la remarque ci-dessous) est celle notée $N_{A}(\cdots)$ en [M1], c'est-à-dire celle qui dans le domaine positif a le plus de zéros. Pour le lecteur, nous récrivons la valeur de $r_{+}(-s)$ pour cette normalisation:

$$
\begin{gathered}
\prod_{k \in\left[(a-1) / 2,\left(a_{-}+1\right) / 2\right]} \prod_{\left(\rho^{\prime}, a^{\prime}\right) \in \operatorname{Jord}\left(\pi_{0}^{\prime}\right)} L\left(\rho \times \rho^{\prime},-s+k-\left(a^{\prime}-1\right) / 2\right) \times \\
L\left(\rho \times \rho^{\prime},-s+k+\left(a^{\prime}+1\right) / 2\right)^{-1} \times m(s),
\end{gathered}
$$

où $m(s)$ est un quotient de fonctions $L(\rho, R, b(s))$ qui n'a ni pôle ni zéro dans notre situation. Nous utilisons encore ici le fait que $\operatorname{Jor} d\left(\pi_{0}^{\prime}\right)$ ne contient pas d'éléments de la forme $(\rho, b)$ avec $b \in\left[a, a_{-}\right]$pour vérifier qu'en $s=0, r_{+}^{\prime}(-s)$ est holomorphe inversible. L'opérateur d'entrelacement standard est lui aussi holomorphe en $s=0$, bijectif par irréductibilité. Cela prouve que $R(0)$ est bien, comme annoncé holomorphe bijectif. 
On calcule le composé:

$$
\begin{gathered}
R(s)^{-1} \circ N_{a}(s) \circ R(-s): \\
S D\left(\rho,(a-1) / 2,\left(a_{-}+1\right) / 2\right)||^{-s} \times\left. S D\left(\rho,(a-1) / 2,-\left(a_{-}-1\right) / 2\right)||^{s}\right|^{s} \times \pi_{0}^{\prime} \\
\downarrow \\
S D\left(\rho,(a-1) / 2,\left(a_{-}+1\right) / 2\right)||^{s} \times S D\left(\rho,(a-1) / 2,\left(a_{-}+1\right) / 2\right)||^{-s} \times \\
S D\left(\rho, a_{-}\right)||^{-s} \times \pi_{0}^{\prime} .
\end{gathered}
$$

En utilisant les formules de produit, on voit que cet opérateur est la restriction à la sous-représentation écrite, du produit des 2 opérateurs d'entrelacement agissant sur:

$$
\begin{gathered}
S D\left(\rho,(a-1) / 2,\left(a_{-}+1\right) / 2\right)||^{-s} \times S D\left(\rho,(a-1) / 2,\left(a_{-}+1\right) / 2\right)||^{s} \times \\
S D\left(\rho, a_{-}\right)||^{s} \times \pi_{0}^{\prime}
\end{gathered}
$$

le premier échangeant les 2 premiers facteurs et le deuxième n'étant autre que $N_{a_{-}}(s)$. En $s=0$ l'échange des 2 premiers facteurs est l'identité (grâce aux normalisations), d'où:

$$
R(0)^{-1} \circ N_{a}(0) \circ R(0)=N_{a_{-}}(0) .
$$

Cette formule s'interprète en terme de représentations: les induites $S D(\rho, a) \times \pi_{0}^{\prime}$ et $S D\left(\rho, a_{-}\right) \times \pi_{0}^{\prime}$ sont exactement de longueur 2 ; on note $\tau_{\epsilon}$ et $\tau_{\epsilon}^{-}$pour $\epsilon= \pm 1$ les deux sous-modules respectivement où $\epsilon$ est la valeur de l'opérateur d'entrelacement sur le sous-module. Alors, on vient de montrer, pour $\epsilon= \pm 1$ :

$$
\tau_{\epsilon} \hookrightarrow S D\left(\rho,(a-1) / 2,\left(a_{-}+1\right) / 2\right) \times S D\left(\rho,(a-1) / 2,\left(a_{-}+1\right) / 2\right) \times \tau_{\epsilon}^{-} .
$$

On montre maintenant l'assertion:

$$
\lambda_{\pi_{0}}(\rho, a)=\lambda_{\pi_{0}}\left(\rho, a_{-}\right)
$$

sous les hypothèses faites. On remarque que: $S D(\rho, a) \times \pi_{0}^{\prime}$ et $S D(\rho,(a-1) / 2$, $\left.\left(a_{-}+1\right) / 2\right) \times \pi_{0}$ ont une sous-représentation irréductible commune. C'est même l'unique sous-représentation de la deuxième induite écrite; pour la première induite, c'est l'unique sous-module de l'induite (avec les notations ci-dessus):

$$
S D\left(\rho,(a-1) / 2,\left(a_{-}+1\right) / 2\right) \times S D\left(\rho,(a-1) / 2,\left(a_{-}+1\right) / 2\right) \times \tau_{\lambda_{\pi_{0}}}\left(\rho, a_{-}\right) .
$$

Ainsi $\lambda_{\pi_{0}}(\rho, a)$ n'est autre que la restriction à cette sous-représentation de l'opérateur en $s=0$ :

$$
S D(\rho, a)||^{s} \times S D(\rho, a) \times \pi_{0}^{\prime} .
$$

Cela se calcule aussi en échangeant les 2 copies de $S D(\rho, a)$ et $\lambda_{\pi_{0}}(\rho, a)$ est la valeur de l'opérateur d'entrelacement en $s=0$ :

$$
S D(\rho, a)||^{s} \times \pi_{0}^{\prime}
$$

sur le sous-module (1). On a précisément démontré que cela valait $\lambda_{\pi_{0}}\left(\rho, a_{-}\right)$. 
6.1.3 Remarque (cette remarque nécessite que les hypothèses sur les conjectures d'Arthur de [M1] soient satisfaites): Avec la normalisation à la Arthur comme expliquée en [M1] et en supposant $\pi$ cuspidal, on note $\lambda_{\pi}(\rho, a)$ la valeur en $s=0$ de l'opérateur d'entrelacement:

$$
\left.S D(\rho, a)\right|^{S} \times \pi,
$$

pour $(\rho, a) \in \operatorname{Jord}(\pi)$. Cela suppose évidemment un certain nombre de choix comme une identification de $\rho$ avec $\rho^{*}$, que l'on fait indépendamment de $a$. Alors, pour $(\rho, a)$ et $(\rho, b)$ dans $\operatorname{Jord}(\pi)$ :

$$
\lambda_{\pi}(\rho, a) \lambda_{\pi}(\rho, b)^{-1}=(-1)^{(a-b) / 2} .
$$

Nous n'utiliserons pas cette remarque parce que nous ne voulons faire que l'hypothèse de l'introduction et c'est la raison pour laquelle nous ne décrivons pas plus précisément ce qu'est la normalisation à la Arthur, disons simplement que c'est, parmi les normalisations attachées à chaque paquet d'Arthur contenant conjecturalement $\pi_{0}$, celle qui a le plus de zéros dans le domaine positif. Toutefois une définition de ce genre du caractère $\epsilon_{\pi_{0}}$ simplifierait grandement le paragraphe 5 . On suppose que $a>b$. La différence avec la démonstration ci-dessus est que $R(s)$ est ici d'ordre $(a-b) / 2$ en $s=0$. D'où le signe.

Avec cela, on peut démontrer, comme on va le faire ci-dessous dans le cas particulier qui nous intéresse, que, pour tout $\pi_{0}$ série discrète et pour tout $(\rho, a)$, $(\rho, b)$ dans $\operatorname{Jord}\left(\pi_{0}\right)$, on a:

$$
\lambda_{\pi_{0}}(\rho, a) \lambda_{\pi_{0}}(\rho, b)^{-1}=\epsilon_{\pi_{0}}(\rho, a) \epsilon_{\pi_{0}}(\rho, b)^{-1} .
$$

Mais nous ne le faisons pas, car nous n'avons pour le moment aucun moyen de fixer, de façon raisonable la normalisation des opérateurs ( $\rho$ autodual unitaire):

$$
\rho||^{s} \times \pi \rightarrow \rho||^{-s} \times \pi
$$

quand les induites sont irréductibles en $s=0$. On voudrait un choix qui ne dépende pas de $\rho$ tel que $(\rho, 1) \in \operatorname{Jord}(\pi)$ ni de $\pi$ dans son paquet et qui ne soit pas identiquement 1 .

6.1.4 Démonstration de la proposition 6.1. On démontre maintenant la proposition dont on reprend les notations. On fixe donc un choix de $a$ pour définir $\epsilon_{\pi_{0}}(\rho$, ?). Pour tout $b \in \mathbf{N}$ tel que $(\rho, b) \in \operatorname{Jord}\left(\pi_{0}\right)$, on note $\lambda_{\pi_{0}}(\rho, b)$ le scalaire par lequel opère l'opérateur d'entrelacement, en $s=0$, de l'induite:

$$
S D(\rho, b)||^{s} \times \pi_{0} .
$$

Il suffit de démontrer que si $b$ est tel que $b_{-}$est défini, alors:

$$
\lambda_{\pi_{0}}(\rho, b)=\lambda_{\pi_{0}}\left(\rho, b_{-}\right) \Leftrightarrow
$$

il existe une série discrète $\pi_{1}$ et une inclusion:

$$
\pi_{0} \hookrightarrow S D\left(\rho,(b-1) / 2,\left(b_{-}+1\right) / 2 \times S D\left(\rho, b_{-}\right) \times \pi_{1} .\right.
$$


On démontre cette propriété par récurrence sur $n$; on reprend l'inclusion:

$$
\pi_{0} \hookrightarrow S D\left(\rho,(a-1) / 2,-\left(a_{-}-1\right) / 2\right) \times \pi_{0}^{\prime} .
$$

On fixe $b$ comme ci-dessus. Si $b=a$, on a déjà vérifié que $\lambda_{\pi_{0}}(\rho, a)=\lambda_{\pi_{0}}\left(\rho, a_{-}\right)$. Si $b_{-}>a$ ou si $b<a_{-}$, on a l'assertion par récurrence: en effet les induites $S D(\rho, b) \times S D\left(\rho,(a-1) / 2,-\left(a_{-}-1\right) / 2\right)$ et $S D\left(\rho, b_{-}\right) \times S D(\rho,(a-1) / 2$, $-\left(a_{-}-1\right) / 2$ ) sont irréductibles (le segment qui définit chacun des 2 termes vérifie une propriété d'inclusion). On a donc sûrement:

$$
\lambda_{\pi_{0}}(\rho, b)=\lambda_{\pi_{0}}\left(\rho, b_{-}\right) \Leftrightarrow \lambda_{\pi_{0}^{\prime}}(\rho, b)=\lambda_{\pi_{0}^{\prime}}\left(\rho, b_{-}\right) .
$$

Comme on a démontré en 5.4 la même équivalence pour l'autre terme de l'équivalence cherchée, on conclut par récurrence.

Il reste les 2 cas, $b_{-}=a$ et $b=a_{-}$. Traitons d'abord le cas, où $b=a_{-}$. On sait que l'induite:

$$
S D\left(\rho,(a-1) / 2,-\left(a_{-}-1\right) / 2\right) \times \pi_{0}^{\prime}
$$

a exactement 2 sous-modules dont l'un est $\pi_{0}$; on note $\pi_{1}$ l'autre sous-module. Sans savoir que $\pi_{1}$ est une série discrète, on peut définir $\lambda_{\pi_{1}}\left(\rho, b^{\prime}\right)$ pour tout $\left(\rho, b^{\prime}\right) \in \operatorname{Jord}\left(\pi_{0}\right)$ (de fait $\pi_{1}$ doit être une série discrète avec les mêmes blocs de Jordan que $\left.\pi_{0}\right)$. On utilise la première définition de $\lambda_{\pi_{0}}\left(\rho, a_{-}\right)$donné ci-dessus, c'est-à-dire comme le scalaire pour l'action de l'opérateur d'entrelacement en $s=0$ pour l'induite:

$$
S D\left(\rho,(a-1) / 2,\left(a_{-}+1\right) / 2\right) \times S D\left(\rho, a_{-}\right)||^{s} \times \pi_{0}^{\prime} .
$$

Puisque les opérateurs d'entrelacement séparent les sous-modules de $S D\left(\rho, a_{-}\right)||^{s}$ $\times \pi_{0}^{\prime}$ en $s=0$, on sait que:

$$
\lambda_{\pi_{0}}\left(\rho, a_{-}\right) \neq \lambda_{\pi_{1}}\left(\rho, a_{-}\right) .
$$

Il y a exactement 1 sous-module de $S D\left(\rho, a_{-}\right) \times \pi_{0}^{\prime}$ qui soit aussi sous-module de l'induite:

$S D\left(\rho,\left(a_{-}-1\right) / 2,\left(b_{-}+1\right) / 2\right) \times S D\left(\rho,\left(a_{-}-1\right) / 2,\left(b_{-}+1\right) / 2\right) \times S D\left(\rho, b_{-}\right) \times \pi_{0}^{\prime} ;$

cela se voit sur le module de Jacquet. Il y a donc exactement une valeur de $\epsilon$ telle que:

$$
\begin{gathered}
\pi_{\epsilon} \hookrightarrow S D\left(\rho(a-1) / 2,\left(a_{-}+1\right) / 2\right) \times S D\left(\rho,\left(a_{-}-1\right) / 2,\left(b_{-}+1\right) / 2\right) \times \\
S D\left(\rho,\left(a_{-}-1\right) / 2,\left(b_{-}+1\right) / 2\right) \times S D\left(\rho, b_{-}\right) \times \pi_{0}^{\prime} .
\end{gathered}
$$

Pour cette valeur de $\epsilon$, on a donc:

$$
\lambda_{\pi_{\epsilon}}\left(\rho, a_{-}\right)=\lambda_{\pi_{\epsilon}}\left(\rho, b_{-}\right) .
$$

Et donc pour l'autre, l'inégalité. En reprenant l'inclusion ci-dessus, on voit que pour cette valeur de $\epsilon$, il existe une représentation $\pi^{\prime}$ et une inclusion:

$$
\pi_{\epsilon} \hookrightarrow S D\left(\rho,\left(a_{-}-1\right) / 2,\left(b_{-}+1\right) / 2\right) \times \pi^{\prime} .
$$


On vient donc de démontrer l'implication:

$$
\epsilon_{\pi_{0}}\left(\rho, a_{-}\right) \neq \epsilon_{\pi_{0}}\left(\rho, b_{-}\right) \Rightarrow \lambda_{\pi_{0}}\left(\rho, a_{-}\right) \neq \lambda_{\pi_{0}}\left(\rho, b_{-}\right) .
$$

Pour terminer ce cas, il suffit de remarquer que si $\epsilon_{\pi_{0}}\left(\rho, a_{-}\right)$vaut $\epsilon_{\pi_{0}}\left(\rho, b_{-}\right)$, on a déjà démontré l'égalité analogue pour les $\lambda_{\pi_{0}}$.

Traitons le cas $b_{-}=a$. L'hypothèse sur $\pi_{\text {cusp }}$ est ici utile; elle entraîne que pour $\pi_{0}^{\prime}$, il existe un couple $\left(b^{\prime}, b^{\prime \prime}\right)$ de deux entiers tels que $\left(\rho, b^{\prime}\right),\left(\rho, b^{\prime \prime}\right) \in \operatorname{Jord}\left(\pi_{0}^{\prime}\right)$ consécutif pour cette propriété et tel que

$$
\epsilon_{\pi_{0}^{\prime}}\left(\rho, b^{\prime}\right)=\epsilon_{\pi_{0}^{\prime}}\left(\rho, b^{\prime \prime}\right) .
$$

On fixe un tel couple; avec l'hypothèse $b_{-}=a$, si $b \notin\left\{b^{\prime}, b^{\prime \prime}\right\}$ on a sûrement soit $b^{\prime \prime}>b$ soit $b^{\prime}<a_{-}$. On a alors $b^{\prime \prime}=b_{-}^{\prime}$ et il est très facile d'obtenir le résultat cherché par récurrence. Si $b^{\prime \prime}=b$, on a encore $b^{\prime \prime}=b_{-}^{\prime}$ et on obtient le résultat par le cas traité ci-dessus en remplaçant le rôle de $\left(a, a_{-}\right)$par celui de $\left(b^{\prime}, b_{-}^{\prime \prime}=b\right)$. Reste donc le cas $b>a>a_{-}>b^{\prime \prime}$. Les arguments sont toujours du même type; on reprend la notation $\pi_{\epsilon}$ introduite ci-dessus et on vérifie ici qu'il y a exactement 1 valeur de $\epsilon$ pour laquelle:

$$
\begin{gathered}
\pi_{\epsilon} \hookrightarrow S D\left(\rho,(a-1) / 2,\left(a_{-}+1\right) / 2 \times S D\left(\rho,(b-1) / 2,\left(b^{\prime \prime}+1\right) / 2\right) \times\right. \\
S D\left(\rho, a_{-}\right) \times S D\left(\rho, b^{\prime \prime}\right) \times \pi_{1}^{\prime},
\end{gathered}
$$

pour $\pi_{1}^{\prime}$ une série discrète convenable. Pour cette valeur de $\epsilon$ :

$$
\lambda_{\pi_{\epsilon}}\left(\rho, a_{-}\right)=\lambda_{\pi_{\epsilon}}\left(\rho, b^{\prime \prime}\right)
$$

alors que pour l'autre, on a l'inégalité. En outre pour cette valeur de $\epsilon$ l'inclusion ci-dessus et l'irréductibilité de

$$
S D\left(\rho,(a-1) / 2,\left(a_{-}+1\right) / 2 \times S D\left(\rho,(b-1) / 2,\left(b^{\prime \prime}+1\right) / 2\right)\right.
$$

montre qu'il existe une représentation $\pi^{\prime}$ et une inclusion:

$$
\pi_{\epsilon} \hookrightarrow S D(\rho,(b-1) / 2,(a+1) / 2) \times \pi^{\prime} .
$$

Il est facile de vérifier que:

$$
\lambda_{\pi_{\epsilon}}(\rho, b)=\lambda_{\pi_{\epsilon}}\left(\rho, b^{\prime \prime}\right) .
$$

On conclut donc que si $\epsilon_{\pi_{0}}(\rho, b) \neq \epsilon_{\pi_{0}}(\rho, a)$ alors il en est de même pour les facteurs $\lambda_{\pi_{0}}$. L'égalité des facteurs $\lambda_{\pi_{0}}(\rho, b)=\lambda_{\pi_{0}}(\rho, a)$ quand on l'égalité des facteurs $\epsilon_{\pi_{0}}$ a déjà été vue puisque $a=b_{-}$.

Cela termine la preuve de (i). La preuve de (ii) est complètement immédiate, car pour tout $(\rho, a) \in \operatorname{Jord}\left(\pi_{0}^{\prime}\right)$ soit $\rho \neq \rho^{\prime}$ soit le segment $[(a-1) / 2,-(a-1) / 2]$ est inclus (si $a<a_{-}^{\prime}$ ) dans le segment $\left[\left(a^{\prime}-1\right) / 2,-\left(a_{-}^{\prime}-1\right) / 2\right]$ soit il contient ce dernier segment (si $a>a^{\prime}$ ). Donc dans tous les cas, l'induite:

$$
S D(\rho, a) \times S D\left(\rho^{\prime},\left(a^{\prime}-1\right) / 2,-\left(a_{-}^{\prime}-1\right) / 2\right)
$$

est irréductible. Et on vérifie sur les définitions l'égalité:

$$
\lambda_{\pi_{0}}(\rho, a)=\lambda_{\pi_{0}^{\prime}}(\rho, a) .
$$

D’où (ii) grâce à (i). 


\section{Exhaustivité première partie}

7.1 Rappelons qu'à une série discrète $\pi_{0}$, on a associé son support cuspidal partiel $\pi_{\text {cusp }}$, ses blocs de Jordan, $\operatorname{Jord}\left(\pi_{0}\right)$ et la fonction $\epsilon_{\pi_{0}}()$ bien définie pour tous les couples $(\rho, a) \in \operatorname{Jord}\left(\pi_{0}\right)$ tels que soit $a$ est pair soit pour tout $a^{\prime} \in \mathbf{N}$, $\left(\rho, a^{\prime}\right) \notin \operatorname{Jord}\left(\pi_{\text {cusp }}\right)$. Dans le cas restant on ne sait que définir les différences:

$$
\epsilon_{\pi_{0}}(\rho, a) \epsilon_{\pi_{0}}\left(\rho, a^{\prime}\right)^{-1} ;(\rho, a),\left(\rho, a^{\prime}\right) \in \operatorname{Jord}\left(\pi_{0}\right)
$$

Théorème: L'application qui à une série discrète $\pi_{0}$ associe $\pi_{c u s p}$, Jord $\left(\pi_{0}\right)$ et $\epsilon_{\pi_{0}}$ partiellement défini comme ci-dessus, est injective.

On le démontre évidemment par récurrence; on suppose d'abord que $\pi_{0}$ est fortement positive, alors la proposition 4.1 dit que $\pi_{0}$ est uniquement caractérisée par $\pi_{\text {cusp }}$ et $\operatorname{Jord}\left(\pi_{0}\right)$. On a d'ailleurs expliqué comment on calculait $\epsilon_{\pi_{0}}$ (toujours partiellement) grâce à ces données, mais cela n'est pas utile ici et ne servira que pour la surjectivité faite en [M-T].

Supposons donc que $\pi_{0}$ n'est pas fortement positive; il existe donc $(\rho, a) \in$ $\operatorname{Jord}\left(\pi_{0}\right)$ tel que $a_{-}$soit défini et

$$
\epsilon_{\pi_{0}}(\rho, a)=\epsilon_{\pi_{0}}\left(\rho, a_{-}\right)
$$

On sait alors qu'il existe une série discrète $\pi_{0}^{\prime}$ avec une inclusion:

$$
\pi_{0} \hookrightarrow S D\left(\rho,(a-1) / 2,-\left(a_{-}-1\right) / 2\right) \times \pi_{0}^{\prime} .
$$

Soit $\pi_{1}$ une série discrète de $G(n, F)$ ayant les mêmes données que $\pi_{0}$; i.e.: $\pi_{c u s p}$, $\operatorname{Jord}\left(\pi_{1}\right)=\operatorname{Jord}\left(\pi_{0}\right)$ et $\epsilon_{\pi_{1}}=\epsilon_{\pi_{0}}$. Et montrons que $\pi_{1} \simeq \pi_{0}$. Revenons à $\pi_{0}^{\prime}$ défini par (1). On sait que $\pi_{c u s p}$ est le support cuspidal partiel de $\pi_{0}^{\prime}$ mais on a aussi:

$$
\operatorname{Jord}\left(\pi_{0}^{\prime}\right)=\operatorname{Jord}\left(\pi_{0}\right)-\left\{(\rho, a),\left(\rho, a_{-}\right)\right\}
$$

et $\epsilon_{\pi_{0}^{\prime}}$ est la restriction à $\operatorname{Jord}\left(\pi_{0}^{\prime}\right)$ de $\epsilon_{\pi_{0}}$. Ainsi, avec l'hypothèse de récurrence, on a aussi:

$$
\pi_{1} \hookrightarrow S D\left(\rho,(a-1) / 2,-\left(a_{-}-1\right) / 2\right) \times \pi_{0}^{\prime} .
$$

Or on sait que (1) a exactement 2 sous-modules irréductibles et nous n'avons de problème que si ces 2 sous-modules sont des séries discrètes; toutefois pour ce qui suit nous notons $\pi_{1}$ l'autre sous-module et nous allons le distinguer de $\pi_{0}$ par une propriété de ses modules de Jacquet qui ne suppose pas que l'on sache que $\pi_{1}$ est une série discrète.

Supposons d'abord qu'il existe $b \in \mathbf{N}$ vérifiant $(\rho, b) \in \operatorname{Jord}\left(\pi_{0}^{\prime}\right)$. On fixe un tel $b$. S'il est supérieur à $a$, on lui impose d'être minimal avec ces propriétés; s'il est inférieur à $a_{-}$, on lui impose d'être maximal avec ces propriétés. Quand les 2 choix sont possibles, on fait n'importe lequel. Et on va montrer que les 2 sous-modules irréductibles de (1) sont séparés par

$$
\epsilon_{?}(\rho, a) \epsilon_{?}(\rho, b)^{-1}
$$


Si on ne sait pas que $\pi_{1}$ est une série discrète, cela veut dire que l'on va montrer que soit $\pi_{0}$ soit $\pi_{1}$ et seulement l'une des 2 représentations est sous-module d'une induite:

$$
S D(\rho,(b-1) / 2,-(a-1) / 2) \times \pi^{\prime},
$$

où $\pi^{\prime}$ est une série discrète convenable dans le cas où $b>a$ et dans l'autre cas on remplace $S D(\rho,(b-1) / 2,-(a-1) / 2) \operatorname{par} S D\left(\rho,\left(a_{-}-1\right) / 2,-(b-1) / 2\right)$.

On regarde d'abord le cas où $b<a_{-}$; on montre par un calcul de module de Jacquet l'équivalence:

$$
\epsilon_{\pi_{0}}\left(\rho, a_{-}\right)=\epsilon_{\pi_{0}}(\rho, b) \Leftrightarrow
$$

$$
\pi_{0} \hookrightarrow S D(\rho,(a-1) / 2,-(b-1) / 2) \times S D\left(\rho,\left(a_{-}-1\right) / 2,(b+1) / 2\right) \times \pi_{0}^{\prime} .
$$

Or le terme de droite de (3) a un unique sous-module irréductible (l'unicité vient du fait que $\left.(\rho, b) \in \operatorname{Jord}\left(\pi_{0}^{\prime}\right)\right)$. Et en regardant les modules de Jacquet de:

$$
S D\left(\rho, a_{-}\right) \times \pi_{0}^{\prime}
$$

on vérifie que ce sous-module est aussi sous-module de

$$
S D\left(\rho,(a-1) / 2,\left(a_{-}+1\right) / 2\right) \times S D\left(\rho, a_{-}\right) \times \pi_{0}^{\prime}
$$

puis de (1). Ainsi si $\pi_{0}$ vérifie l'égalité en (2), il est uniquement caractérisé tandis que s'il ne la vérifie pas et s'il n'est pas égal à $\pi_{1}$ c'est $\pi_{1}$ qui vérifie cette égalité (ou plutôt son analogue en terme de module de Jacquet). Cela contredirait l'égalité des fonctions $\epsilon_{\pi_{0}}$ et $\epsilon_{\pi_{1}}$.

Supposons maintenant que $b>a$. On a déjà vu (cf. 5.4.2) qu'il existe une série discrète $\pi_{1}^{\prime}$ et une inclusion:

$$
\pi_{0}^{\prime} \hookrightarrow S D\left(\rho,(b-1) / 2,\left(a_{-}+1\right) 2\right) \times \pi_{1}^{\prime} .
$$

Et on note $<S D\left(\rho, a_{-}\right) \times S D\left(\rho,(b-1) / 2,\left(a_{-}+1\right) / 2>1\right.$ 'unique sous-module de l'induite écrite entre les crochets. On considère les 2 constituants de:

$$
S D\left(\rho, a_{-}\right) \times \pi_{0}^{\prime}
$$

que l'on note $\tau_{\epsilon}$ pour $\epsilon= \pm 1$. Fixons $\epsilon$, alors soit

$$
\tau_{\epsilon} \hookrightarrow<S D\left(\rho, a_{-}\right) \times S D\left(\rho,(b-1) / 2,\left(a_{-}+1\right) / 2>\times \pi_{1}^{\prime},\right.
$$

soit

$$
\tau_{\epsilon} \hookrightarrow S D\left(\rho,(b-1) / 2,-\left(a_{-}-1\right) / 2\right) \times \pi_{1}^{\prime} .
$$

En regardant les modules de Jacquet, on vérifie que l'induite totale $S D\left(\rho, a_{-}\right) \times \pi_{0}^{\prime}$ n'est inclus ni dans (4) ni dans (5). Cela veut dire que pour une valeur de $\epsilon$ (4) est satisfait et pour l'autre c'est (5) qui est satisfait. Les 2 sous-modules de l'induite (1) sont alors distingués parce que l'un est inclus dans:

$S D\left(\rho,(a-1) / 2,\left(a_{-}+1\right) / 2 \times<S D\left(\rho, a_{-}\right) \times S D\left(\rho,(b-1) / 2,\left(a_{-}+1\right) / 2>\times \pi_{1}^{\prime}\right.\right.$ 
alors que l'autre est inclus dans

$$
S D\left(\rho,(a-1) / 2,\left(a_{-}+1\right) / 2 \times S D\left(\rho,(b-1) / 2,-\left(a_{-}-1\right) / 2\right) \times \pi_{1}^{\prime} .\right.
$$

Dans le module de Jacquet de l'induite écrite en (6), on ne trouve pas de terme du type:

$$
\left.\left.\rho\right|^{(b-1) / 2} \otimes \cdots \otimes \rho||^{(b-1) / 2-i+1} \otimes \cdots \otimes \rho\right|^{(a+1) / 2} \otimes \cdots,
$$

alors que tout sous-module de (7) en contient 1 à cause de l'irréductibilité de l'induite:

$$
S D\left(\rho,(a-1) / 2,\left(a_{-}+1\right) / 2 \times S D\left(\rho,(b-1) / 2,-\left(a_{-}-1\right) / 2\right) .\right.
$$

Ainsi les sous-modules de (1) sont distingués par

$$
\epsilon_{?}(\rho, b) \epsilon_{?}(\rho, a)^{-1} \text {. }
$$

Cela termine la preuve dans ce cas.

Il reste le cas le plus simple, qui est un cas particulier du cas où $\epsilon_{\pi_{0}}(\rho$, ?) est complètement défini. Supposons cela. Alors les 2 sous-modules de (1) sont séparés par la valeur de l'opérateur d'entrelacement en $s=0$ agissant sur la grosse induite:

$$
\begin{aligned}
& S D\left(\rho,(a-1) / 2,\left(a_{-}+1\right) / 2\right) \times S D\left(\rho, a_{-}\right)||^{s} \times \pi_{0}^{\prime} \rightarrow \\
& S D\left(\rho,(a-1) / 2,\left(a_{-}+1\right) / 2\right) \times\left. S D\left(\rho, a_{-}\right)\right|^{-s} \times \pi_{0}^{\prime} .
\end{aligned}
$$

On est dans le cas où pour tout $a^{\prime} \in \mathbf{N},\left(\rho, a^{\prime}\right) \notin \operatorname{Jord}\left(\pi_{\text {cusp }}\right)$. Sous cette hypothèse, on a précisément défini $\epsilon_{\pi_{0}}(\rho, a)$ à l'aide de cette action; cela prouve que puique $\epsilon_{\pi_{0}}(\rho, a)=\epsilon_{\pi_{1}}(\rho, a), \pi_{1}$ et $\pi_{0}$ coïncident.

\subsection{Exhaustivité 2e version}

Le théorème précédent suffit pour classifier les séries discrètes; mais on a quand même envie de n'utiliser que des données du $L$-groupe pour classifier ces séries discrètes; dans le théorème précédent, il reste $\pi_{c u s p}$, le support cuspidal partiel qui n'est pas traduit en terme de $L$-groupe. Pour pouvoir faire quelque chose dans le cas des séries discrètes, il faut avant tout traiter le cas cuspidal. Avec notre traduction combinatoire, pour tout $\pi$ cuspidal, on veut savoir construite $\operatorname{Jord}(\pi)$ et $\epsilon_{\pi}(\rho, a)$ pour tout $(\rho, a) \in \operatorname{Jord}(\pi)$. Et on voudrait que l'application qui à $\pi$ associe ces données soit injective.

Rappelons, que l'on a défini $\operatorname{Jord}(\pi)$ (pour avoir de bonnes propriétés, on a utilisé notre hypothèse sur les points de réductibilité des induites de cuspidales). Et on a défini une bonne partie de $\epsilon_{\pi}$. Il reste à définir $\epsilon_{\pi}(\rho, 1)$ quand $(\rho, 1) \in \operatorname{Jord}(\pi)$. Dans les idées de Langlands, la restriction du caractère du centralisateur au centre du $L$-groupe est imposée (prendre 1 n'est pas forcément une bonne idée mais elle est certainement à imposer ne dépendant que de $G(n)$ ). Cela veut dire que l'on a plus guère de choix. Dans le cas des représentations quadratiques unipotentes cuspidales de [M2], j'ai bien montré comment on pouvait définir l'application $\epsilon_{\pi}$ en ayant la propriété d'injectivité (et même de bijectivité) cherchée; j'avais utilisé le 
front d'onde des représentations que je savais (essentiellement) calculer grâce à la correspondance de Howe. Il est très probable que le cas quadratique unipotent soit tout à fait analogue au cas général, mais on ne dispose pas de moyen pour faire des récurrences. La direction la plus prometteuse semble bien être la normalisation des opérateurs d'entrelacement comme suggéré dans la remarque 6.1.3. Cela justifie l'hypothèse suivante:

pour tout $\pi$ cuspidale on peut définir $\epsilon_{\pi}$ comme fonction sur $\operatorname{Jord}(\pi)$ à valeurs dans $\{ \pm 1\}$ satisfaisant les conditions déjà imposées et telle que l'application:

$$
\pi \mapsto \operatorname{Jord}(\pi), \epsilon_{\pi}
$$

soit injective sur l'ensemble des représentations cuspidales de $G(n, F)$.

On admet donc cette hypothèse, pour tout $n^{\prime} \leq n$, dans ce paragraphe et on complète la définition de $\epsilon_{\pi_{0}}$ pour toute série discrète de $G(n, F)$.

Pour cela on remarque d'abord qu'il y a au plus une définition de $\epsilon_{\pi_{0}}$ qui satisfait aux 2 conditions suivantes:

si $\pi_{0}$ est fortement positive et si $(\rho, a) \in \operatorname{Jord}\left(\pi_{0}\right)$ tel qu'il existe une série discrète $\pi_{0}^{\prime}$ et une inclusion:

$$
\pi_{0} \hookrightarrow \rho||^{(a-1) / 2} \times \pi_{0}^{\prime}
$$

alors pour tout $\left(\rho^{\prime}, a^{\prime}\right) \in \operatorname{Jord}\left(\pi_{0}\right)$ avec $\left(\rho, a^{\prime}\right) \neq(\rho, a)$ :

$$
\epsilon_{\pi_{0}}\left(\rho^{\prime}, a^{\prime}\right)=\epsilon_{\pi_{0}^{\prime}}\left(\rho^{\prime}, a^{\prime}\right)
$$

et $\epsilon_{\pi_{0}}(\rho, a)=\epsilon_{\pi_{0}^{\prime}}(\rho, a-2)$.

Si $\pi_{0}$ n'est pas fortement positive et si $(\rho, a) \in \operatorname{Jord}\left(\pi_{0}\right)$ avec $a_{-}$défini et l'existence d'une série discrète $\pi_{0}^{\prime}$ avec une inclusion:

$$
\pi_{0} \hookrightarrow S D\left(\rho,(a-1) / 2,-\left(a_{-}-1\right) / 2\right) \times \pi_{0}^{\prime}
$$

alors $\epsilon_{\pi_{0}^{\prime}}=\left(\epsilon_{\pi}\right)_{\mid \operatorname{Jord}\left(\pi_{0}^{\prime}\right)}$.

La première condition impose uniquement la façon de définir $\epsilon_{\pi_{0}}$ si $\pi_{0}$ est fortement positive. Et la proposition 4.1, montre que l'on peut effectivement définir $\epsilon_{\pi_{0}}$ dans ce cas.

Supposons maintenant que $\pi_{0}$ ne soit pas fortement positive. On sait que l'on peut trouver $(\rho, a) \in \operatorname{Jord}\left(\pi_{0}\right)$ tel que $a_{-}$soit défini et tel qu'il existe une série discrète $\pi_{0}^{\prime}$ avec une inclusion:

$$
\pi_{0} \hookrightarrow S D\left(\rho,(a-1) / 2,-\left(a_{-}-1\right) / 2\right) \times \pi_{0}^{\prime} .
$$

On définit $\epsilon_{\pi_{0}}$ à l'aide de $\epsilon_{\pi_{0}^{\prime}}$ de façon à ce que la deuxième propriété soit satisfaite pour cette inclusion. Il y a alors à démontrer que cette définition est indépendante du choix fait de $(\rho, a)$. Je le laisse au lecteur, cela se fait par les méthodes élémentaires utilisées dans cet article.

On peut maintenant supprimer $\pi_{c u s p}$ de la première version du théorème d'exhaustivité:

Théorème: Avec les hypothèses faites dans le cas cuspidal, l'application $\pi_{0} \mapsto$ $\operatorname{Jord}\left(\pi_{0}\right), \epsilon_{\pi_{0}}$ est injective sur l'ensemble des séries discrètes de $G(n, F)$.

C'est exactement la démonstration de 7.1. 


\section{Appendice}

Dans cet appendice, on traite d'abord le cas des groupes unitaires puis celui des groupes orthogonaux "pairs". Ici donc $G$ sera un groupe $U(n, E / F)$ où $n \in \mathbf{N}$ et $E$ est une extension quadratique de $F$; la notation ne fait pas intervenir la forme unitaire, bien que le groupe en dépende. Notre définition des blocs de Jordan n'est qu'une traduction combinatoire commode des paramètres de Langlands. Elle a l'avantage d'éviter toute conjecture locale sur la paramétrisation des cuspidales. Toutefois, la paramétrisation de Langlands nous sert de guide et dans un premier temps, on va classifier l'ensemble des homomorphismes:

$$
\phi: W_{F} \times S L(2, \mathbf{C}) \rightarrow{ }^{L} G
$$

considéré à conjugaison près et ayant la propriété que

$$
\mid \text { Cent }_{L} G^{0} \phi \mid<\infty
$$

et tel que

$\phi$ composé avec la projection sur $\operatorname{Gal}(E / F)$ coïncide avec la projection de $W_{F}$ sur ce groupe;

la restriction de $\phi$ à $W_{F}$ est continue et formée d'éléments semi-simples tandis que la restriction de $\phi$ à $S L(2, \mathbf{C})$ est algébrique.

Pour classifier ces morphismes, on définit $\operatorname{Jord}(\phi)$ comme l'ensemble des couples $(\rho, a)$ formé d'une représentation irréductible de $W_{E}$ et d'un entier $a$ précisément tels que, en notant $E_{a}$ la représentation irréductible de $\operatorname{SL}(2, \mathbf{C})$ de dimension $a, \rho \otimes E_{a}$ intervient dans la restriction de $\phi$ à $W_{E} \times S L(2, \mathbf{C})$. On montre que si $(\rho, a) \in \operatorname{Jord}(\phi)$, il intervient avec multiplicité 1 et vérifie les propriétés:

on fixe $\theta \in W_{E}-W_{F}$ et on demande l'isomorphisme des représentations:

$$
x \in W_{E} \mapsto \rho\left(\theta x \theta^{-1}\right) \simeq \rho^{*}
$$

Un tel isomorphisme permet d'associer un signe à la représentation $\rho$, noté $\lambda_{\rho}$. Il est tel que pour $A_{\rho} \in G L\left(d_{\rho}, \mathbf{C}\right)$ vérifiant pour tout $x \in W_{E}$ :

$$
\begin{gathered}
A_{\rho}{ }^{t} \rho\left(x^{-1}\right) A_{\rho}^{-1}=\rho(x) \\
A_{\rho}{ }^{t} A_{\rho}^{-1}=\lambda_{\rho} I d .
\end{gathered}
$$

la dernière condition est que $a$ est paire si $\lambda_{\rho}=(-1)^{n}$ et impaire sinon.

Les homomorphismes $\rho$ ne sont considérés qu'à conjugaison près dans $G L\left(d_{\rho}, \mathbf{C}\right)$.

Et on a la condition de dimension: $\sum_{(\rho, a) \in \operatorname{Jord}(\phi)} a d_{\rho}=n$.

On montre que l'application $\phi \mapsto \operatorname{Jord}(\phi)$ est injective (modulo conjugaison) d'image la totalité des ensembles de couples ayant les propriétés ci-dessus.

En outre, nous allons montrer que pour $\phi$ comme ci-dessus auquel nous venons d'associer l'ensemble $\operatorname{Jord}(\phi)$, il y a un isomorphisme entre l'ensemble des caractères du centralisateur de $\phi$ dans ${ }^{L} G^{0}$ et les applications de $\operatorname{Jord}(\phi)$ dans $\{ \pm 1\}$. 
Considérons maintenant une série discrète irréductible, $\pi_{0}$ de $G$. On définit a priori $\operatorname{Jord}\left(\pi_{0}\right)$ de la façon suivante: $\operatorname{Jord}\left(\pi_{0}\right)$ est un ensemble de couples (sans multiplicité) $(\rho, a)$ où $\rho$ est une représentation cuspidale irréductible de $G L\left(d_{\rho}, E\right)$ (cela définit $d_{\rho}$ ) et $a \in \mathbf{N}$ vérifiant les hypothèses:

on note $\sigma$ l'élément non trivial de $\operatorname{Gal}(E / F)$ et on demande que les représentations $\rho$ qui apparaissent vérifient:

$$
{ }^{\sigma} \rho \simeq \rho^{*} .
$$

Il y a aussi une condition sur la parité de $a$ que l'on expliquera ci-dessous et on demande que l'induite pour le groupe $U(n+2 a, E / F)$ :

$$
S D(\rho, a) \times \pi_{0}
$$

soit irréductible, où $S D(\rho, a)$ est la série discrète de $G L\left(d_{\rho} a, E\right)$ basée sur $\rho$.

La condition de parité dépend de la représentation $\rho$; on considère le $L$-groupe de $G L\left(d_{\rho}, E\right)$ vu comme un $F$-groupe, c'est-à-dire

$$
\left(G L\left(d_{\rho}, \mathbf{C}\right) \times G L\left(d_{\rho}, \mathbf{C}\right)\right) \ltimes G a l(E / F)
$$

et pour $\eta= \pm 1$ on définit la représentaion $R_{\eta}$ de ce groupe sur End $\mathbf{C}^{d_{\rho}}$ par:

$$
\forall g, g^{\prime} \in G L\left(d_{\rho}, \mathbf{C}\right), x \in E n d \mathbf{C}^{d_{\rho}}, \quad\left(g, g^{\prime}, 1\right) . x=g x^{t} g^{\prime} \quad(1,1, \sigma) . x={ }^{t} x .
$$

La condition de parité sur $a$ pour que $(\rho, a)$ puisse intervenir dans $\operatorname{Jord}\left(\pi_{0}\right)$ est que $a$ doit être pair si $L\left(\rho, R_{(-1)^{n}}, s\right)$ a un pôle en $s=0$ et impair sinon.

Cette représentation $R_{(-1)^{n}}$ ne sort pas de mon chapeau mais de la représentation adjointe du $L$-groupe de $U\left(n+2 d_{\rho}, E / F\right)$.

\section{A.1 Classification des homomorphismes de $W_{F} \times S L(2, \mathbf{C})$ dans ${ }^{L} G$}

Rappelons la description standard $\operatorname{de}^{L} G$ : on l'identifie au produit semi-direct:

$$
{ }^{L} G \simeq G L(n, \mathbf{C}) \ltimes G a l(E / F),
$$

où l'action de l'élément non trivial $\sigma$ de $\operatorname{Gal}(E / F)$ se fait sur $G L(n, \mathbf{C})$ par:

$$
\forall g \in G L(n, \mathbf{C}), \quad \sigma(g):=\alpha^{t} g^{-1} \alpha^{-1},
$$

où $\alpha$ est la matrice:

$\alpha:=\begin{array}{ccccccc}0 & 0 & 0 & \cdots & 0 & 0 & 1 \\ 0 & 0 & 0 & \cdots & 0 & -1 & 0 \\ 0 & 0 & 0 & \cdots & 1 & 0 & 0 \\ . & . & . & & . & . & . \\ . & . & . & & . & . & . \\ . & . & . & & . & . & . \\ 0 & 0 & (-1)^{n-3} & \cdots & 0 & 0 & 0 \\ 0 & (-1)^{n-2} & 0 & \cdots & 0 & 0 & 0 \\ (-1)^{n-1} & 0 & 0 & \cdots & 0 & 0 & 0\end{array}$


Soit $\phi: W_{F} \rightarrow^{L} G$ dont l'image est formé d'élément semi-simple et tel que le diagramme:

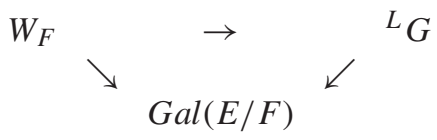

La restriction de $W_{F}$ à $W_{E}$ est un homomorphisme continu de $W_{E}$ dans $G L(n, \mathbf{C})$. D'où une représentation $\phi_{E}$ dans $V:=\mathbf{C}^{d_{\rho}}$ que l'on décompose en composantes isotypiques:

$$
V=: \oplus_{\{\rho\}} V[\rho],
$$

où $\rho$ parcourt un ensemble de représentation irréductible de $W_{E}$. On fixe $\theta \in$ $W_{F}-W_{E}$. Ecrivons:

$$
\phi(\theta)=:\left(A \alpha^{-1}, \sigma\right),
$$

où $\sigma$ est l'élément non trivial de $\operatorname{Gal}(E / F)$ et où $A \in G L(n, \mathbf{C})$. Le fait que $\phi$ soit un homomorphisme se traduit par les 2 relations suivantes:

$$
\begin{gathered}
\forall x \in W_{E}, \quad \phi\left(\theta x \theta^{-1}\right)=A^{t} \phi\left(x^{-1}\right) A^{-1} ; \\
\phi\left(\theta^{2}\right)=A^{t} A^{-1} \alpha^{-1} \alpha .
\end{gathered}
$$

On remarque que ${ }^{t} \alpha^{-1}=\alpha$ et que $\alpha^{2}$ est la matricie scalaire $(-1)^{n-1}$. La deuxième condition se récrit donc:

$$
\phi\left(\theta^{2}\right)=(-1)^{n-1} A^{t} A^{-1} .
$$

Ecrivons l'action par conjugaison de ${ }^{L} G$ : l'élément $\sigma^{\prime}:=\left(\alpha^{-1}, \sigma\right)$ agit par:

$$
\begin{gathered}
\forall x \in W_{E}, \quad \sigma^{\prime} \phi(x) \sigma^{\prime-1}={ }^{t} \phi\left(x^{-1}\right) \\
\sigma^{\prime} \phi(\theta) \sigma^{\prime-1}=\left({ }^{t} A^{-1} \alpha^{-1}, \sigma\right) .
\end{gathered}
$$

En d'autres termes $\phi_{E}$ est transformé en ${ }^{t} \phi_{E}^{-1}$ et $A$ en ${ }^{t} A^{-1}$. L'action des éléments du type $(B, 1)$ est simplement la conjugaison dans $G L(n, \mathbf{C})$ en ce qui concerne $\phi_{E}$. Et:

$$
(B, 1) \phi(\theta)\left(B^{-1}, 1\right)=\left(B A^{t} B \alpha^{-1}, \sigma\right) .
$$

En d'autres termes $A$ est envoyé sur $B A^{t} B$.

La condition (1) entraîne que les représentations $\phi_{E}$ et ${ }^{\theta} \phi_{E}^{-1}$ sont isomorphes. Donc si $\rho$ intervient dans la décomposition de $\phi_{E}$, i.e. $V[\rho] \neq 0$, alors $V\left[{ }^{\theta} \rho^{*}\right] \neq 0$. Deux cas sont possibles:

le premier cas est celui où ${ }^{\theta} \rho \not \rho^{*}$. On pose alors $\rho^{\prime}:=\theta^{-1} \rho^{*}$ et pour $z \in \mathbf{C}$, on considère l'élément de $G L(n, \mathbf{C})$ qui agit par le scalaire 1 pour tout $\rho^{\prime \prime}$ non isomorphe à $\rho$ ou $\rho^{\prime}$, par le scalaire $z$ sur $V[\rho]$ et le scalaire $z^{-1}$ sur $V\left[\rho^{\prime}\right]$. Cet élément est clairement dans le centre du centralisateur de $\phi$ dans ${ }^{L} G^{0}$. Ainsi ce centralisateur n'est pas à centre fini. Et quelque soit le prolongement de $\phi$ en un homomorphisme de $W_{F} \times S L(2, \mathbf{C})$ dans ${ }^{L} G$, le centralisateur contiendra ce groupe d'éléments et ne sera pas fini.

Le seul cas qui nous intéresse est donc celui où ${ }^{\theta} \rho \simeq \rho^{*}$. 
On suppose donc que pour tout $\rho$ tel que $V[\rho] \neq 0, \rho \simeq^{\theta} \rho^{*}$. On fixe une réalisation $V_{\rho}$ de $\rho$ et on réalise $\rho^{*}$ dans $V_{\rho}$ en utilisant la formule habituelle (dûe à Kazdhan, sauf erreur), ${ }^{t} \rho^{-1}$. Evidemment la transposition est celle qui vient du choix d'une base de $V_{\rho}$. On écrit explicitement:

$$
V[\rho]=V_{\rho} \oplus \cdots \oplus V_{\rho},
$$

avec un choix de base naturellement imposé par celui fait sur $V_{\rho}$. En outre, pour tout $\rho$, on fixe $A_{\rho} \in \operatorname{Aut}\left(V_{\rho}\right)$ entrelaçant les représentations ${ }^{\theta} \rho$ et ${ }^{t} \rho^{-1}$, précisément, pour tout $x \in W_{E}$ :

$$
\rho\left(\theta x \theta^{-1}\right)=A_{\rho}^{t} \rho\left(x^{-1}\right) A_{\rho}^{-1} .
$$

On remarque que $A_{\rho}$ est uniquement déterminé à un scalaire près. En revenant à (3) et en y remplaçant $x$ par $\theta y \theta^{-1}$, on voit que pour tout $y \in W_{E}$ :

$$
\begin{gathered}
\rho\left(\theta^{2}\right) \rho(y) \rho\left(\theta^{2}\right)^{-1}=A_{\rho}{ }^{t}\left(\rho\left(\theta y \theta^{-1}\right)\right)^{-1} A_{\rho}^{-1}= \\
A_{\rho}{ }^{t} A_{\rho}^{-1} \rho(y)^{t} A_{\rho} A_{\rho}^{-1} .
\end{gathered}
$$

Ce qui entraîne que $\rho(\theta)^{-2} A_{\rho}{ }^{t} A_{\rho}^{-1}$ est dans le commutant de la représentation de $\rho$; cet opérateur est donc scalaire. Notons $\lambda_{\rho}$ ce scalaire. D'où encore:

$$
A_{\rho}=\lambda_{\rho} \rho\left(\theta^{2}\right)^{t} A_{\rho}
$$

En transposant:

$$
{ }^{t} A_{\rho}=\lambda_{\rho} A_{\rho}{ }^{t} \rho\left(\theta^{2}\right) .
$$

D'où:

$$
A_{\rho}=\lambda_{\rho}^{2} \rho\left(\theta^{2}\right) A_{\rho}{ }^{t} \rho\left(\theta^{2}\right)=\lambda_{\rho}^{2} \rho\left(\theta^{2}\right) \rho\left(\theta^{-2}\right) A_{\rho} .
$$

C'est-à-dire: $\lambda_{\rho}^{2}=1$.

On définit $A_{0} \in A u t V$ de tel sorte que $A_{0}$ respecte chaque espace $V[\rho]$ et que sur chaque $V[\rho]$, il induise la sommes des opérateurs $A_{\rho}$ plongé diagonalement en fonction la décomposition choisie. D'où:

$$
A_{0}{ }^{t} \phi\left(x^{-1}\right) A_{0}^{-1}=\phi\left(\theta x \theta^{-1}\right) .
$$

Revenons à $\phi(\theta)$ ce qui est équivalent à la donnée de l'opérateur $A$ satisfaisant les conditions 1 et 2 ci-dessus. Et on pose

$$
A_{\theta}:=A A_{0}^{-1} .
$$

Les relations deviennent:

$$
\forall x \in W_{E}, \quad A_{\theta} A_{0}{ }^{t} \phi\left(x^{-1}\right) A_{0}^{-1} A_{\theta}^{-1}=\phi\left(\theta x \theta^{-1}\right) .
$$

Par (4), pour tout $x \in W_{E}$,

$$
A_{\theta} \phi\left(\theta x \theta^{-1}\right) A_{\theta}^{-1}=\phi\left(\theta x \theta^{-1}\right) .
$$


Ainsi la relation 1 est équivalente à ce que $A_{\theta}$ soit dans le commutant de la représentation $\phi_{E}$ de $W_{E}$.

Quant à la relation 2, elle s'écrit:

$$
\phi\left(\theta^{2}\right)=(-1)^{n-1} A_{\theta} A_{0}{ }^{t} A_{0}^{-1 t} A_{\theta}^{-1} .
$$

Sur chaque $V[\rho], A_{0}{ }^{t} A_{0}^{-1}$ est le produit de la restriction de $\phi\left(\theta^{2}\right)$ par le scalaire $\lambda_{\rho}$. En outre, la relation 1 indique que $A_{\theta}$ commute à $\phi\left(\theta^{2}\right)$. Ainsi les relations 1 et 2 sont équivalente d'une part à la relation de commutation pour $A_{\theta}$ et d'autre part à:

$$
\forall \rho \mid V[\rho] \neq 0, \quad A_{\theta}=(-1)^{n-1} \lambda_{\rho}{ }^{t} A_{\theta} .
$$

A conjugaison près (attention c'est la conjugaison par $B A_{\theta}{ }^{t} B$ ), il y a au plus une matrice $A_{\theta}$ possible. Elle est symétrique si $(-1)^{n-1}=\lambda_{\rho}$ et antisymétrique sinon, ce qui force alors la multiplicité de $\rho$ à être pair. Ainsi les homomorphismes (tels que nous les avons considérés) de $W_{F}$ dans ${ }^{L} G$, considérés à conjugaison près, sont classifiés par la donnée des représentations irréductibles $\rho$ de $W_{E}$ intervenant dans la restriction de $\phi$ à $W_{E}$ avec leur multiplicité. Les représentations $\rho$ doivent être isomorphes à ${ }^{\theta} \rho^{*}$ et la multiplicité doit être paire si $(-1)^{n-1} \neq \lambda \rho$.

Cela termine la classification mais nous n'avons pas distingué les homomorphismes dont le centralisateur est fini; cela va être fait ci-dessous.

Calcul du commutant de $\phi\left(W_{F}\right)$ :

Soit $g \in \operatorname{Aut}(V)$ une matrice commutant à $\phi\left(W_{F}\right)$. Comme $g$ commute à $\phi\left(W_{E}\right)$, elle respecte la décomposition en espace isotypique. Pour $\rho$ fixé, notons $g_{\rho}$ la restriction de $g$ à $V[\rho]$. Et on a:

$$
g_{\rho}=\phi(\theta) g_{\rho} \phi\left(\theta^{-1}\right)=A_{\theta} A_{0}{ }^{t} g_{\rho}^{-1} A_{0}^{-1} A_{\theta}^{-1} .
$$

Comme $g_{\rho}$ commute $\phi\left(W_{E}\right)$, cet opérateur commute aux élément de $G L\left(V_{\rho}\right)$ plongé diagonalement dans $\operatorname{Aut}(V[\rho])$. Donc $g_{\rho}$ commute à $A_{0}$ et on obtient:

$$
g_{\rho} A_{\theta}{ }^{t} g_{\rho}=A_{\theta} .
$$

En d'autres termes, le commutant de $\phi\left(W_{F}\right)$ est le produit

$$
\times_{\rho \in W_{E} ; V[\rho] \neq 0} C\left(\rho,\left[V[\rho]: V_{\rho}\right]\right)
$$

où $C(\rho)$ est un groupe orthogonal si $\lambda_{\rho}=(-1)^{n-1}$ et est un groupe symplectique sinon.

Supposons maintenant que $\phi$ se prolonge en un homomorphisme de

$$
W_{F} \times S L(2, \mathbf{C}) \rightarrow^{L} G .
$$

Avec les notations ci-dessus, l'image de $\phi(S L(2, \mathbf{C})$ se factorise par le groupe $\times{ }_{\rho} C(\rho)$. On rappelle que les représentations irréductibles de $S L(2, \mathbf{C})$ sont classifiées par leur dimension, et qu'une représentation de dimension paire est symplectique alors qu'une représentation de dimension impaire est orthogonale; pour 
$a \in \mathbf{N}$, on note $E_{a}$ la représentation irréductible de $S L(2, \mathbf{C})$ de dimension $a$. Notons momentanément $\phi_{\rho}$ le composé de $\phi_{\mid S L(2, \mathbf{C})}$ avec la projection sur $C(\rho)$; cette mauvaise notation ne restera pas. Alors $\phi_{\rho}$ se décompose en somme de représentations irréductibles éventuellement avec multiplicité. Pour $a \in \mathbf{N}$ fixé, écrivons $V[\rho, a]$ le sous-espace de $V[\rho]$ sur lequel la représentation de $S L(2, \mathbf{C})$ est isotypique de type $E_{a}$. Nécessairement $V[\rho, a]$ est symplectique si $\lambda_{\rho}=(-1)^{n}$ et orthogonal si $\lambda_{\rho}=(-1)^{n-1}$. En d'autre terme la multiplicité de $\rho \otimes E_{a}$ dans $V[\rho, a]$ est nécessairement paire si $a$ est impair (respectivement pair) alors que $\lambda_{\rho}=(-1)^{n}$ (resp. $\lambda_{\rho}=(-1)^{n-1}$.

On note $C(\rho, a)$ l'intersection de $C(\rho)$ avec le centralisateur de la représentation de $W_{E} \times S L(2, \mathbf{C})$ dans $V[\rho, a]$. Il est alors clair que:

$$
\operatorname{Cent}_{L} G^{0} \phi=\times_{\rho, a} C(\rho, a) .
$$

En outre $C(\rho, a)$ est fini si et seulement si $V[\rho, a]$ est irréductible comme représentation de $W_{E} \times S L(2, \mathbf{C})$; dans ce cas, $C(\rho, a)$ est le groupe à 2 éléments (centre du groupe symplectique $\operatorname{Sp}(a, \mathbf{C})$ ou du groupe orthogonal $O(a, \mathbf{C})$ suivant la parité de $a$ )

le cas où ${ }^{\theta} \rho \not \rho^{*}$.

Ici, on pose encore $\phi(\theta)=\left(A \alpha^{-1}, \sigma\right)$. Ainsi, pour tout $\rho \in \widehat{W_{E}}$ tel que $V[\rho] \neq 0, A$ induit un isomorphisme, $A_{\rho}$ de $V[\rho]$ sur $V\left[{ }^{\theta^{-1}} \rho^{*}\right]$. Soit $g$ un élément du commutant de $\phi\left(W_{E}\right)$ dans $A u t\left(V[\rho] \oplus V\left[{ }^{\theta^{-1}} \rho^{*}\right]\right.$. Il est clair que $g$ laisse stable $V[\rho]$ et $V\left[{ }^{\theta^{-1}} \rho^{*}\right]$; on note $g_{\rho}$ et $g_{\rho}^{*}$ ces 2 restrictions. Et on calcule:

$$
\phi(\theta)\left(g_{\rho} \oplus g_{\rho}^{*}\right) \phi(\theta)^{-1}=\left(A^{t} g^{*-1} A^{-1} \oplus A^{t} g^{-1} A^{-1}\right) .
$$

Cet élément est donc dans le commutant de $\phi\left(W_{F}\right)$ si et seulement si:

$$
g^{*}=A^{t} g^{-1} A^{-1} \text {. }
$$

Ce commutant est donc isomorphe à $G L\left(\left[V[\rho]: V_{\rho}\right], \mathbf{C}\right)$ où $\left[V[\rho]: V_{\rho}\right]$ est la multiplicité de $V_{\rho}$ dans $V[\rho]$.

Supposons encore que $\phi$ se prolonge en un morphisme de $W_{F} \times S L(2, \mathbf{C})$ dans ${ }^{L} G$; évidemment l'image de $S L(2, \mathbf{C})$ est dans le commutant de $\phi\left(W_{F}\right)$ et laisse donc stable $V[\rho]$ et $V\left[{ }^{\theta^{-1}} \rho^{*}\right]$. Sur la somme de ces espaces, $\phi(S L(2, \mathbf{C})$ s'identifie à un sous-groupe de $G L\left(\left[V[\rho]: V_{\rho}\right], \mathbf{C}\right)$, donc à une représentation semi-simple de dimension $\left[V[\rho]: V_{\rho}\right]$. Il n'y a aucune autre condition mais le centralisateur n'est pas fini car il contient au moins le centre de ce groupe linéaire.

Cela termine la classification des homomorphismes de $W_{F} \times S L(2, \mathbf{C})$ dans ${ }^{L} G$ considérés et le calcul de leur centralisateurs. 


\section{A.2 Le côté des représentations pour les groupes unitaires}

A.2.1 Les définitions. Etant donné ce que l'on vient de voir du côté L-groupe, il est naturel de vouloir classifier les séries discrètes, $\pi_{0}$, des groupes unitaires, $U(n, E / F)$ à l' aide d'un ensemble de blocs de Jordan, $\operatorname{Jord}\left(\pi_{0}\right):=\{(\rho, a)$ où $\rho$ est une représentation cuspidale irréductible d'un certain groupe linéaire $G L\left(d_{\rho}, E\right)$ (cela définit $d_{\rho}$ ) et $a \in \mathbf{N}$ avec les conditions que l'on décrira ci-dessous et une application

$$
\epsilon_{\pi_{0}}: \operatorname{Jord}\left(\pi_{0}\right) \rightarrow\{ \pm 1\} .
$$

On aura besoin de la définition suivante. Fixons $\rho$ une représentation cuspidale unitaire irréductible d'un groupe linéaire $G L\left(d_{\rho}, E\right)$; cela définit $d_{\rho}$. On considère $G L\left(d_{\rho}, E\right)$ comme un groupe sur $F$ et son $L$-groupe est isomorphe au produit semi-direct:

$$
\left(G L\left(d_{\rho}, \mathbf{C}\right) \times G L\left(d_{\rho}, \mathbf{C}\right)\right) \ltimes G a l(E / F),
$$

où l'élément non trivial, $\sigma$ de $\operatorname{Gal}(E / F)$ agit par:

$$
\sigma\left(g, g^{\prime}\right)=\left({ }^{t} g^{\prime-1},{ }^{t} g^{-1}\right) .
$$

Pour $\eta= \pm 1$, on note $R_{\eta}$ la représentation de ce $L$-groupe dans $\operatorname{End}\left(\mathbf{C}^{d_{\rho}}\right)$ défini par:

$$
\forall u \in \operatorname{End}\left(\mathbf{C}^{d_{\rho}}\right), \quad \forall g, g^{\prime} \in G L\left(d_{\rho}, \mathbf{C}\right),\left(g, g^{\prime}, 1\right) .(u)=g u^{t} g^{\prime}
$$

et

$$
(1,1, \sigma)(u)=\eta^{t} u
$$

Pour $\eta=(-1)^{n}, R_{\eta}$ est une représentation qui intervient naturellement dans la représentation adjointe du $L$-groupe du Levi $G L\left(d_{\rho}, E\right) \times U(n, E / F)$ dans le radical unipotent de son parabolique.

La fonction $L, L\left(\rho, R_{+} \oplus R_{-}, s\right)$ a un pôle en $s=0$ exactement quand $\rho \simeq \sigma \rho^{*}$ et, si cet isomorphisme est réalisé, on note $\lambda_{\rho}$ la valeur de $\eta$ pour laquelle $L\left(\rho, R_{\eta}, s\right)$ a un pôle en $s=0$.

On définit alors $\operatorname{Jord}\left(\pi_{0}\right)$ de la façon suivante: $(\rho, a) \in \operatorname{Jord}\left(\pi_{0}\right)$ si et seulement si $\rho^{*} \simeq{ }^{\sigma} \rho$ où $\sigma$ est l'élément non trivial du groupe de Galois de $E / F$; et les 2 conditions:

$a$ est pair (resp. impair) si $\lambda_{\rho}=(-1)^{n}\left(\operatorname{resp} \lambda_{\rho}=(-1)^{n-1}\right.$;

en notant $S D(\rho, a)$ la série discrète de $G L\left(a d_{\rho}, E\right)$ basée sur $\rho$, l'induite $S D(\rho, a) \times \pi_{0}$ du groupe $U\left(n+2 a d_{\rho}, E / F\right)$ est irréductible.

Avec une telle définition, il n'est pas du tout clair ni que $\operatorname{Jord}\left(\pi_{0}\right)$ soit non vide ni que $\operatorname{Jord}\left(\pi_{0}\right)$ soit fini mais une conjecture naturelle est que cet ensemble soit fini et vérifie:

$$
\sum_{(\rho, a) \in \operatorname{Jord}\left(\pi_{0}\right)} a d_{\rho}=n .
$$

Le problème se pose pour les cuspidales, si on a une telle relation pour les cuspidales, alors on l'a pour les séries discrètes. Comme nous n'avons pas besoin de cette hypothèse nous ne la faisons pas. On prendra des hypothèses telles que pour $\rho$ fixé, 
l'ensemble $\operatorname{Jord}_{\rho}\left(\pi_{0}\right):=\left\{a \in \mathbf{N}\right.$ tel que $\left.(\rho, a) \in \operatorname{Jord}\left(\pi_{0}\right)\right\}$ est nécessairement fini. Cela nous suffit.

L'application $\epsilon_{\pi_{0}}$ n'a vraisemblablement pas une définition complètement canonique. Là encore le problème est pour les cuspidales. Comme pour les groupes symplectiques et orthogonaux impairs, nous n'avons pas besoin, pour classifier les séries discrètes de toute l'application $\epsilon_{\pi_{0}}$ mais seulement de ses "différences", c'est-à-dire des valeurs:

$$
\epsilon_{\pi_{0}}(\rho, a) \epsilon_{\pi_{0}}\left(\rho, a^{\prime}\right)^{-1},
$$

pour $(\rho, a),\left(\rho, a^{\prime}\right) \in \operatorname{Jord}\left(\pi_{0}\right)$ (le même $\rho$ ) en autorisant $a^{\prime}=0$ si $a$ est pair. Pour définir ces nombres, on fixe $(\rho, a) \in \operatorname{Jord}\left(\pi_{0}\right)$ et on note $a_{-}$le plus grand entier $(\geq 0)$ tel que $\left(\rho, a_{-}\right) \in \operatorname{Jord}\left(\pi_{0}\right)$ éventuellement $a_{-}=0$ si $a$ est pair et $a_{-}<a$. On pose alors:

$$
\epsilon_{\pi_{0}}(\rho, a) \epsilon_{\pi_{0}}\left(\rho, a_{-}\right)^{-1}=1,
$$

si et seulement si l'indice de Witt de $U(n, E / F)$ est $\geq d_{\rho}\left(a+a_{-}\right) / 2$ et il existe une représentation $\pi_{0}^{\prime}$ de $U\left(n-d_{\rho}\left(a+a_{-}\right), E / F\right)$ et une inclusion:

$$
\pi_{0} \hookrightarrow S D\left(\rho,(a-1) / 2,-\left(a_{-}-1\right) / 2\right) \times \pi_{0}^{\prime},
$$

où $S D\left(\rho,(a-1) / 2,-\left(a_{-}-1\right) / 2\right)$ est l'unique sous-module irréductible de l'induite de $G L\left(d_{\rho}\left(a+a_{-}\right) / 2, E\right)$ :

$$
\times_{j \in\left[(a-1) / 2,-\left(a_{-}-1\right) / 2\right]} \rho||_{E}^{j} .
$$

A.2.2 Mesure de Plancherel. Evidemment, je ne sais pas, sans hypothèse calculer la mesure de Plancherel associée à des induites de séries discrètes, même à des induites de cuspidales dans le cas d'un parabolique maximal. C'est pourtant cela dont on a besoin pour classifier les séries discrètes. On prend donc des hypothèses pour pouvoir mener à bien la classification et on justifiera ci-dessous ces hypothèses.

L'hypothèse de base est la suivante: soit $\rho$ une représentation cuspidale unitaire irréductible d'un groupe linéaire $G L\left(d_{\rho}, E\right)$ (cela définit $d_{\rho}$ ) et soit $\pi_{0}$ une représentation cuspidale irréductible d'un groupe unitaire $U(n, E / F)$. On considère l'induite pour le groupe $U\left(n+2 d_{\rho}, E / F\right)$ :

$$
\rho||_{E}^{S} \times \pi_{0} .
$$

On sait (par Harish-Chandra) que cette induite ne peut être réductible en un point $s \in \mathbf{R}$ que si $\rho \simeq{ }^{\sigma} \rho^{*}$ et on prend comme hypothèse, qu'avec cette propriété de $\rho$ elle est réductible en $s=s_{0}$ si et seulement si:

$$
s_{0}= \pm\left(a_{\rho}+1\right) / 2 \text {, }
$$

où $a_{\rho}=\sup _{a \in \mathbf{N}}\left\{a \mid(\rho, a) \in \operatorname{Jord}\left(\pi_{0}\right)\right\}$ si cet ensemble est non vide et fini, sinon $a_{\rho}=-1$ si $\lambda_{\rho}=(-1)^{n-1}$ et $a_{\rho}=0$ si $\lambda_{\rho}=(-1)^{n}$.

Lemme: Avec ces hypothèses sur les points de réductibilité et en supposant $\pi_{0}$ cuspidal, si $(\rho, a) \in \operatorname{Jord}\left(\pi_{0}\right)$ avec $a \geq 3$, alors $(\rho, a-2) \in \operatorname{Jord}\left(\pi_{0}\right)$. En outre $\operatorname{Jord}_{\rho}\left(\pi_{0}\right)$ est fini.

C'est un calcul de module de Jacquet: supposons que $(\rho, a) \notin \operatorname{Jord}\left(\pi_{0}\right)$ (avec $a$ un entier de la bonne parité) et montrons que $(\rho, a+2) \notin \operatorname{Jord}\left(\pi_{0}\right)$ : en effet, on 
note $\pi_{\epsilon}$ pour $\epsilon= \pm 1$ les deux sous-modules de $S D(\rho, a) \times \pi_{0}$ et on regarde les induites suivantes:

$$
\begin{gathered}
S D(\rho, a+2) \times \pi_{0}, \\
\rho||^{(a+1) / 2} \times\left.\rho\right|^{(a+1) / 2} \times S D(\rho, a) \times \pi_{0} ; \\
\rho||^{(a+1) / 2} \times\left.\rho\right|^{(a+1) / 2} \times \pi_{\epsilon} .
\end{gathered}
$$

On calcule les modules de Jacquet de ces induites relativement au parabolique de Levi $G L\left(2 d_{\rho}, E\right) \times U\left(n+2 a d_{\rho}, E / F\right)$ et plus précisément la projection de ce module de Jacquet en ne gardant que les termes dont le support cuspidal pour le facteur $G L\left(2 d_{\rho}, E\right)$ est celui de l'induite irréductible $\rho\left\|^{(a+1) / 2} \times \rho\right\|^{(a+1) / 2}$. Pour les 2 premières induites, cette projection est exactement la représentation:

$$
\rho||^{(a+1) / 2} \times\left.\rho\right|^{(a+1) / 2} \otimes S D(\rho, a) \times \pi_{0}
$$

tandis que pour la troisième avec $\epsilon= \pm 1$, on trouve:

$$
\rho||^{(a+1) / 2} \times \rho||^{(a+1) / 2} \otimes \pi_{\epsilon} .
$$

Cela prouve que la première induite n'est pas irréductible. On vient donc de montrer que si $\rho \times \pi_{0}$ est réductible, alors pour tout $a$ impair $S D(\rho, a) \times \pi_{0}$ est aussi réductible et donc $(\rho, a) \notin \operatorname{Jord}\left(\pi_{0}\right)$ pour tout $a$ impair.

Un calcul de module de Jacquet très facile, montre que $S D(\rho, 2) \times \pi_{0}$ est irréductible si et seulement si $\rho||^{1 / 2} \times \pi_{0}$ est irréductible. En particulier si $\left.\rho\right|^{1 / 2} \times$ $\pi_{0}$ est réductible, alors $(\rho, a) \notin \operatorname{Jord}\left(\pi_{0}\right)$ pour tout $a$ pair.

On peut maintenant conclure: supposons d'abord que le seul point de réductibilité soit en 0. Par la conjecture, on a admis qu'il n'y a pas de pôle à la fonction $L$ (pour $\rho$ et $R_{(-1)^{n}}$ ) en $s=0$; par définition, si $(\rho, a) \in \operatorname{Jord}\left(\pi_{0}\right)$, a doit être impair et on a alors vu ci-dessus que pour tout $a$ impair $(\rho, a) \notin \operatorname{Jord}\left(\pi_{0}\right)$. On a donc montré que si le point de réductibilité est en 0 , alors $\operatorname{Jord}_{\rho}\left(\pi_{0}\right)$ est vide. Un raisonnement analogue montre que si les points de réductibilité sont en $\pm 1 / 2$, alors aussi $\operatorname{Jord}_{\rho}\left(\pi_{0}\right)$ est vide.

On a donc en particulier vérifié, que la conjecture entraîne la finitude de $\operatorname{Jord}_{\rho}\left(\pi_{0}\right)$. Supposons que cet ensemble soit fini et non vide et soit $\left(\rho, a_{\rho}\right)$ son élément maximum. Par définition si $(\rho, a) \in \operatorname{Jord}\left(\pi_{0}\right)$ alors $a$ a la même parité que $a_{\rho}$; supposons donc qu'il existe $a<a_{\rho}$ de même parité et tel que $(\rho, a) \notin \operatorname{Jord}\left(\pi_{0}\right)$; d'après ce que l'on vient de voir, cela entraînerait aussi que $\left(\rho, a_{\rho}\right) \notin \operatorname{Jord}\left(\pi_{0}\right)$ ce qui est absurde.

Remarque: Ici $\pi_{0}$ est une représentation cuspidale irréductible de $U(n, E / F)$ isomorphe à $\sigma \pi_{0}^{*}$. L'hypothèse faite sur la réductibilité des induites de cuspidales est équivalente à dire que le composé des opérateurs d'entrelacement standard (sous l'hypothèse $\rho \simeq{ }^{\sigma} \rho^{*}$ ):

$$
\rho||_{E}^{s} \times \pi_{0} \rightarrow \rho||_{E}^{-s} \times \pi_{0} \rightarrow \rho||_{E}^{s} \times \pi_{0}
$$


est à une fonction holomorphe inversible près (au voisinage de l'axe réel) égale à

$$
\begin{gathered}
\times(\rho, a) \in \operatorname{Jord}\left(\pi_{0}\right) L(\rho \times \rho, s-(a-1) / 2) L(\rho \times \rho,-s-(a-1) / 2) \times \\
L(\rho \times \rho, s+(a+1) / 2)^{-1} L(\rho \times \rho,-s+(a+1) / 2)^{-1} \times \\
L\left(\rho, R_{n}, 2 s\right) L\left(\rho, R_{n},-2 s\right) L\left(\rho, R_{n}, 2 s+1\right)^{-1} L\left(\rho, R_{n},-2 s+1\right)^{-1},
\end{gathered}
$$

où $R_{n}$ est la représentation $R_{(-1)^{n}}$.

Pour montrer notre assertion, il suffit de calculer les zéros et les pôles de cette fonction puisque d'après Harish-Chandra, le composé de ces opérateurs est une fonction méromorphe de $s$ ayant au plus un pôle en $s=0$ (il est alors double) et ayant des zéros simples en tout point de réductibilité autre que $s=0$. En outre, toujours d'après Harish-Chandra, le pôle existe si et seulement si l'induite est irréductible en $s=0$.

Si $\operatorname{Jord}\left(\pi_{0}\right)$ ne contient aucun élément du type $(\rho, a)$ avec le $\rho$ fixé, alors seul compte le dernier terme qui a soit un pôle double en $s=0$ et un zéro simple en $s= \pm 1 / 2$, soit est holomorphe inversible en tout point réel, cela dépend précisément du produit $\lambda_{\rho}(-1)^{n}$. Admettons cette formule pour le produit des opérateurs, on peut alors utiliser les résultats d'Harish-Chandra pour trouver les points de réductibilité: si $\lambda_{\rho}(-1)^{n}=1$, alors, on est dans le premier cas, c'est-àdire d'après Harish-Chandra, irréductibilité en $s=0$ et réductibilité en $s= \pm 1 / 2$. Sinon, on a réductibilité en $s=0$ et nulle part ailleurs. C'est bien équivalent à notre assertion sur la réductibilité.

Supposons maintenant que $\operatorname{Jord}\left(\pi_{0}\right)$ contient un élément $(\rho, a)$; on note $a_{\rho}$ l'entier maximal avec cette propriété. La première ligne se simplifie grâce à ce que l'on vient de voir sur $\operatorname{Jord}\left(\pi_{0}\right)$ à savoir que si $(\rho, a) \in \operatorname{Jord}\left(\pi_{0}\right)$ avec $a \geq 3$ alors $(\rho, a-2) \in \operatorname{Jord}\left(\pi_{0}\right)$. On note $a_{\text {min }}$ le plus petit entier tel que $(\rho, a) \in \operatorname{Jord}\left(\pi_{0}\right)$; par hypothèse sur les parités, $a_{\text {min }}=1$ si $\lambda_{\rho} \neq(-1)^{n}$ et 2 sinon. Le produit des opérateurs devient (en négligeant des fonctions sans pôles ni zéros en $s$ réel) et en utilisant la formule explicite pour $\left.L\left(\rho \times \rho, s^{\prime}\right)\right)$ :

$$
\begin{gathered}
L\left(\rho \times \rho, s-\left(a_{\min }-1\right) / 2\right) \times \\
L\left(\rho \times \rho,-s-\left(a_{\min }-1\right) / 2\right) / L\left(\rho \times \rho, s+\left(a_{\rho}+1\right) / 2\right) \times \\
L\left(\rho \times \rho,-s+\left(a_{\rho}+1\right) / 2\right) \times \\
L\left(\rho, R_{n}, 2 s\right) L\left(\rho, R_{n},-2 s\right) / L\left(\rho, R_{n}, 2 s+1\right) L\left(\rho, R_{n},-2 s+1\right) .
\end{gathered}
$$

Si $\lambda_{\rho}=(-1)^{n}$, le pôle en $s=0$ de la deuxième ligne n'est pas compensé par un zéro, d'après Harish-Chandra c'est signe d'irréductibilité en $s=0$, par contre, les zéros de la deuxième ligne (en $s= \pm 1 / 2$ ) sont compensés par des pôles de la première ligne dû au fait que $a_{\min }=2$. Les seuls zéros sont alors en $s= \pm\left(a_{\rho}+1\right) / 2$. C'est équivalent à l'hypothèse que l'on a prise. Dans le cas où $\lambda_{\rho} \neq(-1)^{n}$, la deuxième ligne n'a ni zéro ni pôle et la première ligne à un pôle double en $s=0$ dû au fait que $a_{\min }=1$ et des zéros simples en $s= \pm\left(a_{\rho}+1\right) / 2$. Cela termine la preuve de l'équivalence des 2 assertions.

Evidemment cette formulation pour le produit des opérateurs d'entrelacement vient directement du calcul de la représentation adjointe dans $1 \mathrm{e}^{L}$-groupe. 


\section{A.3 Le cas des groupes orthogonaux pairs}

Ici $G$ est un groupe orthogonal sur un espace, $V$, de dimension paire, $2 n$, déployé ou non. Suivant une vieille idée d'Adams, on pose

$$
{ }^{L} G:=O(2 n, \mathbf{C}) .
$$

On ne fait pas apparaître $W_{F}$ car $O(2 n, \mathbf{C})$ n'a pas d'automorphismes extérieurs non triviaux. On note $\eta_{V}$ le discriminant de $V$ et $\epsilon_{V}$ son invariant de Hasse. On prend les normalisations usuelles de telle sorte que ses nombres ne dépendent que du noyau anisotrope de $V$ et que l'espace anisotrope $V_{0}$ d'invariant de Hasse -1 et de discriminant 1 soit l'espace anisotrope de dimension 4. On s'intéresse aux homomorphismes, $\phi$, de $W_{F}$ dans $O(2 n, \mathbf{C})$ dont le déterminant est le caractère de $W_{F}$ correspondant à $\eta_{V}$. On ne considérera aussi que les caractères du centralisateur de $\phi$ dont la restriction au centre de $O(2 n, \mathbf{C})$ coïncide avec le caractère $\epsilon_{V}$. Ceci pour prendre en compte le caractère éventuellement non déployé du groupe.

La difficulté n'est pas de classifier ces homomorphismes et ces caractères. On ne s'intéresse qu' aux homomorphismes qui se prolongent en un homomorphisme, encore noté $\phi$ de $W_{F} \times S L(2, \mathbf{C})$ dans $O(2 n, \mathbf{C})$ et dont le centralisateur est un groupe fini. Ces homomorphismes, à conjugaison près sont classifiés par les ensembles, $\operatorname{Jord}(\phi)$ (sans multiplicité) de la forme $\{(\rho, a)\}$ où $\rho$ est une représentation irréductible de $W_{F}$ de dimension $d_{\rho}$ (cela définit $d_{\rho}$ ) isomorphe à $\rho^{*}$ et où $a$ est un entier pair si $L\left(\rho, \wedge^{2} \mathbf{C}^{d_{\rho}}, s\right)$ a un pôle en $s=0$ et impair sinon. On a en plus les 2 relations:

$$
\sum_{(\rho, a) \in \operatorname{Jord}(\phi)} a d_{\rho}=2 n ; \quad \prod_{(\rho, a) \in \operatorname{Jord}(\phi)}(\operatorname{det} \rho)^{a}=\eta_{V} .
$$

Dans ces conditions, le groupe des caractères du centralisateur de $\phi$ est isomorphe au groupe des applications:

$$
\epsilon_{\phi}: \operatorname{Jord}(\phi) \rightarrow\{ \pm 1\}
$$

La condition sur la restriction au centre de $O(2 n, \mathbf{C})$ n'est autre que:

$$
\prod_{(\rho, a) \in \operatorname{Jord}(\phi)} \epsilon_{\phi}^{a d_{\rho}}=\epsilon_{V}
$$

Le côté représentation est maintenant facile à expliquer. Soit $\pi_{0}$ une série discrète de $O(2 n, \mathbf{C})$, on suppose pour le moment que $n \neq 0$; on définit $\operatorname{Jord}\left(\pi_{0}\right)$ comme l'ensemble des couples $(\rho, a)$ tel que $\rho$ est une représentation cuspidale unitaire irréductible d'un groupe $G L\left(d_{\rho}, \mathbf{C}\right)$, isomorphe à sa contragrédiente et $a$ est entier pair si $L\left(\rho, \wedge^{2} \mathbf{C}^{d_{\rho}}, s\right)$ a un pôle en $s=0$ et impair sinon et l'induite:

$$
S D(\rho, a) \times \pi_{0}
$$

est irréductible. L'hypothèse sur la dimension est que:

$$
\sum_{(\rho, a) \in \operatorname{Jord}\left(\pi_{0}\right)} a d_{\rho}=2 n
$$

mais nous n'en avons pas besoin. 
Quant à l'hypothèse sur la réductibilité des induites de cuspidales, c'est la généralisation immédiate de celles concernant les groupes symplectiques: soit $\rho$ et $\pi_{0}$ comme ci-dessus, alors l'induite $\left.\rho\right|^{s} \times \pi_{0}$ pour $s \in \mathbf{R}$ est réductible si et seulement si:

$$
s= \pm\left(a_{\rho}+1\right) / 2 \text {, }
$$

où $a_{\rho}$ est le plus grand entier, $a$, tel que $(\rho, a) \in \operatorname{Jord}\left(\pi_{0}\right)$ si cet ensemble est non vide et $a_{\rho}=1$ (resp. 2) si cet ensemble est vide alors que $L\left(\rho, \wedge^{2} \mathbf{C}^{d_{\rho}}, s\right)$ n'a pas de pôle en $s=0$, (resp. en a un).

Ce qui nous intéresse est de vérifier:

Remarque: soit $\pi_{0}$ une représentation cuspidale irréductible de $O(2 n, F)$ isomorphe à sa contragrédiente et soit $\rho$ comme ci-dessus. L'hypothèse sur les points de réductibilité des induites $\left.\rho\right|^{S} \times \pi_{0}$ est équivalente à ce que le composé des opérateurs d'entrelacement standard:

$$
\rho||^{s} \times \pi_{0} \rightarrow \rho||^{-s} \times \pi_{0} \rightarrow \rho||^{s} \times \pi_{0}
$$

est à une fonction holomorphe inversible au voisinage de $\mathbf{R}$ près, égal à:

$$
\begin{gathered}
\prod_{(\rho, a) \in \operatorname{Jord}\left(\pi_{0}\right)} L(\rho \times \rho, s-(a-1) / 2) \times \\
L(\rho \times \rho,-s-(a-1) / 2) / L(\rho \times \rho, s+(a+1) / 2) L(\rho \times \rho,-s+(a+1) / 2) \times \\
L\left(\rho, \wedge^{2}, \mathbf{C}^{d_{\rho}}, 2 s\right) L\left(\rho, \wedge^{2}, \mathbf{C}^{d_{\rho}},-2 s\right) / L\left(\rho, \wedge^{2} \mathbf{C}^{d_{\rho}}, 2 s+1\right) \times \\
L\left(\rho, \wedge^{2} \mathbf{C}^{d_{\rho}},-2 s+1\right) .
\end{gathered}
$$

On suppose d'abord que $n \neq 0$. Dans ce cas, la démonstration se fait comme dans le cas des groupes unitaires pour la simple raison qu'en notant $\pi_{0}^{\prime} n$ 'importe quel sous- $S O(2 n)$-représentation de $\pi_{0}$, on a les équivalence:

$$
S D(\rho, a) \times \pi_{0} \text { est irréductible } \Leftrightarrow S D(\rho, a) \times \pi_{0}^{\prime} \text { est irréductible }
$$

et la même équivalence pour l'irréductibilité de $\rho||^{s} \times \pi_{0}$ et $\rho||^{s} \times \pi_{0}^{\prime}$ en un point $s=s_{0}$ fixé. Cela vient de l'hypothèse $n \neq 0$.

Par contre, nous avons évidemment besoin d'accepter aussi l'hypothèse $n=0$, auquel cas $\pi_{0}$ disparaît. Quand on travaille avec un groupe orthogonal pair (ici $O\left(2 d_{\rho}\right)$, nécessairement déployé) les 2 paraboliques du groupe spécial orthogonal de Levi isomorphe à $G L\left(d_{\rho}\right)$ sont conjugués. On peut donc sans ambiguité définir:

$$
\text { ind }^{O\left(2 d_{\rho}\right)} \rho||^{s} \text {. }
$$

La généralisation de la conjecture à ce cas devient:

l'induite ci-dessus est réductible en un point $s=s_{0}$ si et seulement si $\rho \simeq \rho^{*}$ et $s=0$ si $L\left(\rho, \wedge^{2} \mathbf{C}^{d_{\rho}}, s\right)$ n'a pas de pôle en $s=0$ et en $s= \pm 1 / 2$ si au contraire il y a un pôle.

cette assertion se démontre à l'aide des résultats de Shahidi [ $\left.\mathrm{S}_{2}\right]$ et d'HarishChandra $[\mathrm{W}]$. Le premier cas à considérer est le cas où $d_{\rho}$ est impair. Dans ce cas, 
on note $P_{+}$et $P_{-}$les 2 paraboliques de $S O\left(2 d_{\rho}\right)$ de Levi isomorphe à $G L\left(d_{\rho}\right)$. Les Levi de ces paraboliques sont conjugués dans $S O\left(2 d_{\rho}\right)$ alors que la représentation:

$$
\operatorname{ind}_{P_{\eta}}^{S O\left(2 d_{\rho}\right)} \rho||^{S}
$$

pour $\eta= \pm 1$ est irréductible pour tout $s \in \mathbf{R}$ faute d'entrelacement. Ces induites sont donc isomorphes. En $s=0$ et sous l'hypothèse que $\rho \simeq \rho^{*}$, les éléments de $O(2 n)-S O(2 n)$ laissent stable la classe d'isomorphie de cette représentation. La théorie de Mackey dit alors que l'induite à $O(2 n)$ est elle-même réductible ce qui est l'assertion que nous cherchions puis que $L\left(\rho, \wedge^{2} \mathbf{C}^{d_{\rho}}, s\right)$ n'a pas de pôle en $s=0, d_{\rho}$ étant impair ([S2] 7.6).

Supposons maintenant que $d_{\rho}$ est pair. Les résultats de Shahidi ([S2] 7.6) assurent que l'induite:

$$
\operatorname{ind}_{P_{\eta}}^{S O(2 n)} \rho||^{S}
$$

est irréductible en $s=0$ si et seulement si $L\left(\rho, \wedge^{2} \mathbf{C}^{d_{\rho}}, s\right)$ a un pôle en $s=0$. Et avec $\left(\left[\mathrm{S}_{2}\right] \mathrm{8.1}\right)$, on sait que s'il y a un pôle alors les points de réductibilité sont en $s= \pm 1 / 2$. Pour nous la seule chose à démontrer et donc que:

$$
\operatorname{ind}_{P_{\eta}}^{O(2 n)} \rho||^{s}
$$

est irréductible si et seulement si cela est vrai pour l'induite à $S O(2 n)$. Le seulement si est évidemment trivial et montrons le si. Soit $x$ un élément de $O(2 n)$ conjugant $P_{+}$en $P_{-}$. On a:

$$
x\left(\operatorname{ind}_{P_{+}}^{S O(2 n)} \rho||^{s}\right) \simeq i n d_{P_{-}}^{S O(2 n)} \rho||^{s} .
$$

Or aucun sous-quotient de $i n d_{P_{+}}^{S O(2 n)} \rho||^{S}$ n'est isomorphe à un sous-quotient de $i_{n d}^{S O(2 n)} \rho||^{s}$; cela se voit en regardant les modules de Jacquet qui sont complètement disjoints. Notre assertion résulte alors de la théorie de Mackey.

\section{Bibliographie}

[A1] Arthur, J.: Intertwining Operators and Residues 1: weighted Characters. J. Funct. Analysis 84, 19-84 (1989)

[A2] Arthur, J.: Unipotent automorphic representations: global motivations in Automorphic forms, Shimura varieties and L-functions, vol 1 ed Clozel and Milne, Birkhauser, PM 10, 1990, pp. 1-75

[J] Jantzen, C.: On square integrable representations of classical p-adic groups. Can. J. Math. 52, 539-581 (2000)

[M1] Møglin, C.: Normalisation des opérateurs d'entrelacement et réductibilité des induites de cuspidales; le cas des groupes classiques p-adiques. Ann. Math. 151, 817-847 (2000)

[M2] Mœglin, C.: Représentations quadratiques unipotentes des groupes classiques padiques. Duke Math. J. 84, 267-332 (1996)

[Mu1] Muić, G.: Some results on square integrable representations irreducibility of standard representations. Int. Math. Res. Notices 14, 705-726 (1998)

[Mu 2] Muić, G.: On generic irreducible representations of $\operatorname{Sp}(n, F)$ and $S O(2 n+1, F)$. Glasnik Mathematicki 33, 19-31 (1998) 
[M-T] Mœglin, C., Tadić, M.: Construction of discrete series for classical p-adic groups. (prépublication 1999)

[M-W] Mœglin, C., Waldspurger, J.-L.: Le spectre résiduel de GL(n). Ann. de l'ENS 22, 605-674 (1989)

[S1] Shahidi, F.: On certain L-functions. Am. J. Math. 103, 297-356 (1981)

[S2] Shahidi, F.: A proof of Langlands conjecture on Plancherel measures: complementary series for p-adic groups. Ann. Math. 132, 273-330 (1990)

[T1] Tadić, M.: On regular square integrable representations of $p$-adic groups. Am. J. Math. 120, 159-210 (1998)

[T2] Tadić, M.: Square integrable representations of classical $p$-adic groups corresponding to segments. (prépublication)

[T3] Tadić, M.: A family of square integrable representations of classical $p$-adic groups in the case of general half-integral reducibilities. (prépublication)

[T4] Tadić, M.: On square integrable representations of classical $p$-adic groups. (prépublication)

[W] Waldspurger, J.-L.: La formule de Plancherel pour les groupes p-adiques d'après Harish-Chandra. (prépublication)

[Z] Zelevinski, A.: Induced representations of reductive p-adic groups II. On irreductible representations of GL(n). Ann. Scient. Ec. Norm. Sup. 13, 165-210 (1980) 